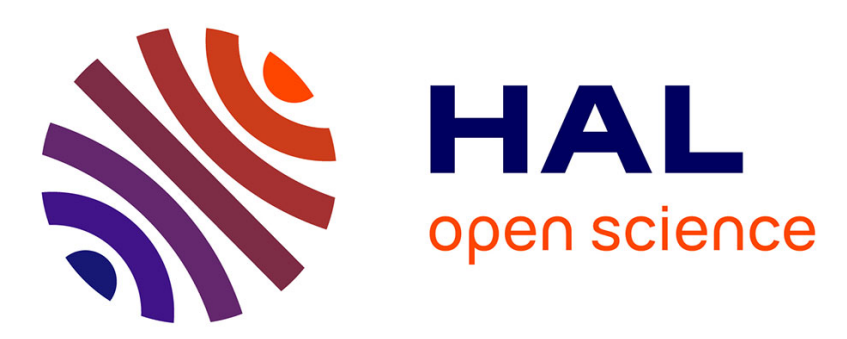

\title{
Analysis of a stabilized penalty-free Nitsche method for the Brinkman, Stokes, and Darcy problems
}

Laura Blank, Alfonso Caiazzo, Franz Chouly, Alexei Lozinski, Joaquin Mura

\section{To cite this version:}

Laura Blank, Alfonso Caiazzo, Franz Chouly, Alexei Lozinski, Joaquin Mura. Analysis of a stabilized penalty-free Nitsche method for the Brinkman, Stokes, and Darcy problems. ESAIM: Mathematical Modelling and Numerical Analysis, 2018, 52 (6), pp.2149-2185. 10.1051/m2an/2018063 . hal-01725387

\section{HAL Id: hal-01725387 \\ https://hal.science/hal-01725387}

Submitted on 7 Mar 2018

HAL is a multi-disciplinary open access archive for the deposit and dissemination of scientific research documents, whether they are published or not. The documents may come from teaching and research institutions in France or abroad, or from public or private research centers.
L'archive ouverte pluridisciplinaire HAL, est destinée au dépôt et à la diffusion de documents scientifiques de niveau recherche, publiés ou non, émanant des établissements d'enseignement et de recherche français ou étrangers, des laboratoires publics ou privés. 


\title{
ANALYSIS OF A STABILIZED PENALTY-FREE NITSCHE METHOD FOR THE BRINKMAN, STOKES, AND DARCY PROBLEMS
}

\author{
Laura Blank ${ }^{1}$, Alfonso Caiazzo ${ }^{1}$, Franz Chouly ${ }^{2}$, Alexei Lozinski ${ }^{2}$ And \\ JOAQUIN MURA ${ }^{3}$
}

\begin{abstract}
In this paper we study the Brinkman model as a unified framework to allow the transition between the Darcy and the Stokes problems. We propose an unconditionally stable low-order finite element approach, which is robust with respect to the whole range of physical parameters, and is based on the combination of stabilized equal-order finite elements with a non-symmetric penalty-free Nitsche method for the weak imposition of essential boundary conditions. In particular, we study the properties of the penalty-free Nitsche formulation for the Brinkman setting, extending a recently reported analysis for the case of incompressible elasticity (T. Boiveau 8 E. Burman. IMA J. Numer. Anal. 36 (2016), no.2, 770-795). Focusing on the two-dimensional case, we obtain optimal a priori error estimates in a mesh-dependent norm, which, converging to natural norms in the cases of Stokes or Darcy flows, allows to extend the results also to these limits. Moreover, we show that, in order to obtain robust estimates also in the Darcy limit, the formulation shall be equipped with a Grad-Div stabilization and an additional stabilization to control the discontinuities of the normal velocity along the boundary. The conclusions of the analysis are supported by numerical simulations.
\end{abstract}

1991 Mathematics Subject Classification. 65N30, 65N12, 65N15.

\section{INTRODUCTION}

The Brinkman problem 10], originally proposed as an alternative model approach for the flow in porous media, is obtained as a modification of the Darcy model by equipping Darcy's law with a resistance term proportional to the fluid viscous stresses, targeting on a better handling of high permeability regions.

In order to introduce the model problem of interest, let us consider a connected domain $\boldsymbol{\Omega} \subset \mathbb{R}^{n}, n=2,3$, with boundary $\Gamma:=\partial \boldsymbol{\Omega}$, and let us denote by $\boldsymbol{n}$ the outer unit normal vector on $\Gamma$. Our model problem is described by the following system of partial differential equations

$$
\begin{aligned}
-\nabla \cdot\left(\mu_{\mathrm{eff}} \nabla \boldsymbol{u}\right)+\sigma \boldsymbol{u}+\nabla p & =\boldsymbol{f}, & & \text { in } \boldsymbol{\Omega}, \\
\nabla \cdot \boldsymbol{u} & =g, & & \text { in } \boldsymbol{\Omega},
\end{aligned}
$$

Keywords and phrases: Brinkman problem, penalty-free Nitsche method, weak boundary conditions, stabilized finite elements

1 Weierstraß-Institut für Angewandte Analysis und Stochastik, Mohrenstr. 39, 10117 Berlin, Germany.

e-mail: laura.blank@wias-berlin.de \& alfonso.caiazzo@wias-berlin.de

2 Laboratoire de Mathématiques de Besançon UMR CNRS 6623, Université Bourgogne Franche Comté, 16 route de Gray, 25030

Besançon Cedex, France. e-mail: franz.chouly@univ-fcomte.fr \& alexei.lozinski@univ-fcomte.fr

3 Biomedical Imaging Center, School of Engineering and School of Medicine, Pontificia Universidad Católica de Chile, Av. Vicuña Mackenna 4860, 7820436 Santiago, Chile. e-mail: jamura@uc.cl 
where $\boldsymbol{u}: \boldsymbol{\Omega} \rightarrow \mathbb{R}^{n}$ represents the fluid velocity field, $p: \boldsymbol{\Omega} \rightarrow \mathbb{R}$ is the fluid pressure and $\boldsymbol{f}: \boldsymbol{\Omega} \rightarrow \mathbb{R}{ }^{n}, g: \boldsymbol{\Omega} \rightarrow \mathbb{R}$ are given data. In 1.1 , the parameter $\mu_{\text {eff }}$ is called effective viscosity, while $\sigma$ is given by the ratio between the fluid viscosity and the permeability of the porous medium. Depending on the values of the aforementioned parameters, the system 1.1 describes a whole range of problems between the Stokes $(\sigma=0)$ and the Darcy $\left(\mu_{\mathrm{eff}}=0\right)$ models.

However, this transition does not depend continuously on the physical parameters. In particular, the standard boundary condition for $\mu_{\mathrm{eff}}>0$ is

$$
\boldsymbol{u}=\mathbf{0}, \quad \text { on } \Gamma,
$$

(essential boundary condition on the velocity $\boldsymbol{u}$ ), whereas for $\mu_{\mathrm{eff}}=0$, it has to be replaced by the condition

$$
\boldsymbol{u} \cdot \mathbf{n}=0, \quad \text { on } \Gamma
$$

which is appropriate for the Darcy problem. Likewise, when focusing on the weak counterpart of 1.1), one has to consider different natural functional settings for the Stokes/Brinkman $\left(\mu_{\mathrm{eff}}>0\right)$ and Darcy $\left(\mu_{\mathrm{eff}}=0\right)$ problems.

These aspects affect also the discrete formulation of (1.1) and the strategies for its numerical solution. In the context of finite element methods, the different regularity properties of the limit problems (Stokes and Darcy) are reflected in the choice of the finite element spaces used for the velocity and the pressure: stable and efficient elements for the Stokes problem might not provide accurate or stable approximations in the Darcy case, and vice versa (see, e.g., $9,12,27]$ ). Moreover, the discrepancy between the boundary conditions in the limit cases at the continuous level implies that imposing essential boundary conditions on the velocity space does not allow a smooth, parameter-dependent transition between $(1.2)$ and $\sqrt{1.3}$, in particular at the discrete level.

Our work is motivated by the solution of direct and inverse problems in clinical applications involving flows in porous media. Hence, the numerical method shall be robust with respect to different flow regimes, in order to handle unknown physical parameters, and, at the same time, require relatively low computational cost, allowing for the numerical solution in a reasonable time.

One strategy to achieve a common discretization for both, the Stokes and the Darcy problems, which will be adopted in this paper, consists in using finite element pairs suited for both cases, possibly including stabilization terms. Among the different possibilities, we focus on equal-order (linear) finite elements, combined with a Galerkin Least Squares (GLS) and a Grad-Div stabilization, that guarantee stability for the pressure and control on the divergence of the velocity. This setting, with a particular focus on the Brinkman, Stokes and Darcy problems, has been deeply analyzed, e.g., in [3], considering different choices for the scaling of the stabilization terms. Other options, which have been proposed in the literature, are based on $\mathbb{P}_{1} / \mathbb{P}_{0}$ (stabilized) finite elements (analyzed in 12 for the Stokes-Darcy coupling and discussed in 22 for the Brinkman problem), Taylor-Hood, MINI, and $\mathbb{P}_{k} / \mathbb{P}_{k}$ (stabilized) elements 21,26 , as well as $\left.\mathbb{P}_{k} / \mathbb{P}_{k-1}^{\text {disc }} 15\right]$.

In order to tackle the issue of the need of different boundary conditions depending on the (Stokes or Darcy) regime, we focus on the weak imposition of essential boundary conditions via a Nitsche method. This approach, originally introduced in [29], has been extended, applied, and analyzed in several contexts, including coupled Stokes-Darcy problems (see, e.g., 12 15] among others), and the general Brinkman problem (see, e.g., 21 22 26]), demonstrating that it is able to yield a robust transition between the two different flow regimes. In its pioneer version 29], the Nitsche approach was formulated as a consistent symmetric penalty method, for which stability was guaranteed choosing the penalty parameter sufficiently large. This assumption was relaxed considering a non-symmetric version proposed in [19], for which stability was proven for any strictly positive value of the penalty parameter.

We investigate the so-called non-symmetric penalty-free Nitsche method, i.e., assessing the stability of the approach even without the presence of a penalty parameter. In this case, the method can be interpreted as a Lagrange multiplier method [31, where for the Brinkman problem the normal fluxes at the boundary and the 
pressure play the role of Lagrange multipliers. The stability of the Nitsche method without penalty was first shown in 11] for convection-diffusion problems, and more recently extended to compressible and incompressible elasticity [5] and to domain decomposition problems with discontinuous material parameters [4].

The unconditional applicability in presence of variable physical parameters is our main motivation for addressing and investigating the properties of the penalty-free Nitsche method for the Brinkman problem. In this case, the main challenges are related to the fact that stability has to be shown for the pressure (in the case of equal-order finite elements) and the velocity at the boundary. For the latter, it is important to observe that due to the differences in the limit problems (e.g., in the boundary conditions $(1.2)$ and $(1.3)$ ), the natural norms to be controlled depend on the physical range.

Our main result concerns the stability, the robustness, and the optimal convergence in a natural norm of the formulation obtained by combining a penalty-free Nitsche method and a stabilized equal-order finite element method. We show that the proposed finite element method is inf-sup stable in the whole range of physical parameters, including the limit values $\mu_{\mathrm{eff}}=0$ or $\sigma=0$. Moreover, our analysis shows that the inf-sup constant does neither depend on $\mu_{\text {eff }}$ nor on $\sigma$, but only on the regularity properties of the mesh and on the stabilization parameters. These results thus extend available estimates recently provided in [21,26] using a similar discrete setting (stabilized finite elements), where the symmetric Nitsche method was analyzed focusing on an adimensional version of (1.1) which does not allow to control the divergence of the velocity and excludes the case $\sigma=0$.

To establish the stability of the Nitsche method, we follow a path inspired by the analysis in [5] for the incompressible elasticity, but proposing a simpler argument. As next, we discuss the stability estimate in the Darcy limit $\mu_{\text {eff }}=0$ (or in the more general case $\frac{\mu_{\text {eff }}}{\sigma} \rightarrow 0$ ), in which only the control on the boundary normal velocity is required. We show that, focusing on the case of two-dimensional polygonal boundaries, an additional stabilization to control the discontinuities of the normal velocity along the boundary is required. To tackle this issue, we introduce a corner stabilization, which penalizes the jump of the normal velocity solely on the corners of the discrete domain and allows to obtain the aforementioned robust stability estimates and optimal a priori error estimates in a mesh-dependent norm.

The paper is organized as follows. In Section 2 we introduce the problem setting, the finite element formulation, and enunciate the main stability and convergence results. Section 3 is dedicated to the technical proofs, while numerical experiments are presented in Section 4. Section 5 draws the conclusive remarks.

\section{A Penalty-free Nitsche Method for the Brinkman Problem}

The purpose of this section is to introduce the stabilized finite element method for the Brinkman problem, the penalty-free Nitsche method for imposing essential boundary conditions, and to state the related stability and convergence results.

\subsection{The weak formulation}

In what follows, we will assume to deal with a two-dimensional domain $\Omega \subset \mathbb{R}^{2}$ (i.e., setting $n=2$ ) with polygonal boundary $\Gamma$. In this setting, let us consider the Sobolev spaces (see, e.g., 1,20$]$ ):

$$
\begin{aligned}
\boldsymbol{H}_{0}^{1}(\boldsymbol{\Omega}) & :=\left\{\boldsymbol{v} \in \boldsymbol{H}^{1}(\boldsymbol{\Omega}):\left.\boldsymbol{v}\right|_{\Gamma}=\mathbf{0}\right\}, \\
\boldsymbol{H}_{\mathrm{div}, 0}(\boldsymbol{\Omega}) & :=\left\{\boldsymbol{v} \in \boldsymbol{L}^{2}(\boldsymbol{\Omega}): \nabla \cdot \boldsymbol{v} \in L^{2}(\boldsymbol{\Omega}),\left.(\boldsymbol{v} \cdot \boldsymbol{n})\right|_{\Gamma}=0\right\}, \\
L_{0}^{2}(\boldsymbol{\Omega}) & :=\left\{q \in L^{2}(\boldsymbol{\Omega}): \int_{\boldsymbol{\Omega}} q=0\right\} .
\end{aligned}
$$

We will denote by curved brackets $(\cdot, \cdot)_{\mathbf{A}}$ the $L^{2}$-scalar product on $\mathbf{A} \subseteq \boldsymbol{\Omega}$, while $\langle\cdot, \cdot\rangle_{E}$, will be used for integrals evaluated on the boundary, i.e., for any $E \subseteq \Gamma$. For the ease of notation, the subscripts $\Omega$ and $\Gamma$ will be omitted, simply denoting with $(\cdot, \cdot)$ and $\langle\cdot, \cdot\rangle$ the scalar products in $L^{2}(\boldsymbol{\Omega})$ and $L^{2}(\Gamma)$, respectively. Furthermore, a bold faced letter will indicate the $n$-th Cartesian power, e.g., $\boldsymbol{H}^{1}(\mathbb{R})=\left[H^{1}(\mathbb{R})\right]^{n}$. Finally, we will denote with $\|\cdot\|_{0}$ 
the norm on $L^{2}(\boldsymbol{\Omega})$, and with $\|\cdot\|_{k}^{2}$ and $|\cdot|_{k}^{2}$ the norm and semi-norm, respectively, on the Sobolev space $H^{k}(\boldsymbol{\Omega})$ (see, e.g., [6, Def. 1.3.1, 1.3.7]).

With the above notations, we now introduce the bilinear forms

$$
\begin{aligned}
A[(\boldsymbol{u}, p) ;(\boldsymbol{v}, q)] & :=\mu_{\mathrm{eff}}(\nabla \boldsymbol{u}, \nabla \boldsymbol{v})+\sigma(\boldsymbol{u}, \boldsymbol{v})-(p, \nabla \cdot \boldsymbol{v})+(\nabla \cdot \boldsymbol{u}, q), \\
L(\boldsymbol{v}, q) & :=(\boldsymbol{f}, \boldsymbol{v})+(g, q) .
\end{aligned}
$$

In the case $\mu_{\text {eff }}>0$, the weak formulation of problem $11.1,1.2$ reads as: Find $(\boldsymbol{u}, p) \in \boldsymbol{H}_{0}^{1}(\boldsymbol{\Omega}) \times L_{0}^{2}(\boldsymbol{\Omega})$ such that

$$
A[(\boldsymbol{u}, p) ;(\boldsymbol{v}, q)]=L(\boldsymbol{v}, q), \quad \forall(\boldsymbol{v}, q) \in \boldsymbol{H}_{0}^{1}(\boldsymbol{\Omega}) \times L_{0}^{2}(\boldsymbol{\Omega})
$$

Detailed proofs of the well-posedness of problem $\left(2.2\right.$ for $\boldsymbol{f} \in \boldsymbol{L}^{2}(\boldsymbol{\Omega})$ and $g \in L_{0}^{2}(\boldsymbol{\Omega})$ and the corresponding basic theory can be found in, e.g., $2,7,9,20$.

Depending on the given boundary conditions and the regularity of the given data, in the case $\mu_{\mathrm{eff}}=0$ (Darcy limit), the weak solution to the mixed form of problem 1.1 can be sought either in $\boldsymbol{H}_{\mathrm{div}, 0}(\boldsymbol{\Omega}) \times L_{0}^{2}(\boldsymbol{\Omega})$ corresponding to the boundary condition $(1.3)$ or in $\boldsymbol{L}^{2}(\boldsymbol{\Omega}) \times\left[H^{1}(\boldsymbol{\Omega}) \cap L_{0}^{2}(\boldsymbol{\Omega})\right]$.

\subsection{The discrete formulation}

Let us assume that the polygonal computational domain $\boldsymbol{\Omega}$ admits a boundary conforming (fitted) family of triangulations $\left\{\mathcal{T}_{h}\right\}_{h>0}$, i.e., that the discrete domain and the original domain coincide for all $h$. The parameter $h$ denotes a characteristic length of the finite element mesh $\mathcal{T}_{h}$, defined as $h:=\max _{\boldsymbol{T} \in \mathcal{T}_{h}} h_{\boldsymbol{T}}, h_{\boldsymbol{T}}$ being the diameter of the cell $\boldsymbol{T} \in \mathcal{T}_{h}$. Furthermore, we will denote by $\mathcal{G}_{h}$ the set of edges belonging to the boundary $\Gamma$ and with $h_{E}$ the length of $E \in \mathcal{G}_{h}$. Since we assume that $\Omega$ is polygonal, it can be decomposed as the union of $N_{P}$ straight boundary segments and we denote by $\mathcal{C}$ the set of corner nodes of $\mathcal{T}_{h}$.

We will assume that all considered triangulations are nondegenerate, i.e., there exists a constants $C_{\mathrm{SR}}>0$ independent from $h$, such that

$$
\forall h>0, \quad \forall \boldsymbol{T} \in \mathcal{T}_{h}: \quad \frac{h_{\boldsymbol{T}}}{\rho_{\boldsymbol{T}}} \leqslant C_{\mathrm{SR}},
$$

where $\rho_{\boldsymbol{T}}$ is the radius of the largest inscribed sphere in $\boldsymbol{T}$. This property is also known as (shape-) regularity, see 13, p. 124], [17, Def. 1.107]. In particular, it is assumed that there exists a constant $\eta_{0} \geqslant 1$ such that $\frac{h_{E}}{h_{E^{\prime}}} \leqslant \eta_{0}$, for any pair of adjacent edges $E, E^{\prime} \in \mathcal{G}_{h}$. For the validity of the arguments discussed in this paper, we assume that the mesh satisfies also the condition

$$
\eta_{0} \leq 7+4 \sqrt{3} \approx 13.9
$$

Moreover, we require that the triangulation is such that the inner triangles cover an area larger than the boundary ones. Formally, let $B_{h}:=\bigcup_{\boldsymbol{T}: \boldsymbol{T} \cap \Gamma \neq \emptyset} \boldsymbol{T}$ be the union of all triangles which have at least a node on the boundary. We assume that

with $\omega<1$ and independent from $h$.

$$
\left|B_{h}\right| \leqslant \omega|\Omega|
$$

In order to define the discrete problem, let us introduce the quantity $\ell_{\Omega}>0$, representing a typical physical length scale of the problem, and the parameter

$$
\nu:=\mu_{\mathrm{eff}}+\sigma \ell_{\Omega}^{2}
$$

(which has the units of a viscosity). The length $\ell_{\Omega}$ has been introduced mainly for the purpose of consistency of physical units (see, e.g., the discussion in 3]) and it is assumed to satisfy $\ell_{\Omega} \geqslant h_{\boldsymbol{T}}$, for all $\boldsymbol{T} \in\left\{\mathcal{T}_{h}\right\}_{h>0}$. 
Let us now introduce the finite element pair

$$
\begin{aligned}
\boldsymbol{V}_{h}: & =\left\{\boldsymbol{v}_{h} \in \boldsymbol{H}^{1}(\boldsymbol{\Omega}) \cap \boldsymbol{C}^{0}(\overline{\boldsymbol{\Omega}}):\left.\boldsymbol{v}_{h}\right|_{\boldsymbol{T}} \in \mathbb{P}_{1}(\boldsymbol{T}), \quad \forall \boldsymbol{T} \in \mathcal{T}_{h}\right\}, \\
Q_{h} & :=\left\{q_{h} \in L_{0}^{2}(\boldsymbol{\Omega}) \cap C^{0}(\overline{\boldsymbol{\Omega}}):\left.q_{h}\right|_{\boldsymbol{T}} \in \mathbb{P}_{1}(\boldsymbol{T}), \quad \forall \boldsymbol{T} \in \mathcal{T}_{h}\right\},
\end{aligned}
$$

and consider the problem: Find $\left(\boldsymbol{u}_{h}, p_{h}\right) \in \boldsymbol{V}_{h} \times Q_{h}$ such that

$$
A_{h}\left[\left(\boldsymbol{u}_{h}, p_{h}\right) ;\left(\boldsymbol{v}_{h}, q_{h}\right)\right]=L_{h}\left(\boldsymbol{v}_{h}, q_{h}\right), \quad \forall\left(\boldsymbol{v}_{h}, q_{h}\right) \in \boldsymbol{V}_{h} \times Q_{h},
$$

with

$$
\begin{aligned}
A_{h}[(\boldsymbol{u}, p) ;(\boldsymbol{v}, q)]:= & A[(\boldsymbol{u}, p) ;(\boldsymbol{v}, q)]+S_{h, \alpha}^{G L S_{n s}, \mathrm{lhs}}[(\boldsymbol{u}, p),(\boldsymbol{v}, q)]+S_{h, \delta}^{G D, \text { lhs }}[(\boldsymbol{u}, \boldsymbol{v})] \\
& -\left\langle\mu_{\mathrm{eff}} \nabla \boldsymbol{u} \cdot \boldsymbol{n}, \boldsymbol{v}\right\rangle+\langle p \boldsymbol{n}, \boldsymbol{v}\rangle+\left\langle\mu_{\mathrm{eff}} \nabla \boldsymbol{v} \cdot \boldsymbol{n}, \boldsymbol{u}\right\rangle-\langle q \boldsymbol{n}, \boldsymbol{u}\rangle+S_{h, \rho}^{\mathrm{C}, \mathrm{hhs}}(\boldsymbol{u}, \boldsymbol{v}), \\
L_{h}(\boldsymbol{v}, q):= & L(\boldsymbol{v}, q)+S_{h, \alpha}^{G L S_{n s}, \text { rhs }}[(\boldsymbol{v}, q)]+S_{h, \delta}^{G D, \text { rhs }}[\boldsymbol{v}]+S_{h, \rho}^{\mathrm{C}, \mathrm{rhs}}[\boldsymbol{v}] .
\end{aligned}
$$

In (2.8a)-2.8b), $A[(\boldsymbol{u}, p) ;(\boldsymbol{v}, q)]$ is defined in 2.1) and we introduce a stabilization belonging to the nonsymmetric GLS method

$$
\begin{aligned}
& S_{h, \alpha}^{G L S_{n s}, \text { lhs }}[(\boldsymbol{u}, p),(\boldsymbol{v}, q)]:=\alpha \sum_{\boldsymbol{T} \in \mathcal{T}_{h}} \frac{h_{\boldsymbol{T}}^{2}}{\nu}(\sigma \boldsymbol{u}+\nabla p, \sigma \boldsymbol{v}+\nabla q)_{\boldsymbol{T}}, \\
& S_{h, \alpha}^{G L S_{n s}, \text { rhs }}[(\boldsymbol{v}, q)]:=\alpha \sum_{\boldsymbol{T} \in \mathcal{T}_{h}} \frac{h_{\boldsymbol{T}}^{2}}{\nu}(\boldsymbol{f}, \sigma \boldsymbol{v}+\nabla q)_{\boldsymbol{T}},
\end{aligned}
$$

as well as a Grad-Div stabilization

$$
\begin{aligned}
S_{h, \delta}^{G D, \text { lhs }}[(\boldsymbol{u}, \boldsymbol{v})] & :=\delta \nu(\nabla \cdot \boldsymbol{u}, \nabla \cdot \boldsymbol{v}), \\
S_{h, \delta}^{G D, \text { rhs }}[\boldsymbol{v}]: & =\delta \nu(g, \nabla \cdot \boldsymbol{v}) .
\end{aligned}
$$

Additionally, we employ a stabilization term, later referred to as corner stabilization, given by

$$
S_{h, \rho}^{\mathrm{C}, \mathrm{lhs}}[(\boldsymbol{u}, \boldsymbol{v})]:=\rho \nu \sum_{\boldsymbol{x} \in \mathcal{C}} \llbracket \boldsymbol{u} \cdot \boldsymbol{n} \rrbracket(\boldsymbol{x}) \cdot \llbracket \boldsymbol{v} \cdot \boldsymbol{n} \rrbracket(\boldsymbol{x}),
$$

where

$$
\llbracket \boldsymbol{u} \cdot \boldsymbol{n} \rrbracket(\boldsymbol{x}):=\boldsymbol{u}(\boldsymbol{x}) \cdot \boldsymbol{n}_{E}-\boldsymbol{u}(\boldsymbol{x}) \cdot \boldsymbol{n}_{E^{\prime}}=\boldsymbol{u}(\boldsymbol{x}) \cdot\left(\boldsymbol{n}_{E}-\boldsymbol{n}_{E^{\prime}}\right)
$$

denotes the jump of $\boldsymbol{u} \cdot \boldsymbol{n}$ at a corner node $\boldsymbol{x} \in \mathcal{C}:=\left\{\boldsymbol{x}_{c}: \exists E, E^{\prime} \in \Gamma\right.$ such that $\boldsymbol{x}_{c}=E \cap E^{\prime}$ and $\left.\boldsymbol{n}_{E} \neq \boldsymbol{n}_{E^{\prime}}\right\}$, with $E, E^{\prime}$ being the two boundary edges adjacent to $\boldsymbol{x}$.

In the above definitions, $\alpha, \delta$, and $\rho$ are non-negative dimensionless stabilization parameters, which will be assumed to be independent from the mesh size and constant in space. Notice that, in the case of nonhomogeneous boundary conditions, the corresponding right hand sides, consistent with the boundary terms introduced in 2.8a and with the corner stabilization (2.11), have to be included.

The stabilized formulation 2.7) can be regarded as a consistent extension of the pressure stabilizing PetrovGalerkin method (PSPG, [8]) that was introduced in [16] as an unconditionally stable $(\alpha>0)$, non-symmetric formulation of the Stokes problem. This method can be interpreted as a non-symmetric modification of the method proposed in 23, known as Galerkin Least Squares (GLS) method, and for this reason we will refer to it as the 'non-symmetric GLS method'. 
As it will be shown in Section 3 , the stabilization (2.11) is one of the main ingredients of our method. This additional term is used in order to prove robust stability estimates, in particular in the Darcy limit $\left(\mu_{\text {eff }}=0\right.$ or $\sigma \ll 1)$, in absence of the Nitsche penalty term.

Remark 2.1 (On the GLS stabilization terms). Note that for $(2.9)$ since the velocity is approximated using a first order Lagrange finite element space, it holds $-\nabla \cdot\left(\mu_{\mathrm{eff}} \nabla \boldsymbol{u}_{h}\right)=\mathbf{0}$, which allows the simplified expression we are using. The formulation can be analogously extended to the general case of equal finite element pairs $\mathbb{P}_{k} / \mathbb{P}_{k}$ $(k \geqslant 1)$. In these cases, the aforementioned term has to be included in the residuum of the momentum balance equation.

Remark 2.2 (On the Grad-Div stabilization terms). The usage of the Grad-Div stabilization (2.10), originally proposed in 18 (see also, e.g., 24 for further detailed more recent discussions), is motivated here by the need of controlling the $L^{2}$-norm of the divergence of the velocity in the Darcy limit. However this term is also necessary in order to provide stability with respect to the normal velocity on the boundary (see Section 3. Lemma 3.6 for details).

Remark 2.3 (On the discrete setting). The main focus of this paper is the analysis of the penalty-free Nitsche method for the Brinkman model. The non-symmetric GLS, the Grad-Div, as well as the corner stabilization are motivated by our choice of the discrete setting $\left(\mathbb{P}_{1} / \mathbb{P}_{1}\right.$ stabilized finite elements) valid for both the Stokes and the Darcy problems. However, it is worth noticing that the stability estimates which will be proven for the penalty-free Nitsche method and are based on the usage of linear finite elements for velocity and pressure, do not rely on this particular choice of the bulk stabilization, and they can be straightforwardly extended to other approaches (e.g., PSPG or symmetric GLS).

We conclude this section by introducing the norms considered in our analysis:

$$
\begin{aligned}
\|(\boldsymbol{u}, p)\|_{h}^{2}:= & \|\boldsymbol{u}\|^{2}+\sum_{E \in \mathcal{G}_{h}} \theta \frac{\mu_{\mathrm{eff}}}{h_{E}}\|\boldsymbol{u}\|_{0, E}^{2}+\sum_{E \in \mathcal{G}_{h}} \frac{\nu}{h_{E}}\left\|\boldsymbol{u} \cdot \boldsymbol{n}_{E}\right\|_{0, E}^{2}+\rho \nu \sum_{\boldsymbol{x} \in \mathcal{C}}|\llbracket \boldsymbol{u} \cdot \boldsymbol{n} \rrbracket(\boldsymbol{x})|^{2} \\
& +\frac{\|p\|_{0}^{2}}{\nu}+\alpha \sum_{\boldsymbol{T} \in \mathcal{T}_{h}} \frac{h_{\boldsymbol{T}}^{2}}{\nu}\|\nabla p\|_{0, \boldsymbol{T}}^{2}
\end{aligned}
$$

with $\theta:=\frac{\mu_{\text {eff }}}{\nu} \in[0,1]$ and

$$
\|\boldsymbol{u}\|^{2}:=\mu_{\mathrm{eff}}\|\nabla \boldsymbol{u}\|_{0}^{2}+\sigma\|\boldsymbol{u}\|_{0}^{2}+\delta \nu\|\nabla \cdot \boldsymbol{u}\|_{0}^{2} .
$$

As it will be shown in the next section, the scaling by $\theta$ is necessary in order to obtain robust estimates also in the Darcy limit $\left(\mu_{\mathrm{eff}}=0\right)$. We also observe that, if $\sigma=0$ (Stokes limit), the scaling factor is equal to one. In this case, the velocity norm is analogous to the norm used (for the displacement) in the context of the penalty-free Nitsche method for incompressible elasticity [5].

\subsection{Stability and convergence results}

This Section enunciates the main results of this paper, concerning stability and convergence of the proposed penalty-free Nitsche method 2.7). The technical proofs will be discussed in detail in Section 3 .

Theorem (Inf-sup stability) Let $A_{h}\left[\left(\boldsymbol{u}_{h}, p_{h}\right) ;\left(\boldsymbol{v}_{h}, q_{h}\right)\right]$ be the bilinear form defined in $(2.8 \mathrm{a}), \alpha, \delta, \rho>0$, and $\mu_{\mathrm{eff}}, \sigma \geqslant 0$ with $\mu_{\mathrm{eff}}+\sigma>0$. Then there exists a constant $\beta>0$, independent from $h$ and from the physical parameters, such that

$$
\inf _{\left(\boldsymbol{u}_{h}, p_{h}\right) \in \boldsymbol{V}_{h} \times Q_{h} \backslash\{(\mathbf{0}, 0)\}}\left(\sup _{\left(\boldsymbol{v}_{h}, q_{h}\right) \in \boldsymbol{V}_{h} \times Q_{h} \backslash\{(\mathbf{0}, 0)\}}\left(\frac{A_{h}\left[\left(\boldsymbol{u}_{h}, p_{h}\right) ;\left(\boldsymbol{v}_{h}, q_{h}\right)\right]}{\left\|\left(\boldsymbol{u}_{h}, p_{h}\right)\right\|\left\|_{h}\right\|\left(\boldsymbol{v}_{h}, q_{h}\right) \|_{h}}\right)\right) \geqslant \beta .
$$

The inf-sup constant $\beta$ depends only on the stabilization parameters and on the shape regularity of the mesh. 
This statement assesses unconditional stability with respect to the physical parameters, including the limit cases $\sigma=0$ or $\mu_{\mathrm{eff}}=0$. Moreover, we also show that, for small values of the stabilization parameters, it holds $\beta^{-1}=O\left(\alpha^{-1}\left(\rho^{-1}+\delta^{-1}\right)\right)$.

Theorem (A priori error estimate) Let $\alpha, \delta, \rho>0$ and $\mu_{\mathrm{eff}}, \sigma \geqslant 0$ with $\mu_{\mathrm{eff}}+\sigma>0$. Moreover, let (u,p) be the solution of 1.1 with the appropriate boundary conditions and $\left(\boldsymbol{u}_{h}, p_{h}\right)$ be the solution of problem (2.7). Assuming $(\boldsymbol{u}, p) \in \boldsymbol{H}^{2}(\boldsymbol{\Omega}) \times H^{1}(\boldsymbol{\Omega})$, it holds

$$
\left\|\left(\boldsymbol{u}-\boldsymbol{u}_{h}, p-p_{h}\right)\right\|_{h} \leqslant h\left(C_{\boldsymbol{u}}\|\boldsymbol{u}\|_{2}+C_{p}\|p\|_{1}\right)
$$

where $C_{\boldsymbol{u}}$ and $C_{p}$ are independent from $h$ and for small respectively moderate values of stabilization parameters it holds

$$
C_{\boldsymbol{u}}=O\left(\frac{\nu^{\frac{1}{2}}}{\beta}\right), \quad C_{p}=O\left(\frac{1}{\nu^{\frac{1}{2}} \delta^{\frac{1}{2}} \beta}\right) .
$$

With respect to the a priori estimate 2.13 , let us observe that it reduces to standard estimates in the Stokes and in the Darcy limits, for $\sigma=0$ and $\mu_{\text {eff }}=0$, respectively (see, e.g., [3]).

One of the main implications of the above Theorems is therefore the fact that the penalty-free Nitsche formulation possesses a convergence and stability behavior that is comparable to the standard formulation (where essential boundary conditions are imposed in a strong sense) and to the classical (penalty) Nitsche method (see, e.g., 22,26$]$ ).

For the sake of completeness, it is worth observing that, in order to obtain the robust convergence estimate (2.13), the scaling of the stabilization terms with the viscosity $\nu$ defined in $(2.6)$ is a necessary requirement.

Alternative formulations were analyzed, e.g., in [3], for the Brinkman problem with strong imposition of boundary conditions. There, it was shown that stability and optimal error estimates can also be obtained by scaling the stabilization of the Darcy terms with respect to the mesh, replacing $\nu$ by $\nu_{\boldsymbol{T}}:=\mu_{\mathrm{eff}}+\sigma h_{\boldsymbol{T}}^{2}$ on each triangle $\boldsymbol{T} \in \mathcal{T}_{h}$. An analogous scaling as well has been analyzed in 28] (stabilized finite elements for the Darcy equation) and in 26] in the context of a rescaled Brinkman problem with a symmetric Nitsche penalty method (limited to the case $\sigma>0$ ). However, as it will be shown in the next section, the scaling $(2.6)$ is used in order to uniformly control the boundary velocity for $\mu_{\mathrm{eff}}, \sigma \geqslant 0$.

\section{Proof}

In this section, the proofs of the aforementioned Theorems, claiming inf-sup stability and convergence of the proposed method, will be discussed in detail.

\subsection{Preliminaries}

Let us begin by introducing some basic notation and stating a few results that will be utilized in the upcoming analysis.

Let $E$ be an edge of the mesh, and let us denote by $\boldsymbol{T}_{E}$ a triangle attached to $E$. Then, the following discrete trace in ity is valid (see, e.g., 6, (10.3.8)], [14, Lemma 4], [32, pp. 28])

$$
h_{E}^{-1}\|v\|_{L^{2}(E)}^{2} \leqslant c_{\mathrm{DT}}\left(h_{\boldsymbol{T}_{E}}^{-2}\|v\|_{L^{2}\left(\boldsymbol{T}_{E}\right)}^{2}+\|\nabla v\|_{L^{2}\left(\boldsymbol{T}_{E}\right)}^{2}\right), \quad \forall v \in H^{1}\left(\boldsymbol{T}_{E}\right)
$$

where $c_{\mathrm{DT}}>0$ is a constant, only depending on the shape regularity $(2.3)$ of the mesh.

Under the assumption of shape regularity (and assuming $h \leqslant 1$ ), there exists a constant $c_{\mathrm{I}}>0$, independent of $h$ and $\boldsymbol{T}$, such that for all $v_{h} \in \mathbb{P}_{k}(\boldsymbol{T}), k \geqslant 0$, and for all $\boldsymbol{T} \in \mathcal{T}_{h}$ it holds the following inverse inequality 17 , Lemma 1.138]

$$
\left\|\nabla v_{h}\right\|_{L^{2}(\boldsymbol{T})} \leqslant c_{\mathrm{I}} h_{\boldsymbol{T}}^{-1}\left\|v_{h}\right\|_{L^{2}(\boldsymbol{T})} .
$$


Combining (3.1) and $(3.2)$ one can conclude also that there exist a constant $c_{\text {DTI }}$ such that, for any element-wise linear (on the mesh $\mathcal{T}_{h}$ ) function $v_{h}$ it holds (see, e.g., [25, Lemma 3.1], [32, Lemma 2.1])

$$
\begin{aligned}
\left\|v_{h}\right\|_{L^{2}(E)}^{2} & \leqslant c_{\text {DTI }} h_{\boldsymbol{T}_{E}}^{-1}\left\|v_{h}\right\|_{L^{2}\left(\boldsymbol{T}_{E}\right)}^{2}, \\
\sum_{E \in \mathcal{G}_{h}} h_{E}\left\|\nabla v_{h} \cdot \boldsymbol{n}_{E}\right\|_{L^{2}(E)}^{2} & \leqslant c_{\text {DTI }}\left\|\nabla v_{h}\right\|_{L^{2}\left(\boldsymbol{T}_{E}\right)}^{2} .
\end{aligned}
$$

Let us denote with $I_{h}^{S Z}$ the Scott-Zhang interpolator onto the finite element space $\boldsymbol{V}_{h}$ 17 30 , which preserves essential boundary conditions on $\Gamma$. Then, for $l, m \in \mathbb{N}_{0}$ with $1 \leqslant l<\infty$, there exists a constant $c_{\mathrm{SZ}}>0$, depending on the geometry and on the mesh regularity, such that the following approximation properties hold:

$$
\begin{aligned}
& \forall 0 \leqslant m \leqslant 1: \quad\left\|I_{h}^{S Z}(\boldsymbol{v})\right\|_{m, \boldsymbol{\Omega}} \leqslant c_{\mathrm{SZ}}\|\boldsymbol{v}\|_{l, \boldsymbol{\Omega}}, \quad \forall \boldsymbol{v} \in \boldsymbol{H}^{l}(\boldsymbol{\Omega}), \forall h, \\
& \forall l \leqslant 2: \quad \sum_{m=0}^{l} h_{\boldsymbol{T}}^{m}\left\|\boldsymbol{v}-I_{h}^{S Z}(\boldsymbol{v})\right\|_{m, \boldsymbol{T}} \leqslant c_{\mathrm{SZ}} h_{\boldsymbol{T}}^{l}|\boldsymbol{v}|_{l, S(\boldsymbol{T})}, \quad \forall \boldsymbol{v} \in \boldsymbol{H}^{l}(S(\boldsymbol{T})), \forall h, \forall \boldsymbol{T} \in \mathcal{T}_{h},
\end{aligned}
$$

where $S(\boldsymbol{T})$ denotes the union of all cells in $\mathcal{T}_{h}$ which have a vertex in common with $\boldsymbol{T}$.

Finally, let $I_{h}^{L}$ be the Lagrange interpolator onto $\boldsymbol{V}_{h}$. Then, there exists a constant $c_{\mathrm{La}}>0$ such that, there holds (see, e.g., 17, Theorem 1.103]):

$$
\left\|\boldsymbol{v}-I_{h}^{L}(\boldsymbol{v})\right\|_{0, \boldsymbol{T}}+h_{\boldsymbol{T}}\left\|\boldsymbol{v}-I_{h}^{L}(\boldsymbol{v})\right\|_{1, \boldsymbol{T}} \leqslant c_{\mathrm{La}} h_{\boldsymbol{T}}^{2}\|\boldsymbol{v}\|_{2, \boldsymbol{T}}, \quad \forall \boldsymbol{v} \in \boldsymbol{H}^{2}(\boldsymbol{T})
$$

\subsection{Stability}

As next, we will focus on the inf-sup stability of the discrete bilinear form $2.8 \mathrm{a}$ with respect to the meshdependent norm (2.12). Throughout the proofs, the introduced constants depending on the physical parameters or on discretization parameters (mesh size, mesh topology, finite element spaces, stabilization parameters) will be explicated and discussed, in order to allow the reader to follow the derivation in detail and, eventually, to clearly assess the role of the physical parameters within the derived estimates (especially in the limit cases).

The first result concerns the coercivity of the bilinear form 2.8a in a norm which is weaker than 2.12).

Lemma 3.1 (Coercivity in a weaker norm). Let $\alpha>0, \delta, \rho \geqslant 0$ and $\mu_{\mathrm{eff}}, \sigma \geqslant 0$ with $\mu_{\mathrm{eff}}+\sigma>0$. Then there exists a constant $C_{0}=C_{0}(\alpha)>0$, independent from the physical parameters and $h$, such that

$$
A_{h}\left[\left(\boldsymbol{v}_{h}, q_{h}\right) ;\left(\boldsymbol{v}_{h}, q_{h}\right)\right] \geqslant C_{0}\left(\left\|\boldsymbol{v}_{h}\right\|^{2}+\rho \nu \sum_{\boldsymbol{x} \in \mathcal{C}}\left|\llbracket \boldsymbol{v}_{h} \cdot \boldsymbol{n} \rrbracket(\boldsymbol{x})\right|^{2}+\alpha \sum_{\boldsymbol{T} \in \mathcal{T}_{h}} \frac{h_{\boldsymbol{T}}^{2}}{\nu}\left\|\nabla q_{h}\right\|_{0, \boldsymbol{T}}^{2}\right),
$$

for all $\left(\boldsymbol{v}_{h}, q_{h}\right) \in \boldsymbol{V}_{h} \times Q_{h}$.

Proof: Let $\left(\boldsymbol{v}_{h}, q_{h}\right) \in \boldsymbol{V}_{h} \times Q_{h}$, then it is

$$
\begin{aligned}
A_{h}\left[\left(\boldsymbol{v}_{h}, q_{h}\right) ;\left(\boldsymbol{v}_{h}, q_{h}\right)\right]= & \mu_{\mathrm{eff}}\left\|\nabla \boldsymbol{v}_{h}\right\|_{0}^{2}+\sigma\left\|\boldsymbol{v}_{h}\right\|_{0}^{2}+\alpha \sum_{\boldsymbol{T} \in \mathcal{T}_{h}} \frac{h_{\boldsymbol{T}}^{2}}{\nu}\left\|\nabla q_{h}\right\|_{0, \boldsymbol{T}}^{2}+\alpha \sigma^{2} \sum_{\boldsymbol{T} \in \mathcal{T}_{h}} \frac{h_{\boldsymbol{T}}^{2}}{\nu}\left\|\boldsymbol{v}_{h}\right\|_{0, \boldsymbol{T}}^{2} \\
& +2 \alpha \sigma \sum_{\boldsymbol{T} \in \mathcal{T}_{h}} \frac{h_{\boldsymbol{T}}^{2}}{\nu}\left(\nabla q_{h}, \boldsymbol{v}_{h}\right)+\delta \nu\left\|\nabla \cdot \boldsymbol{v}_{h}\right\|_{0}^{2}+\rho \nu \sum_{\boldsymbol{x} \in \mathcal{C}}\left|\llbracket \boldsymbol{v}_{h} \cdot \boldsymbol{n} \rrbracket(\boldsymbol{x})\right|^{2}
\end{aligned}
$$


Using the Cauchy-Schwarz and Young inequalities we obtain

$$
\begin{aligned}
A_{h}\left[\left(\boldsymbol{v}_{h}, q_{h}\right) ;\left(\boldsymbol{v}_{h}, q_{h}\right)\right] \geqslant & \mu_{\mathrm{eff}}\left\|\nabla \boldsymbol{v}_{h}\right\|_{0}^{2}+\sigma\left\|\boldsymbol{v}_{h}\right\|_{0}^{2}+\alpha \sigma\left(1-\frac{1}{\varepsilon}\right) \sum_{\boldsymbol{T} \in \mathcal{T}_{h}} \frac{\sigma h_{\boldsymbol{T}}^{2}}{\nu}\left\|\boldsymbol{v}_{h}\right\|_{0, \boldsymbol{T}}^{2} \\
& +\delta \nu\left\|\nabla \cdot \boldsymbol{v}_{h}\right\|_{0}^{2}+\rho \nu \sum_{\boldsymbol{x} \in \mathcal{C}}\left|\llbracket \boldsymbol{v}_{h} \cdot \boldsymbol{n} \rrbracket(\boldsymbol{x})\right|^{2}+(1-\varepsilon) \alpha \sum_{\boldsymbol{T} \in \mathcal{T}_{h}} \frac{h_{\boldsymbol{T}}^{2}}{\nu}\left\|\nabla q_{h}\right\|_{0, \boldsymbol{T}}^{2},
\end{aligned}
$$

and since $\frac{\sigma h_{T}^{2}}{\nu} \leqslant \frac{\sigma \ell_{\Omega}^{2}}{\nu} \leqslant 1$ we get with $\epsilon<1$ the bound

$$
\begin{aligned}
A_{h}\left[\left(\boldsymbol{v}_{h}, q_{h}\right) ;\left(\boldsymbol{v}_{h}, q_{h}\right)\right] \geqslant & \mu_{\mathrm{eff}}\left\|\nabla \boldsymbol{v}_{h}\right\|_{0}^{2}+\left(1+\alpha-\frac{\alpha}{\varepsilon}\right) \sigma\left\|\boldsymbol{v}_{h}\right\|_{0}^{2}+\delta \nu\left\|\nabla \cdot \boldsymbol{v}_{h}\right\|_{0}^{2} \\
& +\rho \nu \sum_{\boldsymbol{x} \in \mathcal{C}}\left|\llbracket \boldsymbol{v}_{h} \cdot \boldsymbol{n} \rrbracket(\boldsymbol{x})\right|^{2}+(1-\varepsilon) \alpha \sum_{\boldsymbol{T} \in \mathcal{T}_{h}} \frac{h_{\boldsymbol{T}}^{2}}{\nu}\left\|\nabla q_{h}\right\|_{0, \boldsymbol{T}}^{2} .
\end{aligned}
$$

In order to obtain the stability estimate, we choose $\varepsilon$ such that $\frac{\alpha}{\alpha+1}<\varepsilon<1$, so that $\left(1+\alpha-\frac{\alpha}{\varepsilon}\right)$ and $(1-\varepsilon)$ are strictly positive. Taking $\varepsilon:=\left(\sqrt{\frac{\alpha^{2}}{4}+\alpha}-\frac{\alpha}{2}\right)$ these two coefficients coincide, and we obtain

$$
\begin{aligned}
A_{h}\left[\left(\boldsymbol{v}_{h}, q_{h}\right) ;\left(\boldsymbol{v}_{h}, q_{h}\right)\right] \geqslant & \mu_{\mathrm{eff}}\left\|\nabla \boldsymbol{v}_{h}\right\|_{0}^{2}+\delta \nu\left\|\nabla \cdot \boldsymbol{v}_{h}\right\|_{0}^{2}+\rho \nu \sum_{\boldsymbol{x} \in \mathcal{C}}\left|\llbracket \boldsymbol{v}_{h} \cdot \boldsymbol{n} \rrbracket(\boldsymbol{x})\right|^{2} \\
& +(1-\varepsilon)\left(\sigma\left\|\boldsymbol{v}_{h}\right\|_{0}^{2}+\alpha \sum_{\boldsymbol{T} \in \mathcal{T}_{h}} \frac{h_{\boldsymbol{T}}^{2}}{\nu}\left\|\nabla q_{h}\right\|_{0, \boldsymbol{T}}^{2}\right) .
\end{aligned}
$$

The proof is concluded defining

$$
C_{0}:=1-\varepsilon=1-\sqrt{\frac{\alpha^{2}}{4}+\alpha}+\frac{\alpha}{2}
$$

Remark 3.1 (On the behavior of $C_{0}$ ). Notice that the constant $C_{0}$ introduced in 3.7 is a decreasing function of $\alpha$ satisfying $C_{0}(0)=1$. In particular, $C_{0}=O(1)$ for small and moderate values of $\alpha$. Note that the estimate (3.6) holds also (trivially) for $\alpha=0$.

The following Lemma provides stability in the $L^{2}$-norm of the pressure.

Lemma 3.2 (Pressure control). Let $\alpha>0, \delta, \rho \geqslant 0$, and $\mu_{\mathrm{eff}}, \sigma \geqslant 0$ with $\mu_{\mathrm{eff}}+\sigma>0$. Then, there exists a constant $C_{1}=C_{1}(\alpha, \delta)>0$, independent from the physical parameters and $h$, such that, for all $\left(\boldsymbol{u}_{h}, p_{h}\right) \in$ $\boldsymbol{V}_{h} \times Q_{h}$, we can find a function $\boldsymbol{v}_{h} \in \boldsymbol{V}_{h}$ that satisfies

$$
A_{h}\left[\left(\boldsymbol{u}_{h}, p_{h}\right) ;\left(\boldsymbol{v}_{h}, 0\right)\right] \geqslant \frac{1}{2} \frac{\left\|p_{h}\right\|_{0}^{2}}{\nu}-C_{1}\left(\left\|\boldsymbol{u}_{h}\right\|^{2}+\alpha \sum_{\boldsymbol{T} \in \mathcal{T}_{h}} \frac{h_{\boldsymbol{T}}^{2}}{\nu}\left\|\nabla p_{h}\right\|_{0, \boldsymbol{T}}^{2}+\sum_{E \in \mathcal{G}_{h}} \theta \frac{\mu_{\mathrm{eff}}}{h_{E}}\left\|\boldsymbol{u}_{h}\right\|_{0, E}^{2}\right) .
$$

Proof: Let $\left(\boldsymbol{u}_{h}, p_{h}\right) \in \boldsymbol{V}_{h} \times Q_{h}$. Since $p_{h} \in Q_{h} \subset L_{0}^{2}(\boldsymbol{\Omega})$ (due to conformity), there exists exactly one $\boldsymbol{v}_{p_{h}} \in \boldsymbol{H}_{0}^{1}(\boldsymbol{\Omega})$ and a dimensionless constant $\hat{c}_{\boldsymbol{\Omega}}$ (that only depends on $\boldsymbol{\Omega}$ ) such that 20, Corollary 2.4]

$$
\begin{gathered}
\nabla \cdot \boldsymbol{v}_{p_{h}}=-\frac{1}{\nu} p_{h}, \\
\left\|\nabla \boldsymbol{v}_{p_{h}}\right\|_{0} \leqslant \frac{\hat{c_{\boldsymbol{\Omega}}}}{\nu}\left\|p_{h}\right\|_{0} .
\end{gathered}
$$


Let now $\boldsymbol{v}_{h}:=I_{h}^{S Z}\left(\boldsymbol{v}_{p_{h}}\right)$ be the Scott-Zhang interpolator of the function $\boldsymbol{v}_{p_{h}}$ onto $\boldsymbol{V}_{h}$. Due to the $H^{1}$-stability of the Scott-Zhang interpolator (3.4a), property (3.9b), and the Poincaré inequality 17, Lemma B.61], there also holds

$$
\left\|\nabla \boldsymbol{v}_{h}\right\|_{0} \leqslant \frac{c_{\boldsymbol{\Omega}}}{\nu}\left\|p_{h}\right\|_{0} \quad \text { and } \quad\left\|\boldsymbol{v}_{h}\right\|_{0} \leqslant \frac{\ell_{\Omega} c_{\boldsymbol{\Omega}}}{\nu}\left\|p_{h}\right\|_{0}
$$

with a constant $c_{\boldsymbol{\Omega}}$ that only depends on the domain and on the regularity of the mesh. Moreover, according to $3.4 \mathrm{~b}$, it holds

$$
\sum_{\boldsymbol{T} \in \mathcal{T}_{h}} \frac{1}{h_{\boldsymbol{T}}^{2}}\left\|\boldsymbol{v}_{p_{h}}-\boldsymbol{v}_{h}\right\|_{0, \boldsymbol{T}}^{2} \leqslant \sum_{\boldsymbol{T} \in \mathcal{T}_{h}} \frac{1}{h_{\boldsymbol{T}}^{2}} c_{\mathrm{SZ}}^{2} h_{\boldsymbol{T}}^{2}\left\|\nabla \boldsymbol{v}_{p_{h}}\right\|_{0, S(\boldsymbol{T})}^{2} \leqslant{\widetilde{c_{\mathrm{SZ}}}}^{2}\left\|\nabla \boldsymbol{v}_{p_{h}}\right\|_{0}^{2}
$$

with ${\widetilde{c_{\mathrm{SZ}}}}^{2}:=c_{\mathrm{SZ}}^{2}\left(\max _{\boldsymbol{T} \in \mathcal{T}_{h}} \# S(\boldsymbol{T})\right)$. Here, \#S(T) denotes the number of triangles contained in $S(\boldsymbol{T})$ which depends on the regularity of the mesh.

Since the Scott-Zhang interpolator preserves essential boundary conditions, it holds $\boldsymbol{v}_{h} \in \boldsymbol{H}_{0}^{1}(\boldsymbol{\Omega}) \cap \boldsymbol{V}_{h}$ such that the boundary terms involving $\boldsymbol{v}_{h}$ vanish. Using the decomposition $\boldsymbol{v}_{h}=\boldsymbol{v}_{p_{h}}-\left(\boldsymbol{v}_{p_{h}}-\boldsymbol{v}_{h}\right)$ and integration by parts for the term $\left(\boldsymbol{v}_{p_{h}}-\boldsymbol{v}_{h}\right) \in \boldsymbol{H}_{0}^{1}(\boldsymbol{\Omega})$ we get

$$
\begin{aligned}
A_{h}\left[\left(\boldsymbol{u}_{h}, p_{h}\right) ;\left(\boldsymbol{v}_{h}, 0\right)\right]= & \mu_{\mathrm{eff}}\left(\nabla \boldsymbol{u}_{h}, \nabla \boldsymbol{v}_{h}\right)+\sigma\left(\boldsymbol{u}_{h}, \boldsymbol{v}_{h}\right)-\left(p_{h}, \nabla \cdot \boldsymbol{v}_{h}\right) \\
& +\alpha \sum_{\boldsymbol{T} \in \mathcal{T}_{h}} \frac{h_{\boldsymbol{T}}^{2}}{\nu}\left(\sigma \boldsymbol{u}_{h}+\nabla p_{h}, \sigma \boldsymbol{v}_{h}\right)_{\boldsymbol{T}}+\delta \nu\left(\nabla \cdot \boldsymbol{u}_{h}, \nabla \cdot \boldsymbol{v}_{h}\right) \\
& +\left\langle\mu_{\mathrm{eff}} \nabla \boldsymbol{v}_{h} \cdot \boldsymbol{n}, \boldsymbol{u}_{h}\right\rangle \\
= & \mu_{\mathrm{eff}}\left(\nabla \boldsymbol{u}_{h}, \nabla \boldsymbol{v}_{h}\right)+\sigma\left(\boldsymbol{u}_{h}, \boldsymbol{v}_{h}\right)-\left(p_{h}, \nabla \cdot \boldsymbol{v}_{p_{h}}\right)-\left(\nabla p_{h}, \boldsymbol{v}_{p_{h}}-\boldsymbol{v}_{h}\right) \\
& +\alpha \sum_{\boldsymbol{T} \in \mathcal{T}_{h}} \frac{h_{\boldsymbol{T}}^{2}}{\nu}\left(\sigma \boldsymbol{u}_{h}+\nabla p_{h}, \sigma \boldsymbol{v}_{h}\right)_{\boldsymbol{T}}+\delta \nu\left(\nabla \cdot \boldsymbol{u}_{h}, \nabla \cdot \boldsymbol{v}_{h}\right) \\
& +\left\langle\mu_{\mathrm{eff}} \nabla \boldsymbol{v}_{h} \cdot \boldsymbol{n}, \boldsymbol{u}_{h}\right\rangle .
\end{aligned}
$$

Using the Cauchy-Schwarz inequality, the equality (3.9a), the inequality (3.11) and $\left\|\nabla \cdot \boldsymbol{v}_{h}\right\|_{0} \leqslant n^{\frac{1}{2}}\left\|\nabla \boldsymbol{v}_{h}\right\|_{0}$, we obtain

$$
\begin{aligned}
A_{h}\left[\left(\boldsymbol{u}_{h}, p_{h}\right) ;\left(\boldsymbol{v}_{h}, 0\right)\right] \geqslant & -\mu_{\mathrm{eff}}^{\frac{1}{2}}\left(\mu_{\mathrm{eff}}^{\frac{1}{2}}\left\|\nabla \boldsymbol{u}_{h}\right\|_{0}\right)\left\|\nabla \boldsymbol{v}_{h}\right\|_{0}-\sigma^{\frac{1}{2}}\left(\sigma^{\frac{1}{2}}\left\|\boldsymbol{u}_{h}\right\|_{0}\right)\left\|\boldsymbol{v}_{h}\right\|_{0}+\frac{\left\|p_{h}\right\|_{0}^{2}}{\nu} \\
& -c_{\mathrm{SZ}}\left(\sum_{\boldsymbol{T} \in \mathcal{T}_{h}} h_{\boldsymbol{T}}^{2}\left\|\nabla p_{h}\right\|_{0, \boldsymbol{T}}^{2}\right)^{\frac{1}{2}}\left\|\nabla \boldsymbol{v}_{p_{h}}\right\|_{0}-\underbrace{\alpha \sigma \sum_{\boldsymbol{T} \in \mathcal{T}_{h}} \frac{\sigma h_{\boldsymbol{T}}^{2}}{\nu}\left\|\boldsymbol{u}_{h}\right\|_{0, \boldsymbol{T}}\left\|\boldsymbol{v}_{h}\right\|_{0, \boldsymbol{T}}}_{=: \mathcal{T}_{1}} \\
& -\underbrace{\alpha \sum_{\boldsymbol{T} \in \mathcal{T}_{h}} \frac{\sigma h_{\boldsymbol{T}}^{2}}{\nu}\left\|\nabla p_{h}\right\|_{0, \boldsymbol{T}}\left\|\boldsymbol{v}_{h}\right\|_{0, \boldsymbol{T}}}_{=: \mathcal{T}_{2}}-\delta \nu^{\frac{1}{2}}\left\|\nabla \cdot \boldsymbol{u}_{h}\right\|_{0} n^{\frac{1}{2}} \nu^{\frac{1}{2}}\left\|\nabla \boldsymbol{v}_{h}\right\|_{0} \\
& +\left\langle\mu_{\mathrm{eff}} \nabla \boldsymbol{v}_{h} \cdot \boldsymbol{n}, \boldsymbol{u}_{h}\right\rangle .
\end{aligned}
$$


The terms $\mathcal{T}_{1}$ and $\mathcal{T}_{2}$ introduced above can be estimated using the Cauchy-Schwarz inequality and the inequalities (3.10), yielding

$$
\mathcal{T}_{1}=\alpha \sigma \sum_{\boldsymbol{T} \in \mathcal{T}_{h}} \underbrace{\frac{\sigma h_{\boldsymbol{T}}^{2}}{\nu}}_{\leqslant 1}\left\|\boldsymbol{u}_{h}\right\|_{0, \boldsymbol{T}}\left\|\boldsymbol{v}_{h}\right\|_{0, \boldsymbol{T}} \leqslant \alpha \sigma^{\frac{1}{2}}\left\|\boldsymbol{u}_{h}\right\|_{0} \sigma^{\frac{1}{2}}\left\|\boldsymbol{v}_{h}\right\|_{0} \leqslant c_{\boldsymbol{\Omega}} \alpha \sigma^{\frac{1}{2}}\left\|\boldsymbol{u}_{h}\right\|_{0} \frac{\left\|p_{h}\right\|_{0}}{\nu^{\frac{1}{2}}}
$$

and

$$
\begin{aligned}
\mathcal{T}_{2} & =\alpha \sum_{\boldsymbol{T} \in \mathcal{T}_{h}} \sigma^{\frac{1}{2}}\left(\frac{h_{\boldsymbol{T}}^{2}}{\nu}\right)^{\frac{1}{2}} \underbrace{\left(\frac{\sigma h_{\boldsymbol{T}}^{2}}{\nu}\right)^{\frac{1}{2}}}_{\leqslant 1}\left\|\nabla p_{h}\right\|_{0, \boldsymbol{T}}\left\|\boldsymbol{v}_{h}\right\|_{0, \boldsymbol{T}} \leqslant \alpha\left(\sum_{\boldsymbol{T} \in \mathcal{T}_{h}} \frac{h_{\boldsymbol{T}}^{2}}{\nu}\left\|\nabla p_{h}\right\|_{0, \boldsymbol{T}}^{2}\right)^{\frac{1}{2}} \sigma^{\frac{1}{2}}\left\|\boldsymbol{v}_{h}\right\|_{0} \\
& \leqslant \alpha\left(\sum_{\boldsymbol{T} \in \mathcal{T}_{h}} \frac{h_{\boldsymbol{T}}^{2}}{\nu}\left\|\nabla p_{h}\right\|_{0, \boldsymbol{T}}^{2}\right)^{\frac{1}{2}} \sigma^{\frac{1}{2}} \frac{\ell_{\Omega} c_{\boldsymbol{\Omega}}}{\nu}\left\|p_{h}\right\|_{0} \leqslant c_{\boldsymbol{\Omega}} \alpha\left(\sum_{\boldsymbol{T} \in \mathcal{T}_{h}} \frac{h_{\boldsymbol{T}}^{2}}{\nu}\left\|\nabla p_{h}\right\|_{0, \boldsymbol{T}}^{2}\right)^{\frac{1}{2}} \frac{\left\|p_{h}\right\|_{0}}{\nu^{\frac{1}{2}}} .
\end{aligned}
$$

For the boundary term we apply the Cauchy-Schwarz inequality, the inequality $3.3 \mathrm{~b}$, and the estimate 3.10 to derive

$$
\begin{aligned}
\left\langle\mu_{\mathrm{eff}} \nabla \boldsymbol{v}_{h} \cdot \boldsymbol{n}, \boldsymbol{u}_{h}\right\rangle & \leqslant\left(\sum_{E \in \mathcal{G}_{h}} \mu_{\mathrm{eff}} h_{E}\left\|\nabla \boldsymbol{v}_{h} \cdot \boldsymbol{n}_{E}\right\|_{0, E}^{2}\right)^{\frac{1}{2}}\left(\sum_{E \in \mathcal{G}_{h}} \frac{\mu_{\mathrm{eff}}}{h_{E}}\left\|\boldsymbol{u}_{h}\right\|_{0, E}^{2}\right)^{\frac{1}{2}} \\
& \leqslant c_{\mathrm{DTI}}^{\frac{1}{2}}\left(\mu_{\mathrm{eff}} \sum_{E \in \mathcal{G}_{h}}\left\|\nabla \boldsymbol{v}_{h}\right\|_{0, \boldsymbol{T}_{E}}^{2}\right)^{\frac{1}{2}}\left(\sum_{E \in \mathcal{G}_{h}} \frac{\mu_{\mathrm{eff}}}{h_{E}}\left\|\boldsymbol{u}_{h}\right\|_{0, E}^{2}\right)^{\frac{1}{2}} \\
& \leqslant c_{\boldsymbol{\Omega}} c_{\mathrm{DTI}}^{\frac{1}{2}} \frac{\left\|p_{h}\right\|_{0}}{\nu^{\frac{1}{2}}} \underbrace{\left(\frac{\mu_{\mathrm{eff}}}{\nu}\right)^{\frac{1}{2}}}_{=\theta^{\frac{1}{2}}}\left(\sum_{E \in \mathcal{G}_{h}} \frac{\mu_{\mathrm{eff}}}{h_{E}}\left\|\boldsymbol{u}_{h}\right\|_{0, E}^{2}\right)^{\frac{1}{2}} .
\end{aligned}
$$

Inserting 3.13), 3.14, and 3.15) into 3.12, using the estimates 3.10 and 3.9b), and rearranging the terms one obtains

$$
\begin{aligned}
A_{h}\left[\left(\boldsymbol{u}_{h}, p_{h}\right) ;\left(\boldsymbol{v}_{h}, 0\right)\right] \geqslant & \frac{\left\|p_{h}\right\|_{0}^{2}}{\nu}-c_{\boldsymbol{\Omega}}\left(\mu_{\mathrm{eff}}^{\frac{1}{2}}\left\|\nabla \boldsymbol{u}_{h}\right\|_{0}\right) \frac{\left\|p_{h}\right\|_{0}}{\nu^{\frac{1}{2}}}-c_{\boldsymbol{\Omega}}(1+\alpha) \sigma^{\frac{1}{2}}\left\|\boldsymbol{u}_{h}\right\|_{0} \frac{\left\|p_{h}\right\|_{0}}{\nu^{\frac{1}{2}}} \\
& -\left(\frac{c_{\mathrm{SZ}} \hat{c}_{\boldsymbol{\Omega}}}{\sqrt{\alpha}}+c_{\boldsymbol{\Omega}} \sqrt{\alpha}\right)\left(\alpha \sum_{\boldsymbol{T} \in \mathcal{T}_{h}} \frac{h_{\boldsymbol{T}}^{2}}{\nu}\left\|\nabla p_{h}\right\|_{0, \boldsymbol{T}}^{2}\right)^{\frac{1}{2}} \frac{\left\|p_{h}\right\|_{0}}{\nu^{\frac{1}{2}}} \\
& -c_{\boldsymbol{\Omega}}(n \delta)^{\frac{1}{2}}\left(\delta \nu\left\|\nabla \cdot \boldsymbol{u}_{h}\right\|_{0}^{2}\right)^{\frac{1}{2}} \frac{\left\|p_{h}\right\|_{0}}{\nu^{\frac{1}{2}}}-c_{\boldsymbol{\Omega}} c_{\mathrm{DTI}}^{\frac{1}{2}}\left(\sum_{E \in \mathcal{G}_{h}} \theta \frac{\mu_{\mathrm{eff}}}{h_{E}}\left\|\boldsymbol{u}_{h}\right\|_{0, E}^{2}\right)^{\frac{1}{2}} \frac{\left\|p_{h}\right\|_{0}}{\nu^{\frac{1}{2}}}
\end{aligned}
$$

We now define

$$
C_{1}^{\prime}:=\max \left\{c_{\boldsymbol{\Omega}}^{2}(1+\alpha)^{2}, \frac{\left(c_{\mathrm{SZ}} \hat{c}_{\boldsymbol{\Omega}}+c_{\boldsymbol{\Omega}} \alpha\right)^{2}}{\alpha}, c_{\boldsymbol{\Omega}}^{2} n \delta, c_{\boldsymbol{\Omega}}^{2} c_{\mathrm{DTI}}\right\}
$$


and using the Young inequality we obtain the estimate

$$
A_{h}\left[\left(\boldsymbol{u}_{h}, p_{h}\right) ;\left(\boldsymbol{v}_{h}, 0\right)\right] \geqslant \frac{1}{2} \frac{\left\|p_{h}\right\|_{0}^{2}}{\nu}-C_{1}\left(\left\|\boldsymbol{u}_{h}\right\|^{2}+\alpha \sum_{\boldsymbol{T} \in \mathcal{T}_{h}} \frac{h_{\boldsymbol{T}}^{2}}{\nu}\left\|\nabla p_{h}\right\|_{0, \boldsymbol{T}}^{2}+\sum_{E \in \mathcal{G}_{h}} \theta \frac{\mu_{\mathrm{eff}}}{h_{E}}\left\|\boldsymbol{u}_{h}\right\|_{0, E}^{2}\right),
$$

with $C_{1}:=3 C_{1}^{\prime}$.

Remark 3.2 (On the behavior of $C_{1}$ ). The constant $C_{1}$ in (3.8) depends only on the stabilization parameters $\alpha$ and $\delta$, on the domain $\boldsymbol{\Omega}$, and on the discretization (through the constants $n, c_{\boldsymbol{\Omega}}, \hat{c}_{\boldsymbol{\Omega}}, c_{\mathrm{SZ}}$, and $c_{\mathrm{DTI}}$ ). In particular $C_{1} \sim \frac{1}{\alpha}$ for $\alpha \ll 1$.

The next step concerns the stability of the proposed formulation with respect to the boundary velocity. To this aim, we will show that the skew-symmetric Nitsche terms in 2.8a yield a stable formulation by defining two particular test functions that provide control of the boundary norms of the velocity.

The construction of the first test function and its main properties are stated in the following Lemma.

Lemma 3.3. For any $\boldsymbol{u}_{h} \in \boldsymbol{V}_{h}$ we define $\boldsymbol{w}_{h}^{\boldsymbol{u}_{h}} \in \boldsymbol{V}_{h}$ such that, for each mesh node $\boldsymbol{x}$, it holds

$$
\boldsymbol{w}_{h}^{\boldsymbol{u}_{h}}(\boldsymbol{x}):= \begin{cases}\boldsymbol{u}_{h}(\boldsymbol{x}), & \text { for } \boldsymbol{x} \in \Gamma, \\ \mathbf{0}, & \text { for } \boldsymbol{x} \in \overline{\boldsymbol{\Omega}} \backslash \Gamma .\end{cases}
$$

Then the function $\boldsymbol{w}_{h}^{\boldsymbol{u}_{h}}$ satisfies the following properties:

(1) There exist two positive constants $c_{0}$ and $c_{1}$, depending only on the regularity of the mesh, such that

$$
\left\langle\mu_{\mathrm{eff}} \nabla \boldsymbol{w}_{h}^{\boldsymbol{u}_{h}} \cdot \boldsymbol{n}, \boldsymbol{u}_{h}\right\rangle \geqslant c_{0} \sum_{E \in \mathcal{G}_{h}} \frac{\mu_{\mathrm{eff}}}{h_{E}}\left\|\boldsymbol{u}_{h}\right\|_{0, E}^{2}-c_{1} \mu_{\mathrm{eff}}\left\|\nabla \boldsymbol{u}_{h}\right\|_{0}^{2} .
$$

(2) There exists a constant $c_{2}>0$, depending only on the regularity of the mesh, such that

$$
\mu_{\mathrm{eff}}\left\|\nabla \boldsymbol{w}_{h}^{\boldsymbol{u}_{h}}\right\|_{0}^{2} \leqslant c_{2} \sum_{E \in \mathcal{G}_{h}} \frac{\mu_{\mathrm{eff}}}{h_{E}}\left\|\boldsymbol{u}_{h}\right\|_{0, E}^{2} .
$$

(3) There exists a constant $c_{3}>0$, depending on the mesh regularity, such that

$$
\left\|\boldsymbol{w}_{h}^{\boldsymbol{u}_{h}}\right\|_{0} \leqslant c_{3}\left\|\boldsymbol{u}_{h}\right\|_{0}
$$

(4) There exists a constant $\widetilde{c_{3}}>0$, depending on the mesh regularity, such that

$$
\left\|\boldsymbol{w}_{h}^{\boldsymbol{u}_{h}}\right\|_{0, \boldsymbol{T}}^{2} \leqslant \widetilde{c_{3}} h_{\boldsymbol{T}}^{2}\left\|\nabla \boldsymbol{w}_{h}^{\boldsymbol{u}_{h}}\right\|_{0, \boldsymbol{T}}^{2}, \quad \forall \boldsymbol{T} \in \mathcal{T}_{h} .
$$

Proof: Let us consider $\boldsymbol{u}_{h} \in \boldsymbol{V}_{h}$ and let $\boldsymbol{w}_{h}^{\boldsymbol{u}_{h}} \in \boldsymbol{V}_{h}$ be defined as in (3.16). In the following proof, for an edge $E \in \mathcal{G}_{h}$ with vertices $\boldsymbol{x}_{1}$ and $\boldsymbol{x}_{2}$ we will denote the (unique) attached triangle by $\boldsymbol{T}_{E}=\operatorname{conv}\left\{\boldsymbol{x}_{0}, \boldsymbol{x}_{1}, \boldsymbol{x}_{2}\right\}$.

(1) In order to prove (3.17), let us introduce $\boldsymbol{w}_{E}: \mathbb{R}^{2} \rightarrow \mathbb{R}^{2}$ as the linear function that coincides with $\boldsymbol{w}_{h}^{\boldsymbol{u}_{h}}$ in $\boldsymbol{T}_{E}$ and extends it everywhere in $\mathbb{R}^{2}$. Since $\boldsymbol{w}_{h}^{\boldsymbol{u}_{h}}\left(\boldsymbol{x}_{0}\right)=\mathbf{0}$, it holds

$$
\left.\left(\nabla \boldsymbol{w}_{h}^{\boldsymbol{u}_{h}} \cdot \boldsymbol{n}_{E}\right)\right|_{E}=\frac{\boldsymbol{w}_{E}\left(\boldsymbol{x}_{\perp}\right)}{h_{E, \perp}},
$$

where $\boldsymbol{x}_{\perp}$ is the perpendicular foot of the vertex $\boldsymbol{x}_{0}$ and $h_{E, \perp}$ is the height of the triangle $\boldsymbol{T}_{E}$ with respect to the edge $E$. Depending on the shape of $\boldsymbol{T}_{E}, \boldsymbol{x}_{\perp}$ might fall inside or outside the edge $E$. Formally, there exists an $a \in \mathbb{R}$, such that

$$
\boldsymbol{x}_{\perp}=a \boldsymbol{x}_{1}+(1-a) \boldsymbol{x}_{2}, \quad|a|+|1-a| \leqslant M
$$


where $M>0$ depends only on the mesh regularity constant. Hence, by adding and subtracting $\boldsymbol{u}_{h}$ we can reformulate

$$
\begin{aligned}
\left\langle\nabla \boldsymbol{w}_{h}^{\boldsymbol{u}_{h}} \cdot \boldsymbol{n}, \boldsymbol{u}_{h}\right\rangle_{E} & =\frac{1}{h_{E, \perp}}\left\langle\boldsymbol{w}_{E}\left(\boldsymbol{x}_{\perp}\right), \boldsymbol{u}_{h}\right\rangle_{E}=\frac{1}{h_{E, \perp}}\left(\left\langle\boldsymbol{u}_{h}, \boldsymbol{u}_{h}\right\rangle_{E}-\left\langle\boldsymbol{u}_{h}-\boldsymbol{w}_{E}\left(\boldsymbol{x}_{\perp}\right), \boldsymbol{u}_{h}\right\rangle_{E}\right) \\
& =\frac{h_{E}}{h_{E, \perp}}\left(\frac{1}{h_{E}}\left\|\boldsymbol{u}_{h}\right\|_{0, E}^{2}\right)-\frac{1}{h_{E, \perp}}\left\langle\boldsymbol{u}_{h}-\boldsymbol{w}_{E}\left(\boldsymbol{x}_{\perp}\right), \boldsymbol{u}_{h}\right\rangle_{E} .
\end{aligned}
$$

Exploiting (3.21), the linearity of $\boldsymbol{w}_{E}$, and the fact that $\boldsymbol{w}_{E}$ coincindes with $\boldsymbol{u}_{h}$ on $E$, we get, for all $\boldsymbol{x} \in E$,

$$
\begin{aligned}
\left|\boldsymbol{u}_{h}(\boldsymbol{x})-\boldsymbol{w}_{E}\left(\boldsymbol{x}_{\perp}\right)\right| & =\left|\boldsymbol{u}_{h}(\boldsymbol{x})-\left(a \boldsymbol{u}_{h}\left(\boldsymbol{x}_{1}\right)+(1-a) \boldsymbol{u}_{h}\left(\boldsymbol{x}_{2}\right)\right)\right| \\
& \leqslant\left(|a|\left|\boldsymbol{u}_{h}(\boldsymbol{x})-\boldsymbol{u}_{h}\left(\boldsymbol{x}_{1}\right)\right|+|1-a|\left|\boldsymbol{u}_{h}(\boldsymbol{x})-\boldsymbol{u}_{h}\left(\boldsymbol{x}_{2}\right)\right|\right) \\
& \leqslant M\left(\left|\boldsymbol{u}_{h}(\boldsymbol{x})-\boldsymbol{u}_{h}\left(\boldsymbol{x}_{1}\right)\right|+\left|\boldsymbol{u}_{h}(\boldsymbol{x})-\boldsymbol{u}_{h}\left(\boldsymbol{x}_{2}\right)\right|\right) \\
& \leqslant 2 M h_{E}\left|\left(\nabla \boldsymbol{u}_{h}\right)\right|_{\boldsymbol{T}_{E}} \mid
\end{aligned}
$$

where $|\cdot|$ stands for the Euclidean norm. Since $\nabla \boldsymbol{u}_{h}$ is constant on $\boldsymbol{T}_{E}$, it holds also

$$
\left\|\nabla \boldsymbol{u}_{h}\right\|_{0, \boldsymbol{T}_{E}}=\left|\boldsymbol{T}_{E}\right|^{\frac{1}{2}}\left|\left(\nabla \boldsymbol{u}_{h}\right)\right|_{\boldsymbol{T}_{E}} \mid,
$$

from which we deduce

$$
\left|\left(\nabla \boldsymbol{u}_{h}\right)\right|_{\boldsymbol{T}_{E}} \mid \leqslant c h_{E}^{-1}\left\|\nabla \boldsymbol{u}_{h}\right\|_{0, \boldsymbol{T}_{E}}
$$

where the constant $c>0$ only depends the regularity of the mesh. The above arguments allow to conclude

$$
\left\|\boldsymbol{u}_{h}-\boldsymbol{w}_{E}\left(\boldsymbol{x}_{\perp}\right)\right\|_{0, E} \leqslant h_{E}^{\frac{1}{2}} \max _{\boldsymbol{x} \in E}\left|\boldsymbol{u}_{h}(\boldsymbol{x})-\boldsymbol{w}_{E}\left(\boldsymbol{x}_{\perp}\right)\right| \leqslant 2 M h_{E}^{\frac{3}{2}}\left|\left(\nabla \boldsymbol{u}_{h}\right)\right|_{\boldsymbol{T}_{E}} \mid \leqslant c_{\Gamma} h_{E}^{\frac{1}{2}}\left\|\nabla \boldsymbol{u}_{h}\right\|_{0, \boldsymbol{T}_{E}},
$$

with $c_{\Gamma}:=2 M c$. Thus, applying the Cauchy-Schwarz inequality, the inequality (3.24), and the Young inequality yields

$$
\left\langle\boldsymbol{u}_{h}-\boldsymbol{w}_{E}\left(\boldsymbol{x}_{\perp}\right), \boldsymbol{u}_{h}\right\rangle_{E} \leqslant c_{\Gamma} h_{E}^{\frac{1}{2}}\left\|\nabla \boldsymbol{u}_{h}\right\|_{0, \boldsymbol{T}_{E}}\left\|\boldsymbol{u}_{h}\right\|_{0, E} \leqslant \frac{1}{2}\left\|\boldsymbol{u}_{h}\right\|_{0, E}^{2}+c_{\Gamma}^{2} \frac{h_{E}}{2}\left\|\nabla \boldsymbol{u}_{h}\right\|_{0, \boldsymbol{T}_{E}}^{2} .
$$

Combining this inequality with 3.22 leads to

$$
\left\langle\mu_{\mathrm{eff}} \nabla \boldsymbol{w}_{h}^{\boldsymbol{u}_{h}} \cdot \boldsymbol{n}, \boldsymbol{u}_{h}\right\rangle_{E} \geqslant \frac{1}{2} \frac{h_{E}}{h_{E, \perp}}\left(\frac{\mu_{\mathrm{eff}}}{h_{E}}\left\|\boldsymbol{u}_{h}\right\|_{0, E}^{2}\right)-c_{\Gamma}^{2} \frac{h_{E}}{2 h_{E, \perp}}\left(\mu_{\mathrm{eff}}\left\|\nabla \boldsymbol{u}_{h}\right\|_{0, \boldsymbol{T}_{E}}^{2}\right) .
$$

The proof is concluded taking the sum over all boundary edges and defining

$$
c_{0}:=\frac{1}{2} \min _{E \in \mathcal{G}_{h}}\left\{\frac{h_{E}}{h_{E, \perp}}\right\} \quad c_{1}:=\frac{c_{\Gamma}^{2}}{2} \max _{E \in \mathcal{G}_{h}}\left\{\frac{h_{E}}{h_{E, \perp}}\right\}
$$

which are only dependent on the shape regularity of the mesh.

(2) First of all, since $\boldsymbol{w}_{h}^{\boldsymbol{u}_{h}}$ and $\boldsymbol{u}_{h}$ coincide on $E$, it holds $\left\|\boldsymbol{w}_{h}^{\boldsymbol{u}_{h}}\right\|_{0, E}^{2}=\left\|\boldsymbol{u}_{h}\right\|_{0, E}^{2}$. Moreover, let us consider a triangle $\boldsymbol{T}=\operatorname{conv}\left\{\boldsymbol{x}_{0}, \boldsymbol{x}_{1}, \boldsymbol{x}_{2}\right\}$ such that $\boldsymbol{T} \cap \Gamma \neq \emptyset$, assuming (without loss of generality) that $\boldsymbol{x}_{0} \notin \Gamma, \boldsymbol{x}_{1} \in \Gamma$ and denoting with $N \in\{1,2\}$ the number of vertices $\boldsymbol{T}$ has on the boundary. Using the linearity of $\boldsymbol{u}_{h}$, it holds, for an appropriate $c>0$ depending only on mesh regularity,

$$
\left\|\nabla \boldsymbol{w}_{h}^{\boldsymbol{u}_{h}}\right\|_{0, \boldsymbol{T}}^{2} \leqslant \begin{cases}c \sum_{i=1}^{N}\left|\boldsymbol{u}_{h}\left(\boldsymbol{x}_{i}\right)\right|^{2}, & \text { if } \boldsymbol{T} \cap \Gamma \neq \emptyset \\ 0, & \text { otherwise }\end{cases}
$$


Hence, denoting by $N_{\Gamma}$ the total number of boundary nodes, and by $c_{\mathrm{NB}}$ the maximum number of triangles adjacent to a boundary node (which can be bounded, e.g., depending on the smallest angle of the triangulation $\mathcal{T}_{h}$ ), one can write

$$
\mu_{\mathrm{eff}}\left\|\nabla \boldsymbol{w}_{h}^{\boldsymbol{u}_{h}}\right\|_{0}^{2} \leqslant \mu_{\mathrm{eff}} c_{\mathrm{NB}} c \sum_{i=1}^{N_{\Gamma}}\left|\boldsymbol{u}_{h}\left(\boldsymbol{x}_{i}\right)\right|^{2} \leqslant c_{2} \sum_{E \in \mathcal{G}_{h}} \frac{\mu_{\mathrm{eff}}}{h_{E}}\left\|\boldsymbol{u}_{h}\right\|_{E}^{2} .
$$

where $c_{2}$ depends only on the regularity of the mesh.

(3) The inequality $(3.19)$ can be proven using scaling arguments similar to the previous ones, observing that $\boldsymbol{w}_{h}^{\boldsymbol{u}_{h}}$ and $\boldsymbol{u}_{h}$ coincide on each boundary edge.

(4) Also the inequality (3.20) follows by standard scaling arguments, exploiting that $\boldsymbol{w}_{h}^{\boldsymbol{u}_{h}}$ is a component-wise linear function that vanishes on interior nodes of the mesh.

Remark 3.3 (Extension to higher order finite elements). It is worth noticing that an analogous of this Lemma can be also proven for higher order finite elements, using the same definition of the function $\boldsymbol{w}_{h}^{\boldsymbol{u}_{h}}$ with different definitions of the constants $c_{0}, c_{1}, c_{2}$, and $c_{3}$. In particular, some of the equalities (due to the fact that both $\boldsymbol{u}_{h}$ and $\boldsymbol{w}_{h}^{\boldsymbol{u}_{h}}$ are linear), e.g., (3.23) have to be replaced with inequalities obtained by proper scaling arguments.

Using the above defined function $\boldsymbol{w}_{h}^{\boldsymbol{u}_{h}}$, the next lemma allows to state stability of the boundary velocity.

Lemma 3.4 (Boundary control - I). Let $\alpha, \delta, \rho, \mu_{\mathrm{eff}}, \sigma \geqslant 0$ with $\mu_{\mathrm{eff}}+\sigma>0$. For any $\left(\boldsymbol{u}_{h}, p_{h}\right) \in \boldsymbol{V}_{h} \times Q_{h}$, there exist a function $\boldsymbol{w}_{h} \in \boldsymbol{V}_{h}$ and a constant $C_{2}=C_{2}(\alpha, \delta)>0$ which is independent from the physical parameters, from $\boldsymbol{u}_{h}$, and from $h$, such that

$$
A_{h}\left[\left(\boldsymbol{u}_{h}, p_{h}\right) ;\left(\boldsymbol{w}_{h}, 0\right)\right] \geqslant \frac{c_{0}}{4} \sum_{E \in \mathcal{G}_{h}} \theta \frac{\mu_{\mathrm{eff}}}{h_{E}}\left\|\boldsymbol{u}_{h}\right\|_{0, E}^{2}-C_{2}\left(\left\|\boldsymbol{u}_{h}\right\|^{2}+\alpha \sum_{\boldsymbol{T} \in \mathcal{T}_{h}} \frac{h_{\boldsymbol{T}}^{2}}{\nu}\left\|\nabla p_{h}\right\|_{0, \boldsymbol{T}}^{2}\right),
$$

where $c_{0}$ is the constant defined in Lemma 3.3.

Proof: For a given pair $\left(\boldsymbol{u}_{h}, p_{h}\right) \in \boldsymbol{V}_{h} \times Q_{h}$, let $\boldsymbol{w}_{h}:=\theta \boldsymbol{w}_{h}^{\boldsymbol{u}_{h}}$, where $\boldsymbol{w}_{h}^{\boldsymbol{u}_{h}}$ is the function defined in Lemma 3.3 . Then, we get

$$
\begin{aligned}
A_{h}\left[\left(\boldsymbol{u}_{h}, p_{h}\right) ;\left(\boldsymbol{w}_{h}, 0\right)\right]= & \theta \mu_{\mathrm{eff}}\left(\nabla \boldsymbol{u}_{h}, \nabla \boldsymbol{w}_{h}^{\boldsymbol{u}_{h}}\right)+\theta\left(\sigma \boldsymbol{u}_{h}, \boldsymbol{w}_{h}^{\boldsymbol{u}_{h}}\right)-\theta\left\langle\mu_{\mathrm{eff}} \nabla \boldsymbol{u}_{h} \cdot \boldsymbol{n}, \boldsymbol{w}_{h}^{\boldsymbol{u}_{h}}\right\rangle+\theta\left\langle\mu_{\mathrm{eff}} \nabla \boldsymbol{w}_{h}^{\boldsymbol{u}_{h}} \cdot \boldsymbol{n}, \boldsymbol{u}_{h}\right\rangle \\
& -\theta\left(p_{h}, \nabla \cdot \boldsymbol{w}_{h}^{\boldsymbol{u}_{h}}\right)+\theta\left\langle p_{h} \boldsymbol{n}, \boldsymbol{w}_{h}^{\boldsymbol{u}_{h}}\right\rangle+\alpha \theta \sum_{\boldsymbol{T} \in \mathcal{T}_{h}} \frac{h_{\boldsymbol{T}}^{2}}{\nu}\left(\sigma \boldsymbol{u}_{h}+\nabla p_{h}, \sigma \boldsymbol{w}_{h}^{\boldsymbol{u}_{h}}\right)_{\boldsymbol{T}} \\
& +\delta \nu \theta\left(\nabla \cdot \boldsymbol{u}_{h}, \nabla \cdot \boldsymbol{w}_{h}^{\boldsymbol{u}_{h}}\right)+\rho \nu \theta \sum_{\boldsymbol{x} \in \mathcal{C}}\left|\llbracket \boldsymbol{u}_{h} \cdot \boldsymbol{n} \rrbracket(\boldsymbol{x})\right|^{2}
\end{aligned}
$$

Observing that the corner stabilization is always positive and that $\theta \leqslant 1$, using the Cauchy-Schwarz inequality, the inequalities (3.17), (3.18), and (3.19) leads to

$$
\begin{aligned}
A_{h}\left[\left(\boldsymbol{u}_{h}, p_{h}\right) ;\left(\boldsymbol{w}_{h}, 0\right)\right] \geqslant & -c_{2}^{\frac{1}{2}} \mu_{\mathrm{eff}}^{\frac{1}{2}}\left\|\nabla \boldsymbol{u}_{h}\right\|_{0} \underbrace{\theta^{\frac{1}{2}}}_{\leqslant 1}\left(\sum_{E \in \mathcal{G}_{h}} \theta \frac{\mu_{\mathrm{eff}}}{h_{E}}\left\|\boldsymbol{u}_{h}\right\|_{0, E}^{2}\right)^{\frac{1}{2}}-\sigma c_{3}\left\|\boldsymbol{u}_{h}\right\|_{0}^{2} \\
& -\theta\left\langle\mu_{\mathrm{eff}} \nabla \boldsymbol{u}_{h} \cdot \boldsymbol{n}, \boldsymbol{w}_{h}^{\boldsymbol{u}_{h}}\right\rangle+c_{0} \sum_{E \in \mathcal{G}_{h}} \theta \frac{\mu_{\mathrm{eff}}}{h_{E}}\left\|\boldsymbol{u}_{h}\right\|_{0, E}^{2}-c_{1} \mu_{\mathrm{eff}}\left\|\nabla \boldsymbol{u}_{h}\right\|_{0}^{2} \\
& -\theta\left(p_{h}, \nabla \cdot \boldsymbol{w}_{h}^{\boldsymbol{u}_{h}}\right)+\theta\left\langle p_{h} \boldsymbol{n}, \boldsymbol{w}_{h}^{\boldsymbol{u}_{h}}\right\rangle+\alpha \theta \sum_{\boldsymbol{T} \in \mathcal{T}_{h}} \frac{h_{\boldsymbol{T}}^{2}}{\nu}\left(\sigma \boldsymbol{u}_{h}+\nabla p_{h}, \sigma \boldsymbol{w}_{h}^{\boldsymbol{u}_{h}}\right)_{\boldsymbol{T}} \\
& +\delta \nu \theta\left(\nabla \cdot \boldsymbol{u}_{h}, \nabla \cdot \boldsymbol{w}_{h}^{\boldsymbol{u}_{h}}\right) .
\end{aligned}
$$


Combining the Cauchy-Schwarz inequality, the trace inequality $3.3 \mathrm{~b}$, and the fact $\left\|\boldsymbol{w}_{h}^{\boldsymbol{u}_{h}}\right\|_{0, E}=\left\|\boldsymbol{u}_{h}\right\|_{0, E}$ we obtain

$$
\theta\left\langle\mu_{\mathrm{eff}} \nabla \boldsymbol{u}_{h} \cdot \boldsymbol{n}, \boldsymbol{w}_{h}^{\boldsymbol{u}_{h}}\right\rangle \leqslant\left(c_{\mathrm{DTI}} \mu_{\mathrm{eff}}\right)^{\frac{1}{2}}\left\|\nabla \boldsymbol{u}_{h}\right\|_{0} \underbrace{\theta^{\frac{1}{2}}}_{\leqslant 1}\left(\sum_{E \in \mathcal{G}_{h}} \theta \frac{\mu_{\mathrm{eff}}}{h_{E}}\left\|\boldsymbol{u}_{h}\right\|_{0, E}^{2}\right)^{\frac{1}{2}},
$$

which, inserted in (3.26), yields

$$
\begin{aligned}
A_{h}\left[\left(\boldsymbol{u}_{h}, p_{h}\right) ;\left(\boldsymbol{w}_{h}, 0\right)\right] \geqslant & -\left(c_{2}^{\frac{1}{2}}+c_{\mathrm{DTI}}^{\frac{1}{2}}\right) \mu_{\mathrm{eff}}^{\frac{1}{2}}\left\|\nabla \boldsymbol{u}_{h}\right\|_{0}\left(\sum_{E \in \mathcal{G}_{h}} \theta \frac{\mu_{\mathrm{eff}}}{h_{E}}\left\|\boldsymbol{u}_{h}\right\|_{0, E}^{2}\right)^{\frac{1}{2}}-\sigma c_{3}\left\|\boldsymbol{u}_{h}\right\|_{0}^{2} \\
& +c_{0} \sum_{E \in \mathcal{G}_{h}} \theta \frac{\mu_{\mathrm{eff}}}{h_{E}}\left\|\boldsymbol{u}_{h}\right\|_{0, E}^{2}-c_{1} \mu_{\mathrm{eff}}\left\|\nabla \boldsymbol{u}_{h}\right\|_{0}^{2} \\
& \underbrace{-\theta\left(p_{h}, \nabla \cdot \boldsymbol{w}_{h}^{\boldsymbol{u}_{h}}\right)+\theta\left\langle p_{h} \boldsymbol{n}, \boldsymbol{w}_{h}^{\left.\boldsymbol{u}_{h}\right\rangle}\right\rangle}_{=: \mathcal{Q}_{1}}+\underbrace{\alpha \theta \sum_{\boldsymbol{T} \in \mathcal{T}_{h}} \frac{h_{\boldsymbol{T}}^{2}}{\nu}\left(\sigma \boldsymbol{u}_{h}+\nabla p_{h}, \sigma \boldsymbol{w}_{h}^{\boldsymbol{u}_{h}}\right)_{\boldsymbol{T}}}_{=: \mathcal{Q}_{2}} \\
& +\underbrace{\delta \nu \theta\left(\nabla \cdot \boldsymbol{u}_{h}, \nabla \cdot \boldsymbol{w}_{h}^{\left.\boldsymbol{u}_{h}\right)}\right.}_{=: \mathcal{Q}_{3}} .
\end{aligned}
$$

In order to bound the term $\mathcal{Q}_{1}$, we use the integration by parts formula, the Cauchy-Schwarz inequality, the inequalities (3.20), 3.18), and $\theta \nu=\mu_{\mathrm{eff}}$ to obtain

$$
\begin{aligned}
\mathcal{Q}_{1} & =\sum_{\boldsymbol{T} \in \mathcal{T}_{h}} \theta\left(\nabla p_{h}, \boldsymbol{w}_{h}^{\boldsymbol{u}_{h}}\right)_{\boldsymbol{T}} \leqslant \theta\left(\alpha \sum_{\boldsymbol{T} \in \mathcal{T}_{h}} \frac{h_{\boldsymbol{T}}^{2}}{\nu}\left\|\nabla p_{h}\right\|_{0, \boldsymbol{T}}^{2}\right)^{\frac{1}{2}}\left(\sum_{\boldsymbol{T} \in \mathcal{T}_{h}} \frac{\nu}{\alpha h_{\boldsymbol{T}}^{2}}\left\|\boldsymbol{w}_{h}^{\boldsymbol{u}_{h}}\right\|_{0, \boldsymbol{T}}^{2}\right)^{\frac{1}{2}} \\
& \leqslant\left(\alpha \sum_{\boldsymbol{T} \in \mathcal{T}_{h}} \frac{h_{\boldsymbol{T}}^{2}}{\nu}\left\|\nabla p_{h}\right\|_{0, \boldsymbol{T}}^{2}\right)^{\frac{1}{2}}\left(\theta^{2} \nu \widetilde{c_{3}} \frac{1}{\alpha}\left\|\nabla \boldsymbol{w}_{h}^{\boldsymbol{u}_{h}}\right\|_{0}^{2}\right)^{\frac{1}{2}} \\
& \leqslant\left(\frac{c_{2} \widetilde{c_{3}}}{\alpha}\right)^{\frac{1}{2}}\left(\alpha \sum_{\boldsymbol{T} \in \mathcal{T}_{h}} \frac{h_{\boldsymbol{T}}^{2}}{\nu}\left\|\nabla p_{h}\right\|_{0, \boldsymbol{T}}^{2}\right)^{\frac{1}{2}}\left(\sum_{E \in \mathcal{G}_{h}} \theta \frac{\mu_{\mathrm{eff}}}{h_{E}}\left\|\boldsymbol{u}_{h}\right\|_{0, E}^{2}\right)^{\frac{1}{2}} .
\end{aligned}
$$

Next, we observe that the term $\mathcal{Q}_{2}$, coming from the pressure stabilization, can be bounded using the CauchySchwarz inequality, Young's inequality, and (3.19) as

$$
\begin{aligned}
\mathcal{Q}_{2} & \geqslant-\theta \alpha^{\frac{1}{2}}\left(\alpha \sum_{\boldsymbol{T} \in \mathcal{T}_{h}} \frac{h_{\boldsymbol{T}}^{2}}{\nu}\left\|\nabla p_{h}\right\|_{0, \boldsymbol{T}}^{2}\right)^{\frac{1}{2}}(\sum_{\boldsymbol{T} \in \mathcal{T}_{h}} \underbrace{\frac{\sigma h_{\boldsymbol{T}}^{2}}{\nu}}_{\leqslant 1} \sigma\left\|\boldsymbol{w}_{h}^{\boldsymbol{u}_{h}}\right\|_{0, \boldsymbol{T}}^{2})^{\frac{1}{2}}-\theta \alpha \sigma c_{3}\left\|\boldsymbol{u}_{h}\right\|_{0}^{2} \\
& \geqslant-\left(\alpha \sum_{\boldsymbol{T} \in \mathcal{T}_{h}} \frac{h_{\boldsymbol{T}}^{2}}{\nu}\left\|\nabla p_{h}\right\|_{0, \boldsymbol{T}}^{2}\right)^{\frac{1}{2}}(\alpha \sigma)^{\frac{1}{2}} c_{3}\left\|\boldsymbol{u}_{h}\right\|_{0}-\alpha \sigma c_{3}\left\|\boldsymbol{u}_{h}\right\|_{0}^{2} \\
& \geqslant-\frac{1}{4} \alpha \sum_{\boldsymbol{T} \in \mathcal{T}_{h}} \frac{h_{\boldsymbol{T}}^{2}}{\nu}\left\|\nabla p_{h}\right\|_{0, \boldsymbol{T}}^{2}-\left(c_{3}^{2}+c_{3}\right) \alpha \sigma\left\|\boldsymbol{u}_{h}\right\|_{0}^{2} .
\end{aligned}
$$


Finally, 3.18 and $(\nu \theta)^{\frac{1}{2}}=\mu_{\text {eff }}^{\frac{1}{2}}$ allow also to conclude

$$
\mathcal{Q}_{3} \geqslant-\delta(\nu \theta n)^{\frac{1}{2}}\left\|\nabla \cdot \boldsymbol{u}_{h}\right\|_{0}(\nu \theta)^{\frac{1}{2}}\left\|\nabla \boldsymbol{w}_{h}^{\boldsymbol{u}_{h}}\right\|_{0} \geqslant-\left(\delta n c_{2}\right)^{\frac{1}{2}}\left(\delta \nu\left\|\nabla \cdot \boldsymbol{u}_{h}\right\|_{0}^{2}\right)^{\frac{1}{2}}\left(\sum_{E \in \mathcal{G}_{h}} \theta \frac{\mu_{\mathrm{eff}}}{h_{E}}\left\|\boldsymbol{u}_{h}\right\|_{0, E}^{2}\right)^{\frac{1}{2}} .
$$

We observe that scaling the test function by $\theta$ allows to, on the one hand, assure coercivity in the chosen norm, and, on the other hand, to obtain a parameter independent estimate for the terms involving $\nu \nabla \cdot \boldsymbol{w}_{h}$. Notice as well that the scaling by $\theta$ implies that the test function vanishes in the Darcy limit $\left(\mu_{\mathrm{eff}}=0\right)$. Inserting $(3.28$, , (3.29), and 3.30 into (3.27), and reordering the terms yields

$$
\begin{aligned}
A_{h}\left[\left(\boldsymbol{u}_{h}, p_{h}\right) ;\left(\boldsymbol{w}_{h}, 0\right)\right] \geqslant & c_{0} \sum_{E \in \mathcal{G}_{h}} \theta \frac{\mu_{\mathrm{eff}}}{h_{E}}\left\|\boldsymbol{u}_{h}\right\|_{0, E}^{2}-c_{1} \mu_{\mathrm{eff}}\left\|\nabla \boldsymbol{u}_{h}\right\|_{0}^{2}-\left(c_{3}+\alpha\left(c_{3}^{2}+c_{3}\right)\right) \sigma\left\|\boldsymbol{u}_{h}\right\|_{0}^{2} \\
& -\left(c_{2}^{\frac{1}{2}}+c_{\mathrm{DTI}}^{\frac{1}{2}}\right) \mu_{\mathrm{eff}}^{\frac{1}{2}}\left\|\nabla \boldsymbol{u}_{h}\right\|_{0}\left(\sum_{E \in \mathcal{G}_{h}} \theta \frac{\mu_{\mathrm{eff}}}{h_{E}}\left\|\boldsymbol{u}_{h}\right\|_{0, E}^{2}\right)^{\frac{1}{2}}-\frac{1}{4} \alpha \sum_{\boldsymbol{T} \in \mathcal{T}_{h}} \frac{h_{\boldsymbol{T}}^{2}}{\nu}\left\|\nabla p_{h}\right\|_{0, \boldsymbol{T}}^{2} \\
& -\left(\frac{c_{2} \widetilde{c_{3}}}{\alpha}\right)^{\frac{1}{2}}\left(\alpha \sum_{\boldsymbol{T}} \frac{h_{\boldsymbol{T}}^{2}}{\nu}\left\|\nabla p_{h}\right\|_{0, \boldsymbol{T}}^{2}\right)^{\frac{1}{2}}\left(\sum_{E} \theta \frac{\nu}{h_{E}}\left\|\boldsymbol{u}_{h}\right\|_{0, E}^{2}\right)^{\frac{1}{2}} \\
& -\left(\delta n c_{2}\right)^{\frac{1}{2}}\left(\delta \nu\left\|\nabla \cdot \boldsymbol{u}_{h}\right\|_{0}^{2}\right)^{\frac{1}{2}}\left(\sum_{E \in \mathcal{G}_{h}} \theta \frac{\mu_{\mathrm{eff}}}{h_{E}}\left\|\boldsymbol{u}_{h}\right\|_{0, E}^{2}\right)^{\frac{1}{2}} \cdot
\end{aligned}
$$

Applying three times the Young inequality yields, for any $\varepsilon>0$,

$$
\begin{aligned}
A_{h}\left[\left(\boldsymbol{u}_{h}, p_{h}\right) ;\left(\boldsymbol{w}_{h}, 0\right)\right] \geqslant & c_{0} \sum_{E \in \mathcal{G}_{h}} \theta \frac{\mu_{\mathrm{eff}}}{h_{E}}\left\|\boldsymbol{u}_{h}\right\|_{0, E}^{2}-c_{1} \mu_{\mathrm{eff}}\left\|\nabla \boldsymbol{u}_{h}\right\|_{0}^{2}-\left(c_{3}+\alpha\left(c_{3}^{2}+c_{3}\right)\right) \sigma\left\|\boldsymbol{u}_{h}\right\|_{0}^{2} \\
& -\frac{\left(c_{2}^{\frac{1}{2}}+c_{\mathrm{DTI}}^{\frac{1}{2}}\right)^{2}}{2 \varepsilon} \mu_{\mathrm{eff}}\left\|\nabla \boldsymbol{u}_{h}\right\|_{0}^{2}-\frac{\varepsilon}{2} \sum_{E \in \mathcal{G}_{h}} \theta \frac{\mu_{\mathrm{eff}}}{h_{E}}\left\|\boldsymbol{u}_{h}\right\|_{0, E}^{2}-\frac{1}{4} \alpha \sum_{\boldsymbol{T} \in \mathcal{T}_{h}} \frac{h_{\boldsymbol{T}}^{2}}{\nu}\left\|\nabla p_{h}\right\|_{0, \boldsymbol{T}}^{2} \\
& -\frac{c_{2} \widetilde{c_{3}}}{2 \alpha \varepsilon}\left(\alpha \sum_{\boldsymbol{T}} \frac{h_{\boldsymbol{T}}^{2}}{\nu}\left\|\nabla p_{h}\right\|_{0, \boldsymbol{T}}^{2}\right)-\frac{\varepsilon}{2}\left(\sum_{E} \theta \frac{\mu_{\mathrm{eff}}}{h_{E}}\left\|\boldsymbol{u}_{h}\right\|_{0, E}^{2}\right) \\
& -\frac{\delta n c_{2}}{2 \varepsilon}\left(\delta \nu\left\|\nabla \cdot \boldsymbol{u}_{h}\right\|_{0}^{2}\right)-\frac{\varepsilon}{2}\left(\sum_{E \in \mathcal{G}_{h}} \theta \frac{\mu_{\mathrm{eff}}}{h_{E}}\left\|\boldsymbol{u}_{h}\right\|_{0, E}^{2}\right) .
\end{aligned}
$$

Choosing $\varepsilon=\frac{c_{0}}{2}$ allows to conclude

$$
\begin{aligned}
A_{h}\left[\left(\boldsymbol{u}_{h}, p_{h}\right) ;\left(\boldsymbol{w}_{h}, 0\right)\right] \geqslant & \frac{c_{0}}{4} \sum_{E \in \mathcal{G}_{h}} \theta \frac{\mu_{\mathrm{eff}}}{h_{E}}\left\|\boldsymbol{u}_{h}\right\|_{0, E}^{2}-\left(c_{1}+\frac{\left(c_{2}^{\frac{1}{2}}+c_{\mathrm{DTI}}^{\frac{1}{2}}\right)^{2}}{c_{0}}\right) \mu_{\mathrm{eff}}\left\|\nabla \boldsymbol{u}_{h}\right\|_{0}^{2} \\
& -\left(c_{3}+\alpha\left(c_{3}^{2}+c_{3}\right)\right) \sigma\left\|\boldsymbol{u}_{h}\right\|_{0}^{2}-\left(\frac{1}{4}+\frac{c_{2} \widetilde{c_{3}}}{\alpha c_{0}}\right) \alpha \sum_{\boldsymbol{T} \in \mathcal{T}_{h}} \frac{h_{\boldsymbol{T}}^{2}}{\nu}\left\|\nabla p_{h}\right\|_{0, \boldsymbol{T}}^{2} \\
& -\frac{\delta n c_{2}}{c_{0}}\left(\delta \nu\left\|\nabla \cdot \boldsymbol{u}_{h}\right\|_{0}^{2}\right) .
\end{aligned}
$$


The proof is completed defining

$$
C_{2}:=\max \left\{c_{1}+\frac{\left(c_{2}^{\frac{1}{2}}+c_{\mathrm{DTI}}^{\frac{1}{2}}\right)^{2}}{c_{0}},\left(c_{3}+\alpha\left(c_{3}^{2}+c_{3}\right)\right), \frac{1}{4}+\frac{c_{2} \widetilde{c_{3}}}{\alpha c_{0}}, \frac{\delta n c_{2}}{c_{0}}\right\} .
$$

Remark 3.4 (On the behavior of $C_{2}$ ). It is worth noticing that the constant $C_{2}$ is bounded for any choice of the stabilization parameters. In particular, $C_{2}$ grows like $c_{0}^{-1}$, which is related to the anisotropy of the mesh near the boundary (see (3.25)). Moreover, as it has been stated in Remark 2.3, the stability estimate does not rely on the particular technique chosen for the stabilization of the equal-order finite element (i.e., $\alpha>0$ is not strictly required).

The last step needed to show the fulfillment of the inf-sup condition is related to the control of the normal velocity at the boundary, which is particularly relevant in order to guarantee stability towards the Darcy limit, i.e., for $\sigma \ell_{\Omega}^{2} \gg \mu_{\mathrm{eff}}$ and especially $\mu_{\mathrm{eff}}=0$.

It is worth recalling our assumption (2.4) on the mesh, stating that for any two adjacent boundary edges $E, E^{\prime} \in \mathcal{G}_{h}$ it holds

$$
\frac{h_{E}}{h_{E^{\prime}}} \leqslant \eta_{0}<7+4 \sqrt{3}(\approx 13.9) .
$$

We observe that this assumption is weaker than quasi-uniformity of the mesh, as it only restricts the ratio between the lengths of adjacent boundary edges.

Moreover, let us also recall that the mesh is assumed to satisfy (2.5), i.e., that the area of inner triangles is larger than the area of the boundary triangles.

Lemma 3.5. Let us assume that the family of triangulations $\left\{\mathcal{T}_{h}\right\}_{h}$ satisfies (3.31) and (2.5). For a given $\boldsymbol{u}_{h} \in \boldsymbol{V}_{h}$, let us define $q_{h}^{\boldsymbol{u}_{h}} \in Q_{h}$ as the function whose values at the boundary nodes are uniquely defined to satisfy the $L^{2}$-projection property

$$
\left\langle q_{h}^{\boldsymbol{u}_{h}}, \varphi_{h}\right\rangle=-\sum_{E \in \mathcal{G}_{h}} \frac{1}{h_{E}}\left\langle\boldsymbol{u}_{h} \cdot \boldsymbol{n}_{E}, \varphi_{h}\right\rangle_{E}, \quad \forall \varphi_{h} \in Q_{h} .
$$

and its value at the interior nodes is given by a constant $c_{q}$, chosen in order to satisfy $\int_{\boldsymbol{\Omega}} q_{h}^{\boldsymbol{u}_{h}}=0$. Then the function $q_{h}^{\boldsymbol{u}_{h}}$ has the following properties:

(1) There exists a constant $c_{4}>0$, depending only on $\eta_{0}$, such that

$$
\sum_{E \in \mathcal{G}_{h}} h_{E}\left\|q_{h}^{\boldsymbol{u}_{h}}\right\|_{0, E}^{2} \leqslant c_{4} \sum_{E \in \mathcal{G}_{h}} \frac{1}{h_{E}}\left\|\boldsymbol{u}_{h} \cdot \boldsymbol{n}_{E}\right\|_{0, E}^{2}
$$

(2) There exists a constant $c_{5}>0$, depending only on $\eta_{0}$, such that

$$
-\left\langle q_{h}^{\boldsymbol{u}_{h}}, \boldsymbol{u}_{h} \cdot \boldsymbol{n}\right\rangle \geqslant \frac{1}{2} \sum_{E \in \mathcal{G}_{h}} \frac{1}{h_{E}}\left\|\boldsymbol{u}_{h} \cdot \boldsymbol{n}_{E}\right\|_{0, E}^{2}-c_{5} \sum_{\boldsymbol{x} \in \mathcal{C}}\left|\llbracket \boldsymbol{u}_{h} \cdot \boldsymbol{n} \rrbracket(\boldsymbol{x})\right|^{2} .
$$

(3) There exists a constant $c_{6}>0$, depending only on the properties of the mesh, such that

$$
\left\|q_{h}^{\boldsymbol{u}_{h}}\right\|_{0}^{2} \leqslant c_{6} \sum_{E \in \mathcal{G}_{h}} \frac{1}{h_{E}}\left\|\boldsymbol{u}_{h} \cdot \boldsymbol{n}_{E}\right\|_{0, E}^{2}
$$


and

$$
\sum_{\boldsymbol{T} \in \mathcal{T}_{h}} h_{\boldsymbol{T}}^{2}\left\|\nabla q_{h}^{\boldsymbol{u}_{h}}\right\|_{0, \boldsymbol{T}}^{2} \leqslant c_{6} c_{I}^{2} \sum_{E \in \mathcal{G}_{h}} \frac{1}{h_{E}}\left\|\boldsymbol{u}_{h} \cdot \boldsymbol{n}_{E}\right\|_{0, E}^{2}
$$

Proof:

(1) In order to prove (3.33), let us restrict for simplicity, and without loss of generality, to the case of a boundary with a single connected component. In this case, let us number the boundary nodes as $\boldsymbol{x}_{1}, \ldots, \boldsymbol{x}_{N}$ and the boundary edges as $E_{1}, \ldots, E_{N}$ such that the edge $E_{i}$ connects the nodes $\boldsymbol{x}_{i}$ and $\boldsymbol{x}_{i+1}$, for all $i \in\{1, \ldots, N\}$. Moreover, we identify $\boldsymbol{x}_{N+1}$ with $\boldsymbol{x}_{1}$, so that the above defined convention is well-defined also for $i=N$. To simplify the notations, let us abbreviate $h_{i}=h_{E_{i}}$ and $q_{i}=q_{h}^{\boldsymbol{u}_{h}}\left(\boldsymbol{x}_{i}\right)$. We now consider a function $\varphi_{h} \in Q_{h}$ defined at each node $\boldsymbol{x}$ of the mesh by

$$
\varphi_{h}(\boldsymbol{x}):= \begin{cases}h_{i} q_{i}, & \boldsymbol{x} \in \Gamma \\ c_{\varphi}, & \text { otherwise }\end{cases}
$$

where $c_{\varphi}$ is a constant defined in order to have $\int_{\Omega} \varphi=0$. On any boundary edge $E_{i}$, by the linearity of $q_{h}^{\boldsymbol{u}_{h}}$ and $\varphi_{h}$, application of the Simpson rule yields

$$
\int_{E_{i}} q_{h}^{\boldsymbol{u}_{h}} \varphi_{h}=\frac{h_{i}}{6}\left(2 h_{i} q_{i}^{2}+\left(h_{i}+h_{i+1}\right) q_{i} q_{i+1}+2 h_{i+1} q_{i+1}^{2}\right)
$$

and

$$
\int_{E_{i}}\left|q_{h}^{\boldsymbol{u}_{h}}\right|^{2}=\frac{h_{i}}{3}\left(q_{i}^{2}+q_{i} q_{i+1}+q_{i+1}^{2}\right) .
$$

We will first prove that there exists a constant $\hat{c}>0$, independent from $q_{h}^{\boldsymbol{u}_{h}}$ and $h_{i}$, such that

$$
h_{i} \int_{E_{i}}\left|q_{h}^{\boldsymbol{u}_{h}}\right|^{2} \leqslant \hat{c} \int_{E_{i}} q_{h}^{\boldsymbol{u}_{h}} \varphi_{h} .
$$

If $q_{i}=0$, 3.37 holds if $\frac{h_{i}}{h_{i+1}} \leqslant \hat{c}$, i.e., with $\hat{c} \geqslant \eta_{0}$. Assume now that $q_{i} \neq 0$ and set $\eta:=\frac{h_{i+1}}{h_{i}}$ and $t:=\frac{q_{i+1}}{q_{i}}$. The inequality (3.37) then reduces to

$$
1+t+t^{2} \leqslant \frac{\hat{c}}{2}\left(2+(1+\eta) t+2 \eta t^{2}\right) .
$$

Since $\eta>0, \eta \in\left[1 / \eta_{0}, \eta_{0}\right]$, and $1+t+t^{2}>0$, for all $t \in \mathbb{R}$, the above condition is equivalent to

$$
\inf _{\frac{1}{\eta_{0}} \leqslant \eta \leqslant \eta_{0}} \frac{2+(1+\eta) t+2 \eta t^{2}}{1+t+t^{2}}>0 .
$$

Since the polynomial in the denominator is always strictly positive, the whole infimum is positive if the numerator as a polynomial in $t$ is strictly positive for all $t \in \mathbb{R}$ and for all $\eta \in\left[1 / \eta_{0}, \eta_{0}\right]$. This is the case if and only if its discriminant does not have real roots for the selected range of $\eta$. The discriminant of this polynomial is $\mathbb{D}(\eta):=(1+\eta)^{2}-16 \eta$, which vanishes if $\eta=7 \pm 4 \sqrt{3}$ and is strictly negative if $\eta \in(7-4 \sqrt{3}, 7+4 \sqrt{3})=$ $\left(\frac{1}{7+4 \sqrt{3}}, 7+4 \sqrt{3}\right)$. Hence, $\mathbb{D}(\eta)$ is negative if $\eta_{0}<7+4 \sqrt{3}$ (i.e., assumption (3.31). Estimate (3.37) is therefore proven by setting

$$
\hat{c}=\hat{c}\left(\eta_{0}\right):=\max \left\{\eta_{0}, 2\left(\inf _{\substack{\frac{1}{\eta_{0}} \leqslant \eta \leqslant \eta_{0} \\ t \in \mathbb{R}}} \frac{2+(1+\eta) t+2 \eta t^{2}}{1+t+t^{2}}\right)^{-1}\right\} .
$$


The assumption $\frac{h_{i+1}}{h_{i}} \leqslant \eta_{0}$ yields also, on any boundary edge $E_{i}$

$$
\frac{1}{h_{i}}\left\|\varphi_{h}\right\|_{0, E_{i}}^{2} \leqslant h_{i}\left(1+\frac{h_{i+1}}{h_{i}}+\left(\frac{h_{i+1}}{h_{i}}\right)^{2}\right)\left\|q_{h}^{\boldsymbol{u}_{h}}\right\|_{0, E_{i}}^{2} \leqslant\left(1+\eta_{0}+\eta_{0}^{2}\right) h_{i}\left\|q_{h}^{\boldsymbol{u}_{h}}\right\|_{0, E_{i}}^{2} .
$$

Summing 3.37) over all boundary edges and using (3.32), the Cauchy-Schwarz inequality, (3.38), and the Young inequality yields

$$
\begin{aligned}
\sum_{E \in \mathcal{G}_{h}} h_{E}\left\|q_{h}^{\boldsymbol{u}_{h}}\right\|_{0, E}^{2} \leqslant \hat{c}\left\langle q_{h}^{\boldsymbol{u}_{h}}, \varphi_{h}\right\rangle & =-\hat{c} \sum_{E \in \mathcal{G}_{h}} \frac{1}{h_{E}}\left\langle\boldsymbol{u}_{h} \cdot \boldsymbol{n}_{E}, \varphi_{h}\right\rangle_{E} \\
& \leqslant \hat{c}\left(\sum_{E \in \mathcal{G}_{h}} \frac{1}{h_{E}}\left\|\boldsymbol{u}_{h} \cdot \boldsymbol{n}_{E}\right\|_{0, E}^{2}\right)^{\frac{1}{2}}\left(\sum_{E \in \mathcal{G}_{h}} \frac{1}{h_{E}}\left\|\varphi_{h}\right\|_{0, E}^{2}\right)^{\frac{1}{2}} \\
& \leqslant \hat{c}\left(\frac{\varepsilon}{2} \sum_{E \in \mathcal{G}_{h}} \frac{1}{h_{E}}\left\|\boldsymbol{u}_{h} \cdot \boldsymbol{n}_{E}\right\|_{0, E}^{2}+\frac{1}{2 \varepsilon}\left(1+\eta_{0}+\eta_{0}^{2}\right) \sum_{E \in \mathcal{G}_{h}} h_{E}\left\|q_{h}^{\boldsymbol{u}_{h}}\right\|_{0, E}^{2}\right)
\end{aligned}
$$

Choosing $\varepsilon:=\hat{c}\left(1+\eta_{0}+\eta_{0}^{2}\right)$ leads to

$$
\sum_{E \in \mathcal{G}_{h}} h_{E}\left\|q_{h}^{\boldsymbol{u}_{h}}\right\|_{0, E}^{2} \leqslant \hat{c}^{2}\left(1+\eta_{0}+\eta_{0}^{2}\right) \sum_{E \in \mathcal{G}_{h}} \frac{1}{h_{E}}\left\|\boldsymbol{u}_{h} \cdot \boldsymbol{n}_{E}\right\|_{0, E}^{2}
$$

Estimate 3.33 is obtained defining $c_{4}:=\hat{c}^{2}\left(1+\eta_{0}+\eta_{0}^{2}\right)$, which only depends on $\eta_{0}$.

(2) To prove the second inequality, let us consider the function $\varphi_{h} \in Q_{h}$ such that, at the mesh nodes $\boldsymbol{x}$, it holds

$$
\varphi_{h}(\boldsymbol{x}):= \begin{cases}\boldsymbol{u}_{h} \cdot \boldsymbol{n}(\boldsymbol{x}), & \text { for } \boldsymbol{x} \in \Gamma \backslash \mathcal{C} \\ \frac{1}{2}\left(\boldsymbol{u}_{h} \cdot \boldsymbol{n}_{E}(\boldsymbol{x})+\boldsymbol{u}_{h} \cdot \boldsymbol{n}_{E^{\prime}}(\boldsymbol{x})\right), & \text { for } \boldsymbol{x} \in \mathcal{C}, \text { with } E, E^{\prime} \text { two adjacent boundary edges } \\ c_{\varphi}, & \text { otherwise }\end{cases}
$$

with a constant $c_{\varphi}$ defined in order to have $\int_{\Omega} \varphi_{h}=0$. Remember that $\mathcal{C}$ is the set of corner nodes at the boundary.

Using 3.32, the Cauchy-Schwarz, and the Young inequalities we obtain, for any $\varepsilon>0$,

$$
\begin{aligned}
-\left\langle q_{h}^{\boldsymbol{u}_{h}}, \boldsymbol{u}_{h} \cdot \boldsymbol{n}\right\rangle & =-\left\langle q_{h}^{\boldsymbol{u}_{h}}, \varphi_{h}\right\rangle-\left\langle q_{h}^{\boldsymbol{u}_{h}}, \boldsymbol{u}_{h} \cdot \boldsymbol{n}-\varphi_{h}\right\rangle=\sum_{E \in \mathcal{G}_{h}} \frac{1}{h_{E}}\left\langle\boldsymbol{u}_{h} \cdot \boldsymbol{n}_{E}, \varphi_{h}\right\rangle_{E}-\sum_{E \in \mathcal{G}_{h}}\left\langle q_{h}^{\boldsymbol{u}_{h}}, \boldsymbol{u}_{h} \cdot \boldsymbol{n}_{E}-\varphi_{h}\right\rangle_{E} \\
& =\sum_{E \in \mathcal{G}_{h}} \frac{1}{h_{E}}\left\|\boldsymbol{u}_{h} \cdot \boldsymbol{n}_{E}\right\|_{0, E}^{2}-\sum_{E \in \mathcal{G}_{h}} \frac{1}{h_{E}}\left\langle\boldsymbol{u}_{h} \cdot \boldsymbol{n}_{E}, \boldsymbol{u}_{h} \cdot \boldsymbol{n}_{E}-\varphi_{h}\right\rangle_{E}-\sum_{E \in \mathcal{G}_{h}}\left\langle q_{h}^{\boldsymbol{u}_{h}}, \boldsymbol{u}_{h} \cdot \boldsymbol{n}_{E}-\varphi_{h}\right\rangle_{E} \\
& \geqslant \sum_{E \in \mathcal{G}_{h}} \frac{1}{h_{E}}\left(\left(1-\frac{\varepsilon}{2}\right)\left\|\boldsymbol{u}_{h} \cdot \boldsymbol{n}_{E}\right\|_{0, E}^{2}-\frac{1}{\varepsilon}\left\|\boldsymbol{u}_{h} \cdot \boldsymbol{n}_{E}-\varphi_{h}\right\|_{0, E}^{2}\right)-\frac{\varepsilon}{2} \sum_{E \in \mathcal{G}_{h}} h_{E}\left\|q_{h}^{\boldsymbol{u}_{h}}\right\|_{0, E}^{2} \cdot(3.39)
\end{aligned}
$$

The function $\left.\left(\boldsymbol{u}_{h} \cdot \boldsymbol{n}-\varphi_{h}\right)\right|_{E \in \mathcal{G}_{h}}$ is different from zero only on boundary edges that are adjacent to a corner. In particular, let us consider a corner node $\boldsymbol{x}_{c} \in \mathcal{C}$ with an adjacent edge $E=\overline{\boldsymbol{x}_{i} \boldsymbol{x}_{c}}$. It holds

$$
\left|\left(\boldsymbol{u}_{h} \cdot \boldsymbol{n}_{E}-\varphi_{h}\right)\left(\boldsymbol{x}_{c}\right)\right|=\frac{1}{2}\left|\llbracket \boldsymbol{u}_{h} \cdot \boldsymbol{n}_{E} \rrbracket\left(\boldsymbol{x}_{c}\right)\right| \quad \text { and } \quad\left(\boldsymbol{u}_{h} \cdot \boldsymbol{n}_{E}-\varphi_{h}\right)\left(\boldsymbol{x}_{i}\right)=0
$$

which yields (Simpson rule)

$$
\left\|\boldsymbol{u}_{h} \cdot \boldsymbol{n}_{E}-\varphi_{h}\right\|_{0, E}^{2}=\frac{1}{12} h_{E}\left|\llbracket \boldsymbol{u}_{h} \cdot \boldsymbol{n}_{E} \rrbracket\left(\boldsymbol{x}_{c}\right)\right|^{2}
$$


Thus, inserting 3.33 and 3.40 into 3.39 and choosing $\varepsilon:=\frac{1}{c_{4}+1}$ we obtain

$$
-\left\langle q_{h}^{\boldsymbol{u}_{h}}, \boldsymbol{u}_{h} \cdot \boldsymbol{n}\right\rangle \geqslant \frac{1}{2} \sum_{E \in \mathcal{G}_{h}} \frac{1}{h_{E}}\left\|\boldsymbol{u}_{h} \cdot \boldsymbol{n}_{E}\right\|_{0, E}^{2}-c_{5} \sum_{\boldsymbol{x} \in \mathcal{C}}\left|\llbracket \boldsymbol{u}_{h} \cdot \boldsymbol{n} \rrbracket(\boldsymbol{x})\right|^{2}
$$

with $c_{5}:=\frac{c_{4}+1}{12}$, which only depends on $\eta_{0}$.

(3) To prove (3.35), let us first introduce a continuous, element-wise linear function $q_{0}^{\boldsymbol{u}_{h}}$, which coincides with

$q_{h}^{\boldsymbol{u}_{h}}$ on the boundary $\Gamma$ and vanishes at all the interior nodes, and a continuous, element-wise linear function $\psi_{h}$ vanishing on the boundary and equal to 1 at all the interior nodes. Let us observe that, using an argument analogous to the one of Lemma 3.3 (omitted here), it holds

$$
\left\|q_{0}^{\boldsymbol{u}_{h}}\right\|_{0}^{2} \leqslant c_{\mathrm{NB}} \sum_{E \in \mathcal{G}_{h}} h_{E}\left\|q_{h}^{\boldsymbol{u}_{h}}\right\|_{0, E}^{2}
$$

where $c_{\mathrm{NB}}$ is the maximum number of triangles adjacent to a boundary node. Moreover, we have

$$
q_{h}^{\boldsymbol{u}_{h}}=q_{0}^{\boldsymbol{u}_{h}}+c_{q} \psi_{h} .
$$

From $\int_{\boldsymbol{\Omega}} q_{h}^{\boldsymbol{u}_{h}}=0$ we obtain

$$
c_{q}=-\frac{\int_{\Omega} q_{0}^{\boldsymbol{u}_{h}}}{\int_{\Omega} \psi_{h}} \quad \text { and } \quad\left|c_{q}\right| \leqslant \frac{\left\|q_{0}^{\boldsymbol{u}_{h}}\right\|_{0}\left|B_{h}\right|^{\frac{1}{2}}}{\int_{\Omega} \psi_{h}}
$$

since $q_{0}^{\boldsymbol{u}_{h}}$ is different from 0 only on $B_{h}$. Hence, using the assumption 2.5,

$$
\left\|q_{h}^{\boldsymbol{u}_{h}}\right\|_{0} \leqslant\left(1+\frac{\left\|\psi_{h}\right\|_{0}\left|B_{h}\right|^{\frac{1}{2}}}{\int_{\boldsymbol{\Omega}} \psi_{h}}\right)\left\|q_{0}^{\boldsymbol{u}_{h}}\right\|_{0} \leqslant\left(1+\frac{|\boldsymbol{\Omega}|^{\frac{1}{2}}\left|B_{h}\right|^{\frac{1}{2}}}{|\boldsymbol{\Omega}|-\left|B_{h}\right|}\right)\left\|q_{0}^{\boldsymbol{u}_{h}}\right\|_{0} \leqslant\left(1+\frac{\omega^{\frac{1}{2}}}{1-\omega}\right)\left\|q_{0}^{\boldsymbol{u}_{h}}\right\|_{0} .
$$

Since $0<\omega<1$, the coefficient inside the parentheses is always strictly larger than one (and it approaches one on fine meshes). Inserting (3.41) into (3.42) and using (3.33) we obtain 3.35 with a constant $c_{6}$ depending on $c_{\mathrm{NB}}, \eta_{0}$, and $\omega$. Finally, 3.36) can be obtained combining the inverse inequality (3.2) on each triangle and 3.35 :

$$
\sum_{\boldsymbol{T} \in \mathcal{T}_{h}} h_{\boldsymbol{T}}^{2}\left\|\nabla q_{h}^{\boldsymbol{u}_{h}}\right\|_{0, \boldsymbol{T}}^{2} \leqslant c_{I}^{2} \sum_{\boldsymbol{T} \in \mathcal{T}_{h}}\left\|q_{h}^{\boldsymbol{u}_{h}}\right\|_{0, \boldsymbol{T}}^{2} \leqslant c_{6} c_{I}^{2} \sum_{E \in \mathcal{G}_{h}} \frac{1}{h_{E}}\left\|\boldsymbol{u}_{h} \cdot \boldsymbol{n}_{E}\right\|_{0, E}^{2}
$$

Finally, we are able to show control of the normal velocity for arbitrary values of physical parameters.

Lemma 3.6 (Boundary control - II). Let $\delta, \rho>0, \alpha \geqslant 0$, and $\mu_{\mathrm{eff}}, \sigma \geqslant 0$ with $\mu_{\mathrm{eff}}+\sigma>0$ and let us assume that the family of triangulations $\left\{\mathcal{T}_{h}\right\}_{h}$ satisfies (3.31) and (2.5). Then, for any $\left(\boldsymbol{u}_{h}, p_{h}\right) \in \boldsymbol{V}_{h} \times Q_{h}$, there exists a function $q_{h} \in Q_{h}$ and a constant $C_{3}=C_{3}(\alpha, \delta, \rho)>0$ independent from the physical parameters, from $\boldsymbol{u}_{h}$, and from $h$, such that

$$
A_{h}\left[\left(\boldsymbol{u}_{h}, p_{h}\right) ;\left(\mathbf{0}, q_{h}\right)\right] \geqslant \frac{1}{4} \sum_{E \in \mathcal{G}_{h}} \frac{\nu}{h_{E}}\left\|\boldsymbol{u}_{h} \cdot \boldsymbol{n}_{E}\right\|_{0, E}^{2}-C_{3}\left(\left.\left\|\boldsymbol{u}_{h}\right\|\left|+\rho \nu \sum_{\boldsymbol{x} \in \mathcal{C}}\right| \llbracket \boldsymbol{u}_{h} \cdot \boldsymbol{n} \rrbracket(\boldsymbol{x})\right|^{2}+\alpha \sum_{\boldsymbol{T} \in \mathcal{T}_{h}} \frac{h_{\boldsymbol{T}}^{2}}{\nu}\left\|\nabla p_{h}\right\|_{0, \boldsymbol{T}}^{2}\right) .
$$


Proof: Let $\left(\boldsymbol{u}_{h}, p_{h}\right) \in \boldsymbol{V}_{h} \times Q_{h}$ and let $q_{h}:=\nu q_{h}^{\boldsymbol{u}_{h}}$, where $q_{h}^{\boldsymbol{u}_{h}}$ is the function defined in Lemma 3.5. Using the Cauchy-Schwarz inequality, Young inequality, (3.34), (3.35), and (3.36), we obtain

$$
\begin{aligned}
A_{h}\left[\left(\boldsymbol{u}_{h}, p_{h}\right) ;\left(\mathbf{0}, q_{h}\right)\right]= & \left(\nabla \cdot \boldsymbol{u}_{h}, \nu q_{h}^{\boldsymbol{u}_{h}}\right)+\alpha \sum_{\boldsymbol{T} \in \mathcal{T}_{h}} \frac{h_{\boldsymbol{T}}^{2}}{\nu}\left(\sigma \boldsymbol{u}_{h}+\nabla p_{h}, \nu \nabla q_{h}^{\boldsymbol{u}_{h}}\right)_{\boldsymbol{T}}-\left\langle\nu q_{h}^{\boldsymbol{u}_{h}}, \boldsymbol{u}_{h} \cdot \boldsymbol{n}\right\rangle \\
\geqslant & -\frac{1}{2 \varepsilon} \nu \delta\left\|\nabla \cdot \boldsymbol{u}_{h}\right\|_{0}^{2}-\frac{\varepsilon \nu}{2 \delta}\left\|q_{h}^{\boldsymbol{u}_{h}}\right\|_{0}^{2}-\alpha \sum_{\boldsymbol{T} \in \mathcal{T}_{h}} \frac{h_{\boldsymbol{T}}^{2}}{\nu}\left(\sigma^{2} \frac{1}{2 \varepsilon^{\prime}}\left\|\boldsymbol{u}_{h}\right\|_{0, \boldsymbol{T}}^{2}+\nu^{2} \frac{\varepsilon^{\prime}}{2}\left\|\nabla q_{h}^{\boldsymbol{u}_{h}}\right\|_{0, \boldsymbol{T}}^{2}\right) \\
& -\alpha \sum_{\boldsymbol{T} \in \mathcal{T}_{h}} \frac{h_{\boldsymbol{T}}^{2}}{\nu}\left(\frac{1}{2 \varepsilon^{\prime}}\left\|\nabla p_{h}\right\|_{0, \boldsymbol{T}}^{2}+\nu^{2} \frac{\varepsilon^{\prime}}{2}\left\|\nabla q_{h}^{\boldsymbol{u}_{h}}\right\|_{0, \boldsymbol{T}}^{2}\right)-\nu\left\langle q_{h}^{\boldsymbol{u}_{h}}, \boldsymbol{u}_{h} \cdot \boldsymbol{n}\right\rangle \\
\geqslant & \left(\frac{1}{2}-\frac{\varepsilon}{2 \delta} c_{6}-\alpha c_{I}^{2} c_{6} \varepsilon^{\prime}\right) \sum_{E \in \mathcal{G}_{h}} \frac{\nu}{h_{E}}\left\|\boldsymbol{u}_{h} \cdot \boldsymbol{n}_{E}\right\|_{0, E}^{2}-\frac{c_{5}}{\rho} \rho \nu \sum_{\boldsymbol{x} \in \mathcal{C}}\left|\llbracket \boldsymbol{u}_{h} \cdot \boldsymbol{n} \rrbracket(\boldsymbol{x})\right|^{2} \\
& -\frac{1}{2 \varepsilon} \nu \delta\left\|\nabla \cdot \boldsymbol{u}_{h}\right\|_{0}^{2}-\alpha \frac{1}{2 \varepsilon^{\prime}} \sum_{\boldsymbol{T} \in \mathcal{T}_{h}}^{\frac{\sigma h_{\boldsymbol{T}}^{2}}{\nu}} \underbrace{\nu}_{\leqslant 1}\left\|\boldsymbol{u}_{h}\right\|_{0, \boldsymbol{T}}^{2}-\alpha \frac{1}{2 \varepsilon^{\prime}} \sum_{\boldsymbol{T} \in \mathcal{T}_{h}} \frac{h_{\boldsymbol{T}}^{2}}{\nu}\left\|\nabla p_{h}\right\|_{0, \boldsymbol{T}}^{2},
\end{aligned}
$$

for any $\varepsilon, \varepsilon^{\prime}>0$. The proof is completed choosing $\varepsilon=\frac{\delta}{4 c_{6}}$ and $\varepsilon^{\prime}=\frac{1}{8 \alpha c_{6} c_{I}^{2}}$, and defining

$$
C_{3}:=\max \left\{\frac{c_{5}}{\rho}, \frac{2 c_{6}}{\delta}, 4 \alpha^{2} c_{6} c_{I}^{2}, 4 \alpha c_{6} c_{I}^{2}\right\}
$$

which depends only on the shape-regularity of the mesh and on the three stabilization parameters.

Remark 3.5. As stated in Section 2.3, the scaling of the stabilization terms by $\nu$ is a necessary requirement in order to obtain stability estimates independent from the physical parameters. In the argument used for the last proof, using an element-dependent scaling $\nu_{\boldsymbol{T}}:=\mu_{\mathrm{eff}}+\sigma h_{\boldsymbol{T}}^{2}$ (a suitable alternative for the case of essential boundary conditions, see, e.g., [3] ) instead of $\nu$ for the Grad-Div stabilization does not allow to uniformly bound the term $\left(\nabla \cdot \boldsymbol{u}_{h}, \nu q_{h}^{\boldsymbol{u}_{h}}\right)$.

Remark 3.6 (On the behavior of $C_{3}$ ). Notice that, in order to assure the validity of Lemma 3.6 both, Grad-Div and corner stabilization, are required (i.e., $\delta, \rho>0)$. In particular, it holds $C_{3}=O\left(\delta^{-1}+\rho^{-1}\right)$ for small values of $\delta$ and $\rho$. Moreover, as already observed in Remark 3.4 $\alpha>0$ is not strictly required.

The previously proven Lemmata allow to prove inf-sup stability of the considered formulation (2.7), which is stated in the following theorem.

Theorem 3.1 (Inf-sup stability).

Let $\alpha, \delta, \rho>0$ and $\mu_{\mathrm{eff}}, \sigma \geqslant 0$ with $\mu_{\mathrm{eff}}+\sigma>0$, and let us assume that the family of triangulations $\left\{\mathcal{T}_{h}\right\}_{h}$ fulfills the assumptions stated in Lemma 3.5. Then there exists a constant $\beta>0$, independent from the physical parameters and from $h$, such that

$$
\inf _{\left(\boldsymbol{u}_{h}, p_{h}\right) \in \boldsymbol{V}_{h} \times Q_{h} \backslash\{(\mathbf{0}, 0)\}}\left(\sup _{\left(\boldsymbol{v}_{h}, q_{h}\right) \in \boldsymbol{V}_{h} \times Q_{h} \backslash\{(\mathbf{0}, 0)\}}\left(\frac{A_{h}\left[\left(\boldsymbol{u}_{h}, p_{h}\right) ;\left(\boldsymbol{v}_{h}, q_{h}\right)\right]}{\left\|\left(\boldsymbol{u}_{h}, p_{h}\right)\right\|\left\|_{h}\right\|\left(\boldsymbol{v}_{h}, q_{h}\right) \|_{h}}\right)\right) \geqslant \beta .
$$

Moreover, $\beta^{-1}=O\left(\alpha^{-1}\left(\delta^{-1}+\rho^{-1}\right)\right)$, for $\alpha, \delta, \rho \ll 1$. 
Proof: Let $\left(\boldsymbol{u}_{h}, p_{h}\right) \in \boldsymbol{V}_{h} \times Q_{h}$. For the sake of simplicity, let us introduce the following notation:

$$
\begin{aligned}
& \xi_{0}:=\left\|\left(\boldsymbol{u}_{h}, p_{h}\right)\right\|_{h}^{2}-\frac{\left\|p_{h}\right\|_{0}^{2}}{\nu}-\sum_{E \in \mathcal{G}_{h}} \theta \frac{\mu_{\mathrm{eff}}}{h_{E}}\left\|\boldsymbol{u}_{h}\right\|_{0, E}^{2}-\sum_{E \in \mathcal{G}_{h}} \frac{\nu}{h_{E}}\left\|\boldsymbol{u}_{h} \cdot \boldsymbol{n}_{E}\right\|_{0, E}^{2}, \\
& \xi_{1}:=\frac{\left\|p_{h}\right\|_{0}^{2}}{\nu} \\
& \xi_{2}:=\sum_{E \in \mathcal{G}_{h}} \theta \frac{\mu_{\mathrm{eff}}}{h_{E}}\left\|\boldsymbol{u}_{h}\right\|_{0, E}^{2} \\
& \xi_{3}:=\sum_{E \in \mathcal{G}_{h}} \frac{\nu}{h_{E}}\left\|\boldsymbol{u}_{h} \cdot \boldsymbol{n}_{E}\right\|_{0, E}^{2}
\end{aligned}
$$

such that $\left\|\left(\boldsymbol{u}_{h}, p_{h}\right)\right\|_{h}^{2}=\xi_{0}+\xi_{1}+\xi_{2}+\xi_{3}$. Now we can rewrite and summarize the estimates proven in Lemmata $3.1,3.2,3.4$, and 3.6 as

$$
\begin{aligned}
& A_{h}\left[\left(\boldsymbol{u}_{h}, p_{h}\right) ; C_{0}^{-1}\left(\boldsymbol{u}_{h}, p_{h}\right)\right] \geqslant \xi_{0}, \\
& A_{h}\left[\left(\boldsymbol{u}_{h}, p_{h}\right) ; 2\left(\boldsymbol{v}_{h}, 0\right)\right] \geqslant \xi_{1}-2 C_{1}\left(\xi_{0}+\xi_{2}\right) \geqslant \xi_{1}-2 C_{1}\left(\xi_{0}+\xi_{2}+\xi_{3}\right), \\
& A_{h}\left[\left(\boldsymbol{u}_{h}, p_{h}\right) ; 4 c_{0}^{-1}\left(\theta \boldsymbol{w}_{h}^{\boldsymbol{u}_{h}}, 0\right)\right] \geqslant \xi_{2}-\frac{4 C_{2}}{c_{0}} \xi_{0}, \\
& A_{h}\left[\left(\boldsymbol{u}_{h}, p_{h}\right) ; 4\left(\mathbf{0}, \nu q_{h}^{\boldsymbol{u}_{h}}\right)\right] \geqslant \xi_{3}-4 C_{3} \xi_{0} .
\end{aligned}
$$

Summing up the last two inequalities leads to

$$
A_{h}\left[\left(\boldsymbol{u}_{h}, p_{h}\right) ;\left(4 c_{0}^{-1} \theta \boldsymbol{w}_{h}^{\boldsymbol{u}_{h}}, 4 \nu q_{h}^{\boldsymbol{u}_{h}}\right)\right] \geqslant\left(\xi_{2}+\xi_{3}\right)-\hat{C}_{2} \xi_{0},
$$

where $\widehat{C}_{2}:=\frac{4 C_{2}}{c_{0}}+4 C_{3}$. Consider first a test function $\left(\boldsymbol{z}_{h}^{1}, r_{h}^{1}\right) \in \boldsymbol{V}_{h} \times Q_{h}$ of the form

$$
\left(\boldsymbol{z}_{h}^{1}, r_{h}^{1}\right):=\left(1-\eta_{1}\right) C_{0}^{-1}\left(\boldsymbol{u}_{h}, p_{h}\right)+\eta_{1}\left(4 c_{0}^{-1} \theta \boldsymbol{w}_{h}^{\boldsymbol{u}_{h}}, 4 \nu q_{h}^{\boldsymbol{u}_{h}}\right),
$$

depending on a parameter $\eta_{1} \in(0,1)$ which will be determined later. It holds

$$
A_{h}\left[\left(\boldsymbol{u}_{h}, p_{h}\right) ;\left(\boldsymbol{z}_{h}^{1}, r_{h}^{1}\right)\right] \geqslant\left(1-\eta_{1}-\widehat{C}_{2} \eta_{1}\right) \xi_{0}+\eta_{1}\left(\xi_{2}+\xi_{3}\right) .
$$

Hence, defining

$$
\eta_{1}:=\frac{1}{\widehat{C}_{2}+2} \in\left(0, \frac{1}{2}\right)
$$

gives $\left(1-\eta_{1}-\widehat{C}_{2} \eta_{1}\right)=\eta_{1}$ and thus

$$
A_{h}\left[\left(\boldsymbol{u}_{h}, p_{h}\right) ;\left(\boldsymbol{z}_{h}^{1}, r_{h}^{1}\right)\right] \geqslant \frac{1}{\widehat{C}_{2}+2}\left(\xi_{0}+\xi_{2}+\xi_{3}\right) .
$$

Next, consider a test function $\left(\boldsymbol{z}_{h}^{2}, r_{h}^{2}\right) \in \boldsymbol{V}_{h} \times Q_{h}$ of the form

$$
\left(\boldsymbol{z}_{h}^{2}, r_{h}^{2}\right):=\left(1-\eta_{2}\right)\left(\widehat{C}_{2}+2\right)\left(\boldsymbol{z}_{h}^{1}, r_{h}^{1}\right)+\eta_{2}\left(2 \boldsymbol{v}_{h}, 0\right),
$$

depending on a parameter $\eta_{2} \in(0,1)$ to be determined later. This yields

$$
A_{h}\left[\left(\boldsymbol{u}_{h}, p_{h}\right) ;\left(\boldsymbol{z}_{h}^{2}, r_{h}^{2}\right)\right] \geqslant\left(1-\eta_{2}-2 C_{1} \eta_{2}\right)\left(\xi_{0}+\xi_{2}+\xi_{3}\right)+\eta_{2} \xi_{1}
$$


Therefore, the choice

leads to

$$
\eta_{2}:=\frac{1}{2 C_{1}+2} \in\left(0, \frac{1}{2}\right)
$$

$$
A_{h}\left[\left(\boldsymbol{u}_{h}, p_{h}\right) ;\left(\boldsymbol{z}_{h}^{2}, r_{h}^{2}\right)\right] \geqslant \frac{1}{2 C_{1}+2}\left(\xi_{0}+\xi_{1}+\xi_{2}+\xi_{3}\right)=\frac{1}{2 C_{1}+2}\left\|\left(\boldsymbol{u}_{h}, p_{h}\right)\right\|_{h}^{2} .
$$

It remains to control the norm of the above constructed test function $\left(\boldsymbol{z}_{h}^{2}, r_{h}^{2}\right)$. From the properties of $\boldsymbol{v}_{h}$ stated in 3.10 we have

$$
\left\|\left(\boldsymbol{v}_{h}, 0\right)\right\|_{h}^{2}=\mu_{\mathrm{eff}}\left\|\nabla \boldsymbol{v}_{h}\right\|_{0}^{2}+\sigma\left\|\boldsymbol{v}_{h}\right\|_{0}^{2}+\delta \nu\left\|\nabla \cdot \boldsymbol{v}_{h}\right\|_{0}^{2} \leqslant c_{\Omega}^{2}(1+n \delta) \frac{\left\|p_{h}\right\|_{0}^{2}}{\nu} .
$$

Moreover, from Lemmas 3.3, and Equations 3.35 and 3.36 we infer

$$
\begin{gathered}
\left\|\left(\theta \boldsymbol{w}_{h}^{\boldsymbol{u}_{h}}, 0\right)\right\|_{h}^{2} \leqslant\left(c_{3}^{2}+c_{2}+\delta n c_{2}+1\right)\left\|\left(\boldsymbol{u}_{h}, p_{h}\right)\right\|_{h}^{2}, \\
\left\|\left(\mathbf{0}, \nu q_{h}^{\boldsymbol{u}_{h}}\right)\right\|_{h}^{2}=\frac{1}{\nu}\left\|\nu q_{h}^{\boldsymbol{u}_{h}}\right\|_{0}^{2}+\alpha \sum_{\boldsymbol{T} \in \mathcal{T}_{h}} \frac{h_{\boldsymbol{T}}^{2}}{\nu}\left\|\nu \nabla q_{h}^{\boldsymbol{u}_{h}}\right\|_{0, \boldsymbol{T}}^{2} \leqslant c_{6}\left(1+\alpha c_{I}^{2}\right) \sum_{E \in \mathcal{G}_{h}} \frac{\nu}{h_{E}}\left\|\boldsymbol{u}_{h} \cdot \boldsymbol{n}_{E}\right\|_{0, E}^{2} .
\end{gathered}
$$

Hence, since $0<\eta_{1}<\frac{1}{2}$ and $0<\eta_{2}<\frac{1}{2}$, we can estimate

$$
\begin{aligned}
\left\|\left(\boldsymbol{z}_{h}^{2}, r_{h}^{2}\right)\right\|_{h}^{2} \leqslant & \eta_{2}^{2}\left\|\left(2 \boldsymbol{v}_{h}, 0\right)\right\|_{h}^{2}+\left(1-\eta_{2}\right)^{2}\left(\widehat{C}_{2}+2\right)^{2}\left\|\left(\boldsymbol{z}_{h}^{1}, r_{h}^{1}\right)\right\|_{h}^{2} \\
\leqslant & \left\|\left(\boldsymbol{v}_{h}, 0\right)\right\|_{h}^{2}+\left(1-\eta_{2}\right)^{2}\left(\widehat{C}_{2}+2\right)^{2} \eta_{1}^{2}\left(16 c_{0}^{-2}\left\|\left(\theta \boldsymbol{w}_{h}^{\boldsymbol{u}_{h}}, 0\right)\right\|_{h}^{2}+16\left\|\left(\mathbf{0}, \nu q_{h}^{\boldsymbol{u}_{h}}\right)\right\|_{h}^{2}\right) \\
& +\left(1-\eta_{1}\right)^{2} C_{0}^{-2}\left\|\left(\boldsymbol{u}_{h}, p_{h}\right)\right\|_{h}^{2} \\
& <\left(c_{\boldsymbol{\Omega}}^{2}(1+n \delta)+4\left(\widehat{C}_{2}+2\right)^{2}\left(\frac{\left(c_{3}^{2}+c_{2}+\delta n c_{2}+1\right)}{c_{0}^{2}}+c_{6}\left(1+\alpha c_{I}^{2}\right)\right)+C_{0}^{-2}\right)\left\|\left(\boldsymbol{u}_{h}, p_{h}\right)\right\|_{h}^{2}
\end{aligned}
$$

which allows to conclude

$$
A_{h}\left[\left(\boldsymbol{u}_{h}, p_{h}\right) ;\left(\boldsymbol{z}_{h}^{2}, r_{h}^{2}\right)\right] \geqslant \beta\left\|\left(\boldsymbol{u}_{h}, p_{h}\right)\right\|_{h}\|\|\left(\boldsymbol{z}_{h}^{2}, r_{h}^{2}\right) \|_{h}
$$

with

$$
\beta=\left(2 C_{1}+2\right)^{-1}\left(c_{\Omega}^{2}(1+n \delta)+4\left(\widehat{C}_{2}+2\right)^{2}\left(\frac{\left(c_{3}^{2}+c_{2}+\delta n c_{2}+1\right)}{c_{0}^{2}}+c_{6}\left(1+\alpha c_{I}^{2}\right)\right)+C_{0}^{-2}\right)^{-\frac{1}{2}}
$$

The behavior for small values of stabilization parameters follows from $C_{0}=O(1), C_{1}=O\left(\alpha^{-1}\right), C_{2}=O(1)$, and $C_{3}=O\left(\delta^{-1}+\rho^{-1}\right)$.

\subsection{Convergence}

Firstly, let us observe that the discrete, stabilized, penalty-free, non-symmetric Nitsche formulation 2.7 is consistent with problem (1.1):

Lemma 3.7 (Consistency and Galerkin Orthogonality).

Assume that $(\boldsymbol{u}, p) \in \boldsymbol{H}^{2}(\boldsymbol{\Omega}) \times H^{1}(\Omega)$ solves [1.1 satisfying either 1.2 (if $\left.\mu_{\mathrm{eff}}>0\right)$ or 1.3 (if $\mu_{\mathrm{eff}}=0$ ). Moreover, let $\left(\boldsymbol{u}_{h}, p_{h}\right) \in \boldsymbol{V}_{h} \times Q_{h}$ be the solution of 2.8. Then,

$$
A_{h}\left[\left(\boldsymbol{u}-\boldsymbol{u}_{h}, p-p_{h}\right) ;\left(\boldsymbol{v}_{h}, q_{h}\right)\right]=0, \quad \forall\left(\boldsymbol{v}_{h}, q_{h}\right) \in \boldsymbol{V}_{h} \times Q_{h} .
$$


The result follows from the consistency of the discrete formulation and the conformity of the triangulation.

The next lemma is related to the quality of the approximation with respect to the mesh-dependent norm.

Lemma 3.8 (Approximability).

Let $\sigma, \mu_{\mathrm{eff}} \geqslant 0$ with $\mu_{\mathrm{eff}}+\sigma>0$. Let $I_{h}^{L}(\cdot)$ and $M_{h}^{S Z}(\cdot)$ be the Lagrange interpolation operator onto $\boldsymbol{V}_{h}$ and the Scott-Zhang quasi-interpolation operator onto $Q_{h}$, respectively. Moreover, let us assume that $(\boldsymbol{u}, p) \in$ $\boldsymbol{H}^{2}(\boldsymbol{\Omega}) \times H^{1}(\boldsymbol{\Omega})$. Then it holds

$$
\left\|\left(\boldsymbol{u}-I_{h}^{L}(\boldsymbol{u}), p-M_{h}^{S Z}(p)\right)\right\|_{h}^{2} \leqslant c_{\mathrm{LSZ}}^{2} h^{2}\left[\nu\left(1+n \delta+2 c_{\mathrm{DT}}\right)\|\boldsymbol{u}\|_{2}^{2}+\frac{1}{\nu}(1+\alpha)\|p\|_{1}^{2}\right],
$$

where $c_{\mathrm{LSZ}}$ is a constant that depends only on 3.5 and $3.4 \mathrm{~b}$.

Proof: We start by estimating the bulk terms of the triple norm using the properties (3.5) of the interpolation operators:

$$
\begin{aligned}
& \mu_{\mathrm{eff}}\left\|\nabla\left(\boldsymbol{u}-I_{h}^{L}(\boldsymbol{u})\right)\right\|_{0}^{2}+\sigma\left\|\boldsymbol{u}-I_{h}^{L}(\boldsymbol{u})\right\|_{0}^{2}+\delta \nu\left\|\nabla \cdot\left(\boldsymbol{u}-I_{h}^{L}(\boldsymbol{u})\right)\right\|_{0}^{2} \\
&+\frac{1}{\nu}\left\|p-M_{h}^{S Z}(p)\right\|_{0}^{2}+\alpha \sum_{\boldsymbol{T} \in \mathcal{T}_{h}} \frac{h_{\boldsymbol{T}}^{2}}{\nu}\left\|\nabla\left(p-M_{h}^{S Z}(p)\right)\right\|_{0, \boldsymbol{T}}^{2} \\
& \leqslant \mu_{\mathrm{eff}} c_{\mathrm{La}}^{2} h^{2}\|\boldsymbol{u}\|_{2}^{2}+\sigma c_{\mathrm{La}}^{2} h^{4}\|\boldsymbol{u}\|_{2}^{2}+n \delta \nu c_{\mathrm{La}}^{2} h^{2}\|\boldsymbol{u}\|_{2}^{2} \\
&+\frac{1}{\nu} c_{\mathrm{SZ}}^{2} h^{2}\|p\|_{1}^{2}+\alpha \frac{h^{2}}{\nu} c_{\mathrm{SZ}}^{2}\|p\|_{1}^{2} \\
& \leqslant c_{\mathrm{LSZ}}^{2} h^{2}\left[(\underbrace{\mu_{\mathrm{eff}}+\sigma h^{2}}_{\leqslant \nu}+n \delta \nu)\|\boldsymbol{u}\|_{2}^{2}+\left(\frac{1}{\nu}(1+\alpha)\right)\|p\|_{1}^{2}\right] .
\end{aligned}
$$

For the additional boundary terms related to the penalty-free Nitsche method we get with (3.1):

$$
\begin{aligned}
\sum_{E \in \mathcal{G}_{h}} \theta \frac{\mu_{\mathrm{eff}}}{h_{E}} & \left\|\boldsymbol{u}-I_{h}^{L}(\boldsymbol{u})\right\|_{0, E}^{2}+\sum_{E \in \mathcal{G}_{h}} \frac{\nu}{h_{E}}\left\|\left(\boldsymbol{u}-I_{h}^{L}(\boldsymbol{u})\right) \cdot \boldsymbol{n}_{E}\right\|_{0, E}^{2} \\
& \leqslant c_{\mathrm{DT}} \sum_{\boldsymbol{T}_{E}: E \in \mathcal{G}_{h}}\left(\mu_{\mathrm{eff}}+\nu\right)\left(h_{\boldsymbol{T}_{E}}^{-2}\left\|\boldsymbol{u}-I_{h}^{L}(\boldsymbol{u})\right\|_{0, \boldsymbol{T}_{E}}^{2}+\left\|\nabla\left(\boldsymbol{u}-I_{h}^{L}(\boldsymbol{u})\right)\right\|_{0, \boldsymbol{T}_{E}}^{2}\right) \\
& \leqslant c_{\mathrm{DT}}\left(\mu_{\mathrm{eff}}+\nu\right) c_{\mathrm{La}}^{2} h^{2}\|\boldsymbol{u}\|_{2}^{2} .
\end{aligned}
$$

Finally, we observe that the interpolation error due to the corner stabilization term vanishes since the Lagrange interpolator is exact on mesh nodes. The inequality (3.43) is obtained summing up all the above estimated terms and observing that $\mu_{\mathrm{eff}}, \sigma h^{2} \leqslant \nu$.

Theorem 3.2 (A priori error estimate).

Let $\alpha, \delta, \rho>0$ and $\mu_{\mathrm{eff}}, \sigma \geqslant 0$ with $\mu_{\mathrm{eff}}+\sigma>0$. Let $(\boldsymbol{u}, p)$ be the solution of (1.1) and $\left(\boldsymbol{u}_{h}, p_{h}\right)$ be the solution of problem 2.7). Let us assume $(\boldsymbol{u}, p) \in \boldsymbol{H}^{2}(\boldsymbol{\Omega}) \times H^{1}(\boldsymbol{\Omega})$. Then it holds

$$
\left\|\left(\boldsymbol{u}-\boldsymbol{u}_{h}, p-p_{h}\right)\right\|_{h} \leqslant c_{\mathrm{LSZ}} h\left(C_{\boldsymbol{u}}\|\boldsymbol{u}\|_{2}+C_{p}\|p\|_{1}\right)
$$

with

$$
C_{\boldsymbol{u}}:=\nu^{\frac{1}{2}}\left[\left(1+n \delta+2 c_{\mathrm{DTI}}\right)^{\frac{1}{2}}+\frac{1}{\beta}\left(2\left(2 c_{\mathrm{DT}} c_{\mathrm{DTI}}\right)^{\frac{1}{2}}+n^{\frac{1}{2}}\left(\delta^{\frac{1}{2}}+1\right)+1+\left(2 c_{\mathrm{DT}}\right)^{\frac{1}{2}}+\alpha^{\frac{1}{2}}+\alpha\right)\right]
$$

and

$$
C_{p}:=\nu^{-\frac{1}{2}}\left[(1+\alpha)^{\frac{1}{2}}+\frac{1}{\beta}\left(\left(2 c_{\mathrm{DT}}\right)^{\frac{1}{2}}+\alpha^{\frac{1}{2}}+\alpha+\delta^{-\frac{1}{2}}\right)\right] .
$$


Proof: The proof is based on the combination of the inf-sup condition (Theorem 3.1), the Galerkin orthogonality (Lemma 3.7), Lemma 3.8 and the approximation properties of the (quasi-)interpolation operators. Let us consider the Lagrange interpolant $\boldsymbol{v}_{h}:=I_{h}^{L}(\boldsymbol{u})$ and the Scott-Zhang quasi-interpolant $q_{h}:=M_{h}^{S Z}(p)$, and decompose the error as

$$
\left\|\left(\boldsymbol{u}-\boldsymbol{u}_{h}, p-p_{h}\right)\right\|_{h} \leqslant\left\|\left(\boldsymbol{u}-\boldsymbol{v}_{h}, p-q_{h}\right)\right\|_{h}+\left\|\left(\boldsymbol{v}_{h}-\boldsymbol{u}_{h}, q_{h}-p_{h}\right)\right\|_{h} .
$$

Exploiting the inf-sup stability (Theorem 3.1) and the Galerkin orthogonality (Lemma 3.7), it holds:

$$
\left\|\left(\boldsymbol{u}-\boldsymbol{u}_{h}, p-p_{h}\right)\right\|_{h} \leqslant\left\|\left(\boldsymbol{u}-\boldsymbol{v}_{h}, p-q_{h}\right)\right\|_{h}+\frac{1}{\beta} \sup _{\left(\boldsymbol{w}_{h}, r_{h}\right) \in \boldsymbol{V}_{h} \times Q_{h} \backslash\{(\mathbf{0}, 0)\}} \frac{\left|A_{h}\left[\left(\boldsymbol{u}-\boldsymbol{v}_{h}, p-q_{h}\right) ;\left(\boldsymbol{w}_{h}, r_{h}\right)\right]\right|}{\left\|\left(\boldsymbol{w}_{h}, r_{h}\right) \mid\right\|_{h}} .
$$

Next, we bound $\left|A_{h}\left[\left(\boldsymbol{u}-\boldsymbol{v}_{h}, p-q_{h}\right) ;\left(\boldsymbol{w}_{h}, r_{h}\right)\right]\right|$. For the bulk terms related to the weak formulation of the Brinkman problem we obtain

$$
\begin{array}{r}
\left|\mu_{\mathrm{eff}}\left(\nabla\left(\boldsymbol{u}-\boldsymbol{v}_{h}\right), \nabla \boldsymbol{w}_{h}\right)\right| \leqslant \mu_{\mathrm{eff}}^{\frac{1}{2}}\left\|\nabla\left(\boldsymbol{u}-\boldsymbol{v}_{h}\right)\right\|_{0} \mu_{\mathrm{eff}}^{\frac{1}{2}}\left\|\nabla \boldsymbol{w}_{h}\right\|_{0} \leqslant c_{\mathrm{La}} \mu_{\mathrm{eff}}^{\frac{1}{2}} h\|\boldsymbol{u}\|_{2}\left\|\left(\boldsymbol{w}_{h}, r_{h}\right) \mid\right\|_{h}, \\
\left|\sigma\left(\boldsymbol{u}-\boldsymbol{v}_{h}, \boldsymbol{w}_{h}\right)\right| \leqslant \sigma^{\frac{1}{2}}\left\|\boldsymbol{u}-\boldsymbol{v}_{h}\right\|_{0} \sigma^{\frac{1}{2}}\left\|\boldsymbol{w}_{h}\right\|_{0} \leqslant c_{\mathrm{La}} \sigma^{\frac{1}{2}} h^{2}\|\boldsymbol{u}\|_{2}\left\|\left(\boldsymbol{w}_{h}, r_{h}\right) \mid\right\|_{h}, \\
\left|-\left(p-q_{h}, \nabla \cdot \boldsymbol{w}_{h}\right)\right| \leqslant(\delta \nu)^{-\frac{1}{2}}\left\|p-q_{h}\right\|_{0}(\delta \nu)^{\frac{1}{2}}\left\|\nabla \cdot \boldsymbol{w}_{h}\right\|_{0} \leqslant c_{\mathrm{SZ}}(\delta \nu)^{-\frac{1}{2}} h\|p\|_{1}\left\|\left(\boldsymbol{w}_{h}, r_{h}\right)\right\| \|_{h}, \\
\left|\left(\nabla \cdot\left(\boldsymbol{u}-\boldsymbol{v}_{h}\right), r_{h}\right)\right| \leqslant \nu^{\frac{1}{2}}\left\|\nabla \cdot\left(\boldsymbol{u}-\boldsymbol{v}_{h}\right)\right\|_{0} \nu^{-\frac{1}{2}}\left\|r_{h}\right\|_{0} \leqslant c_{\mathrm{La}}(\nu n)^{\frac{1}{2}} h\|\boldsymbol{u}\|_{2}\left\|\left(\boldsymbol{w}_{h}, r_{h}\right)\right\|_{h} .
\end{array}
$$

The GLS stabilization terms yield

$$
\begin{gathered}
\left|\alpha \sum_{\boldsymbol{T} \in \mathcal{T}_{h}} \frac{h_{\boldsymbol{T}}^{2}}{\nu}\left(\sigma\left(\boldsymbol{u}-\boldsymbol{v}_{h}\right), \sigma \boldsymbol{w}_{h}\right)_{\boldsymbol{T}}\right| \leqslant c_{\mathrm{La}} \alpha \sigma^{\frac{1}{2}} h^{2}\|\boldsymbol{u}\|_{2}\left\|\left(\boldsymbol{w}_{h}, r_{h}\right) \mid\right\|_{h}, \\
\left|\alpha \sum_{\boldsymbol{T} \in \mathcal{T}_{h}} \frac{h_{\boldsymbol{T}}^{2}}{\nu}\left(\sigma\left(\boldsymbol{u}-\boldsymbol{v}_{h}\right), \nabla r_{h}\right)_{\boldsymbol{T}}\right| \leqslant c_{\mathrm{La}}(\alpha \sigma)^{\frac{1}{2}} h^{2}\|\boldsymbol{u}\|_{2}\left\|\left(\boldsymbol{w}_{h}, r_{h}\right) \mid\right\|_{h}, \\
\left|\alpha \sum_{\boldsymbol{T} \in \mathcal{T}_{h}} \frac{h_{\boldsymbol{T}}^{2}}{\nu}\left(\nabla\left(p-q_{h}\right), \sigma \boldsymbol{w}_{h}\right)_{\boldsymbol{T}}\right| \leqslant c_{\mathrm{SZ}} \alpha \nu^{-\frac{1}{2}} h\|p\|_{1}\left\|\left(\boldsymbol{w}_{h}, r_{h}\right)\right\|_{h}, \\
\left|\alpha \sum_{\boldsymbol{T} \in \mathcal{T}_{h}} \frac{h_{\boldsymbol{T}}^{2}}{\nu}\left(\nabla\left(p-q_{h}\right), \nabla r_{h}\right)_{\boldsymbol{T}}\right| \leqslant c_{\mathrm{SZ}} \alpha^{\frac{1}{2}} \nu^{-\frac{1}{2}} h\|p\|_{1}\left\|\left(\boldsymbol{w}_{h}, r_{h}\right) \mid\right\|_{h},
\end{gathered}
$$

while for the terms related to the Grad-Div stabilization, we obtain

$$
\begin{aligned}
\left|\delta \sum_{\boldsymbol{T} \in \mathcal{T}_{h}} \nu\left(\nabla \cdot\left(\boldsymbol{u}-\boldsymbol{v}_{h}\right), \nabla \cdot \boldsymbol{w}_{h}\right)_{\boldsymbol{T}}\right| & \leqslant \sum_{\boldsymbol{T} \in \mathcal{T}_{h}}(\delta \nu)^{\frac{1}{2}}\left\|\nabla \cdot\left(\boldsymbol{u}-\boldsymbol{v}_{h}\right)\right\|_{0, \boldsymbol{T}}(\delta \nu)^{\frac{1}{2}}\left\|\nabla \cdot \boldsymbol{w}_{h}\right\|_{0, \boldsymbol{T}} \\
& \leqslant c_{\mathrm{La}}(n \delta \nu)^{\frac{1}{2}} h\|\boldsymbol{u}\|_{2}\left\|\left(\boldsymbol{w}_{h}, r_{h}\right)\right\| \|_{h} .
\end{aligned}
$$


The additional terms related to the penalty-free Nitsche method can be controlled as follows. First we treat:

$$
\begin{aligned}
\left|\left\langle\mu_{\mathrm{eff}} \nabla\left(\boldsymbol{u}-\boldsymbol{v}_{h}\right) \cdot \boldsymbol{n}, \boldsymbol{w}_{h}\right\rangle\right| \leqslant & \mu_{\mathrm{eff}}^{\frac{1}{2}}\left(\sum_{E \in \mathcal{G}_{h}} h_{E}\left\|\nabla\left(\boldsymbol{u}-\boldsymbol{v}_{h}\right) \cdot \boldsymbol{n}_{E}\right\|_{0, E}^{2}\right)^{\frac{1}{2}}\left(\sum_{E \in \mathcal{G}_{h}} \frac{\mu_{\mathrm{eff}}}{h_{E}}\left\|\boldsymbol{w}_{h}\right\|_{0, E}^{2}\right)^{\frac{1}{2}} \\
& \leqslant \frac{\mu_{\mathrm{eff}}^{\frac{1}{2}}}{\theta^{\frac{1}{2}}}\left(\sum_{\boldsymbol{T}_{E}: E \in \mathcal{G}_{h}} c_{\mathrm{DT}}\left(\left\|\nabla\left(\boldsymbol{u}-\boldsymbol{v}_{h}\right)\right\|_{0, \boldsymbol{T}_{E}}^{2}+h_{\boldsymbol{T}_{E}}^{2}\left\|\nabla\left(\nabla\left(\boldsymbol{u}-\boldsymbol{v}_{h}\right)\right)\right\|_{0, \boldsymbol{T}_{E}}^{2}\right)\right)^{\frac{1}{2}} \\
& \left\|\left(\boldsymbol{w}_{h}, r_{h}\right)\right\|_{h} \\
& \leqslant c_{\mathrm{La}}\left(2 \nu c_{\mathrm{DT}}\right)^{\frac{1}{2}} h\|\boldsymbol{u}\|_{2}\left\|\left(\boldsymbol{w}_{h}, r_{h}\right)\right\|_{h},
\end{aligned}
$$

and this implies

$$
\begin{aligned}
\left|\left\langle\mu_{\mathrm{eff}} \nabla \boldsymbol{w}_{h} \cdot \boldsymbol{n}, \boldsymbol{u}-\boldsymbol{v}_{h}\right\rangle\right| \leqslant & \mu_{\mathrm{eff}}^{\frac{1}{2}}\left(\sum_{E \in \mathcal{G}_{h}} \frac{1}{h_{E}}\left\|\boldsymbol{u}-\boldsymbol{v}_{h}\right\|_{0, E}^{2}\right)^{\frac{1}{2}}\left(\sum_{E \in \mathcal{G}_{h}} h_{E} \mu_{\mathrm{eff}}\left\|\nabla \boldsymbol{w}_{h} \cdot \boldsymbol{n}_{E}\right\|_{0, E}^{2}\right)^{\frac{1}{2}} \\
\leqslant & \left(\mu_{\mathrm{eff}} c_{\mathrm{DT}} c_{\mathrm{DTI}}\right)^{\frac{1}{2}}\left(\sum_{\boldsymbol{T}_{E}: E \in \mathcal{G}_{h}} h_{\boldsymbol{T}_{E}}^{-2}\left(\left\|\boldsymbol{u}-\boldsymbol{v}_{h}\right\|_{0, \boldsymbol{T}_{E}}^{2}+h_{\boldsymbol{T}_{E}}^{2}\left\|\nabla\left(\boldsymbol{u}-\boldsymbol{v}_{h}\right)\right\|_{0, \boldsymbol{T}_{E}}^{2}\right)\right)^{\frac{1}{2}} \\
& \times\left(\sum_{\boldsymbol{T}_{E}: E \in \mathcal{G}_{h}} \mu_{\mathrm{eff}}\left\|\nabla \boldsymbol{w}_{h}\right\|_{0, \boldsymbol{T}_{E}}^{2}\right)^{\frac{1}{2}} \\
\leqslant & c_{\mathrm{La}}\left(2 \mu_{\mathrm{eff}} c_{\mathrm{DT}} c_{\mathrm{DTI}}\right)^{\frac{1}{2}} h\|\boldsymbol{u}\|_{2}\left\|\left(\boldsymbol{w}_{h}, r_{h}\right)\right\|_{h} .
\end{aligned}
$$

Then we bound

$$
\begin{aligned}
& \left|\left\langle p-q_{h}, \boldsymbol{w}_{h} \cdot \boldsymbol{n}\right\rangle\right| \\
\leqslant & \left(\sum_{E \in \mathcal{G}_{h}} \frac{h_{E}}{\nu}\left\|p-q_{h}\right\|_{0, E}^{2}\right)^{\frac{1}{2}}\left(\sum_{E \in \mathcal{G}_{h}} \frac{\nu}{h_{E}}\left\|\boldsymbol{w}_{h} \cdot \boldsymbol{n}_{E}\right\|_{0, E}^{2}\right)^{\frac{1}{2}} \leqslant c_{\mathrm{SZ}}\left(2 c_{\mathrm{DT}}\right)^{\frac{1}{2}} \nu^{-\frac{1}{2}} h\|p\|_{1}\left\|\left(\boldsymbol{w}_{h}, r_{h}\right)\right\|_{h},
\end{aligned}
$$

and finally

$$
\begin{aligned}
& \left|\left\langle\left(\boldsymbol{u}-\boldsymbol{v}_{h}\right) \cdot \boldsymbol{n}, r_{h}\right\rangle\right| \\
\leqslant & \left(\sum_{E \in \mathcal{G}_{h}} \frac{1}{h_{E}}\left\|\boldsymbol{u}-\boldsymbol{v}_{h}\right\|_{0, E}^{2}\right)^{\frac{1}{2}}\left(\sum_{E \in \mathcal{G}_{h}} h_{E}\left\|r_{h}\right\|_{0, E}^{2}\right)^{\frac{1}{2}} \\
\leqslant & \left(c_{\mathrm{DT}} c_{\mathrm{DTI}} \nu\right)^{\frac{1}{2}}\left(\sum_{\boldsymbol{T}_{E}: E \in \mathcal{G}_{h}}\left(h_{\boldsymbol{T}_{E}}^{-2}\left\|\boldsymbol{u}-\boldsymbol{v}_{h}\right\|_{0, \boldsymbol{T}_{E}}^{2}+\left\|\nabla\left(\boldsymbol{u}-\boldsymbol{v}_{h}\right)\right\|_{0, \boldsymbol{T}_{E}}^{2}\right)\right)^{\frac{1}{2}}\left(\sum_{\boldsymbol{T}_{E}: E \in \mathcal{G}_{h}} \frac{\left\|r_{h}\right\|_{0, \boldsymbol{T}_{E}}^{2}}{\nu}\right)^{\frac{1}{2}} \\
\leqslant & c_{\mathrm{La}}\left(2 c_{\mathrm{DT}} c_{\mathrm{DTI}} \nu\right)^{\frac{1}{2}} h\|\boldsymbol{u}\|_{2}\left\|\left(\boldsymbol{w}_{h}, r_{h}\right)\right\|_{h} .
\end{aligned}
$$

The terms related to the corner stabilization vanish as the Lagrange interpolator is exact on mesh nodes. The proof is concluded summing up all the contributions and using Lemma 3.8 . 


\section{Numerical Examples}

The goal of this Section is to validate the results of the analysis of the penalty-free Nitsche method (2.7) against numerical experiments, especially testing the robustness of the formulation with respect to the physical parameters $\mu_{\text {eff }}$ and $\sigma$.

To this aim, we consider two examples defined on the unit square, i.e., $\boldsymbol{\Omega}:=(0,1)^{2}$ and discretized using uniform triangular meshes obtained by regular refinements (see Figure 1). In what follows, the four boundary components of $\boldsymbol{\Omega}$ will be referred to as $\Gamma_{i}, i=0,1,2,3$, with $\Gamma_{0}:=\{(x, 0): x \in[0,1]\}, \Gamma_{1}:=\{(1, y): y \in[0,1]\}$, $\Gamma_{2}:=\{(x, 1): x \in[0,1]\}, \Gamma_{3}:=\{(0, y): y \in[0,1]\}$.

\begin{tabular}{|c|c|c|c|}
\hline level & $h_{\boldsymbol{T}}$ & \# Cells (Triangles) & \# Dofs $(\boldsymbol{v}, p)$ \\
\hline \hline 0 & 1.41421 & 2 & $(8,4)$ \\
\hline 1 & 0.707107 & 8 & $(18,9)$ \\
\hline 2 & 0.353553 & 32 & $(50,25)$ \\
\hline 3 & 0.176777 & 128 & $(162,81)$ \\
\hline 4 & 0.0883883 & 512 & $(578,289)$ \\
\hline 5 & 0.0441942 & 2048 & $(2178,1089)$ \\
\hline 6 & 0.0220971 & 8192 & $(8450,4225)$ \\
\hline 7 & 0.0110485 & 32768 & $(33282,16641)$ \\
\hline 8 & 0.00552427 & 131072 & $(132098,66049)$ \\
\hline 9 & 0.00276214 & 524288 & $(526338,263169)$ \\
\hline
\end{tabular}

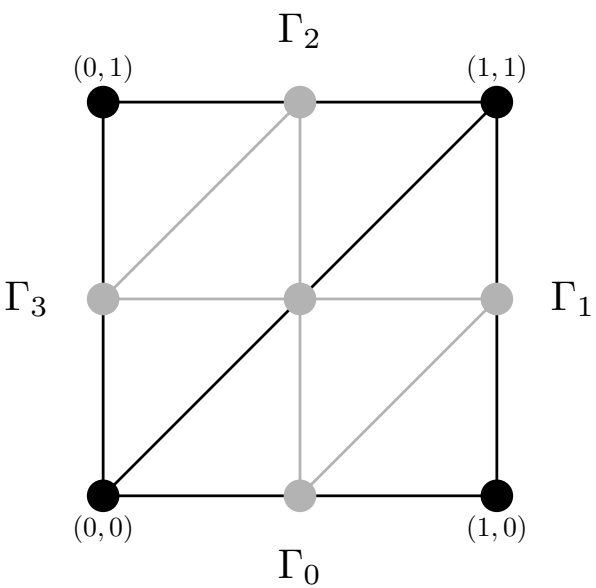

Figure 1. Left: Characteristic element size, number of elements, amount of degrees of freedom for the uniform triangular meshes used for the numerical computations. Right: Meshes corresponding to level 0 (black) and level 1 (black/grey).

In both examples, we compare the results considering different values of the stabilization parameters. The common legend for all forthcoming plots is shown in Figure 2. In particular, line colors will denote different values of $\alpha$ (GLS stabilization), line marker will refer to $\delta$ (Grad-Div stabilization) and line style will be related to the value of the characteristic length $L_{0}$. The numerical solutions have been computed using the finite element library ParMooN [33].

$$
\begin{aligned}
& -\varkappa-\alpha=0.1, \delta=0.1, \quad L_{0}=0.1 \quad-\varkappa-\alpha=1, \quad \delta=0.1, \quad L_{0}=0.1 \quad-x-\alpha=10, \delta=0.1, \quad L_{0}=0.1 \\
& -\Theta-\alpha=0.1, \delta=0.1, L_{0}=1 \quad-\Theta-\alpha=1, \delta=0.1, L_{0}=1 \quad-\Theta-\alpha=10, \delta=0.1, L_{0}=1 \\
& * \alpha=0.1, \delta=1, \quad L_{0}=0.1 \quad * \alpha=1, \quad \delta=1, \quad L_{0}=0.1 \quad * \alpha=10, \delta=1, \quad L_{0}=0.1 \\
& \multimap \alpha=0.1, \delta=1, \quad L_{0}=1 \quad \neg \alpha=1, \quad \delta=1, \quad L_{0}=1 \quad \text { - } \alpha=10, \delta=1, \quad L_{0}=1 \\
& \cdots \times \cdot \alpha=0.1, \delta=10, \quad L_{0}=0.1 \quad \cdots \times \cdot \alpha=1, \quad \delta=10, \quad L_{0}=0.1 \quad \cdots \times \cdot \alpha=10, \delta=10, \quad L_{0}=0.1
\end{aligned}
$$

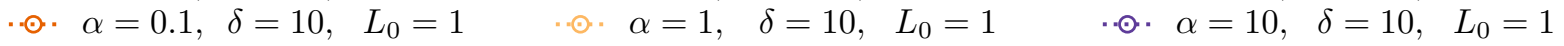

Figure 2. In each plot, we compare the errors varying the GLS stabilization parameter $\alpha$ (orange: 0.1, yellow: 1, purple: 10), the Grad-Div stabilization parameter $\delta$ (dashed line: 0.1, solid line: 1, dotted line: 10) and the characteristic length $L_{0}$. 


\subsection{Example I: A generalized Poiseuille/Brinkman flow}

The first example, taken from [21, is based on the functions

$$
\begin{aligned}
\boldsymbol{u}(x, y) & :=\left(u_{1}(x, y), 0\right)^{T}, \\
u_{1}(x, y) & :=\left\{\begin{array}{cc}
\sigma^{-1}\left(1-\frac{\exp \left((1-y) \sqrt{\frac{\sigma}{\mu_{\text {eff }}}}\right)+\exp \left(y \sqrt{\frac{\sigma}{\mu_{\text {eff }}}}\right)}{1+\exp \left(\sqrt{\frac{\sigma}{\mu_{\text {eff }}}}\right)}\right), & \text { if } \frac{\mu_{\text {eff }}}{\sigma}>0, \\
\sigma^{-1}, & \text { if } \frac{\mu_{\text {eff }}}{\sigma}=0,
\end{array}\right. \\
p(x, y): & =0.5-x,
\end{aligned}
$$

which solve the Brinkman problem (1.1) (for $\sigma>0$ and $\mu_{\text {eff }} \geqslant 0$ ) for $\boldsymbol{f}=\mathbf{0}$ and $g=0$, and with Neumann boundary conditions on the left and right boundaries

$$
(-\nabla \boldsymbol{u}+p \mathbb{I}) \cdot \boldsymbol{n}= \begin{cases}-0.5 \boldsymbol{n}, & \text { on } \Gamma_{1}, \\ +0.5 \boldsymbol{n}, & \text { on } \Gamma_{3},\end{cases}
$$

and homogeneous Dirichlet boundary conditions on top and bottom boundaries

$$
\boldsymbol{u}(x, y)=\mathbf{0}, \quad \text { on } \Gamma_{0} \cup \Gamma_{2} .
$$

Figure 3 depicts the velocity profile $\left(u_{1}(y)\right)$ for a few values of $\mu_{\text {eff }}$ and $\sigma$. Notice that, for smaller values of the ratio $\frac{\mu_{\text {eff }}}{\sigma}$, the solution has a boundary layer near the Dirichlet boundaries. As observed in [21, imposing strongly the Dirichlet boundary conditions might lead to strong unphysical oscillations (so-called overshoots and undershoots) near the boundary, since the mesh is not fine enough to resolve the boundary layer.
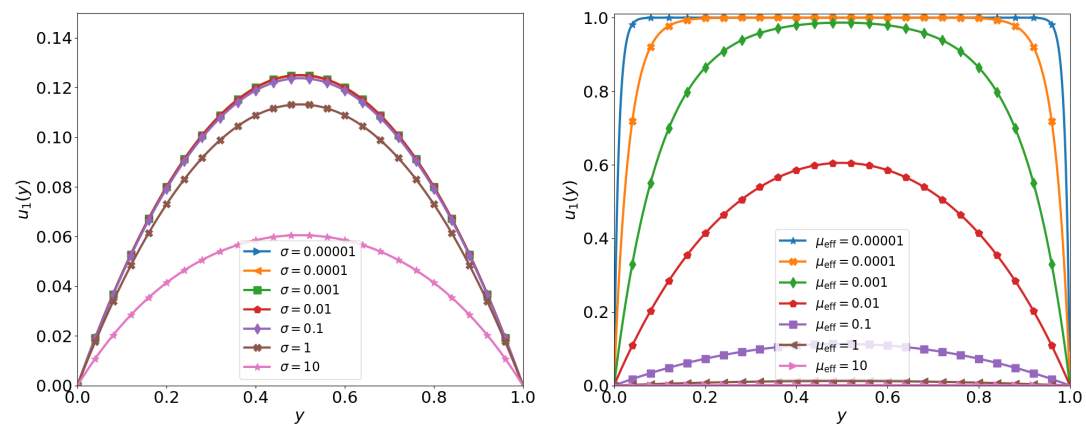

FiguRE 3. Example I: The function $u_{1}(y)$ defined in $4.1 \mathrm{~b}$ with fixed $\mu_{\mathrm{eff}}=1$ for different values of $\sigma$ (left) and with fixed $\sigma=1$ for different values of $\mu_{\text {eff }}$ (right).

We performed numerical simulations in two different physical regimes, considering $\mu_{\text {eff }}=\sigma=1$, i.e., $\frac{\mu_{\text {eff }}}{\sigma}=1$ and $\mu_{\text {eff }}=0.001, \sigma=10$, thus $\frac{\mu_{\text {eff }}}{\sigma}=0.0001$ ).

The convergence of the error in the mesh dependent norm 2.12 is shown in Figure 4 comparing the results for different values of the parameters $\alpha, \delta$, and $L_{0}$, described in Figure 2 We observe that, in both cases, the predicted convergence rate is obtained in the considered range of stabilization parameters. We also notice that the magnitude of the error slightly increases, the larger $\alpha$ is chosen. The Grad-Div stabilization parameter seems to have a similar effect on the results. Notice that, due to the absence of corners between Dirichlet boundaries, the corner stabilization is not necessary for this example. 

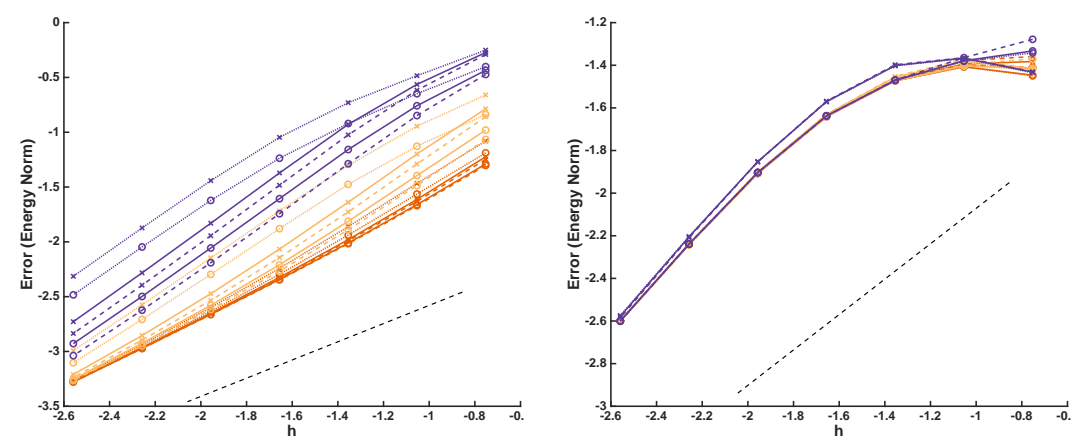

Figure 4. Example I: Error in the mesh dependent norm (2.12) against the mesh size (in double logarithmic scale), for the cases $\left(\mu_{\mathrm{eff}}, \sigma\right)=(1,1)$ (left) and $\left(\mu_{\mathrm{eff}}, \sigma\right)=(0.001,10)$ (right). A dashed line with slope equal to one is also shown.

In Figures 5 and 6 the different components of the velocity and pressure errors (including the error with respect to the velocity on the boundary) are depicted for the same values of the stabilization parameters, see Figure 2, The theoretical convergence order 1 is obtained in all cases.

For $\left(\mu_{\mathrm{eff}}, \sigma\right)=(1,1)$, the best results are given by the choice $\alpha=0.1, \delta=0.1$, and $L_{0}=1$, except for the divergence error of the velocity which reduces with increasing Grad-Div parameter (hence $\alpha=0.1, \delta=10$, and $\left.L_{0}=1\right)$.

The plots for $\left(\mu_{\mathrm{eff}}, \sigma\right)=(0.001,10)$ reveal that only the divergence, the normal velocity at the boundary and the pressure errors do depend significantly on the considered parameter variations. Moreover, except for the $L^{2}$-norm of the pressure error, the results with $L_{0}=1$ are in general better than the ones with $L_{0}=0.1$, when keeping the other parameters constant. In the excepted case, the situation is vice versa. It seems like the divergence error and the pressure error behavior contrary to each other with respect to the stabilization parameters.

\subsection{Example II: A trigonometric Darcy flow}

The next example ( 3,12$]$ ), focuses on a pure Darcy flow (i.e., $\mu_{\mathrm{eff}}=0$ ). Namely, we consider the velocitypressure pair

$$
\begin{aligned}
\boldsymbol{u}(x, y) & :=\left(\begin{array}{l}
u_{1}(x, y) \\
u_{2}(x, y)
\end{array}\right)=\left(\begin{array}{l}
-2 \pi \cos (2 \pi x) \sin (2 \pi y) \\
-2 \pi \sin (2 \pi x) \cos (2 \pi y)
\end{array}\right), \\
p(x, y) & :=\sigma \sin (2 \pi x) \sin (2 \pi y),
\end{aligned}
$$

which solves problem 1.1 for $\mu_{\mathrm{eff}}=0, \boldsymbol{f}=\mathbf{0}, g=8 \pi^{2} \sin (2 \pi x) \sin (2 \pi y)$, and with boundary conditions

$$
\boldsymbol{u} \cdot \boldsymbol{n}(x, y)=\left\{\begin{aligned}
2 \pi \sin (2 \pi x) & \text { on } \Gamma_{0}, \\
-2 \pi \sin (2 \pi y) & \text { on } \Gamma_{1}, \\
-2 \pi \sin (2 \pi x) & \text { on } \Gamma_{2}, \\
2 \pi \sin (2 \pi y) & \text { on } \Gamma_{3}
\end{aligned}\right.
$$

Numerical simulations have been performed considering $\sigma \in\{0.00001,0.001,0.01,0.1,1,10,1000,100000\}$. The errors in the mesh dependent norm (2.12), for $\sigma \in\{0.001,1,1000\}$ and different values of the stabilization parameters, are shown in Figure 7 . We recall that the legend is described in Figure 2 As apparent, the magnitude of the errors increases with increasing $\sigma$, whereas the overall behavior changes very slightly. Small GLS parameters and $L_{0}=0.1$ yield smaller energy errors than the other options. 

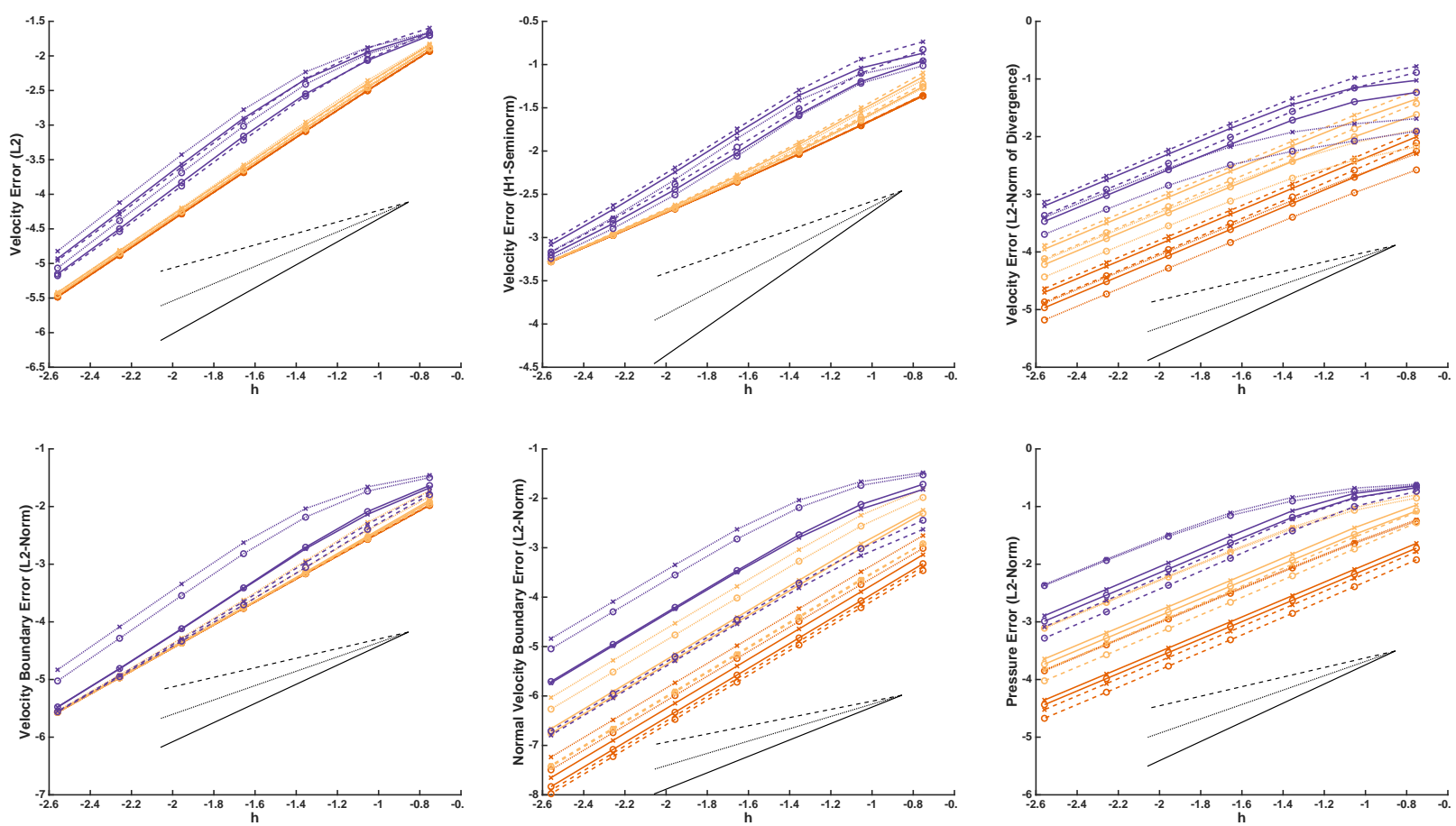

Figure 5. Example I: Velocity and pressure errors against the mesh size (in double logarithmic scale), for the case $\left(\mu_{\text {eff }}, \sigma\right)=(1,1)$, The lines with slope equal to 1 (dashed), $\frac{3}{2}$ (dotted) and 2 (solid) are also shown.

Figure 8 contains the error components with respect to the velocity for $\sigma=1$. This represents any value of $\sigma$, since this parameter has no influence on the behavior of the considered norms.

Finally, Figure 9 visualizes the reduction of the pressure error component with respect to different mesh resolutions for $\sigma \in\{0.001,1,1000\}$. As reflected in the energy norm, basically only the magnitude is influenced by a variation of $\sigma$ in a significant fashion.

Altogether, the predicted convergence rate was obtained for all combinations, nevertheless, the choice $\alpha=0.1$, $\delta=0.1$, and $L_{0}=0.1$ seems to lead to the best result. Note, that a variation in the corner stabilization parameter did not result in any change of the convergence behavior.

\section{Conclusions}

In this paper, we proposed and analyzed a stabilized equal-order finite element formulation for the Brinkman model combined with a non-symmetric Nitsche method. We investigated the properties of the recently introduced penalty-free Nitsche approach, which is used to weakly impose essential boundary conditions without the need of a penalty parameter.

We proved that the proposed penalty-free method is unconditionally stable with respect to the stabilization parameters - used for stabilizing equal-order finite element spaces and Grad-Div stabilization - and with respect to the physical parameters. In fact, we obtained inf-sup stability independently from the value of fluid viscosity and medium permeability, valid also in the limit regimes of Stokes $(\sigma=0)$ and Darcy $\left(\mu_{\text {eff }}=0\right)$ flows. Furthermore, optimal a priori error estimates in a mesh-dependent norm were derived, showing that the penaltyfree Nitsche formulation maintains the accuracy properties (in terms of convergence order and dependence on 

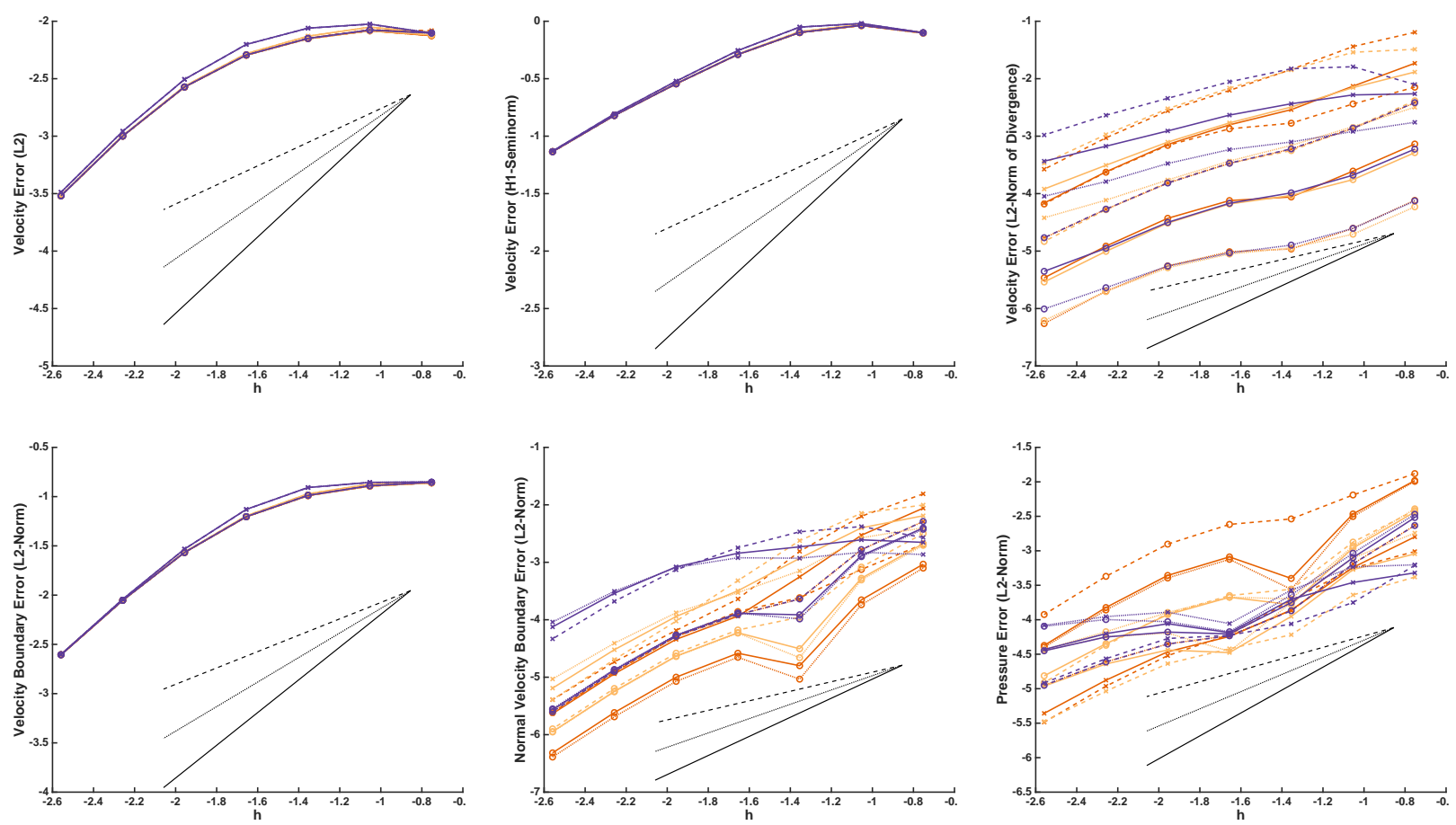

Figure 6. Example I: Velocity and pressure errors against the mesh size (in double logarithmic scale), for the case $\left(\mu_{\mathrm{eff}}, \sigma\right)=(0.001,10)$. The lines with slope equal to 1 (dashed), $\frac{3}{2}$ (dotted) and 2 (solid) are also shown.
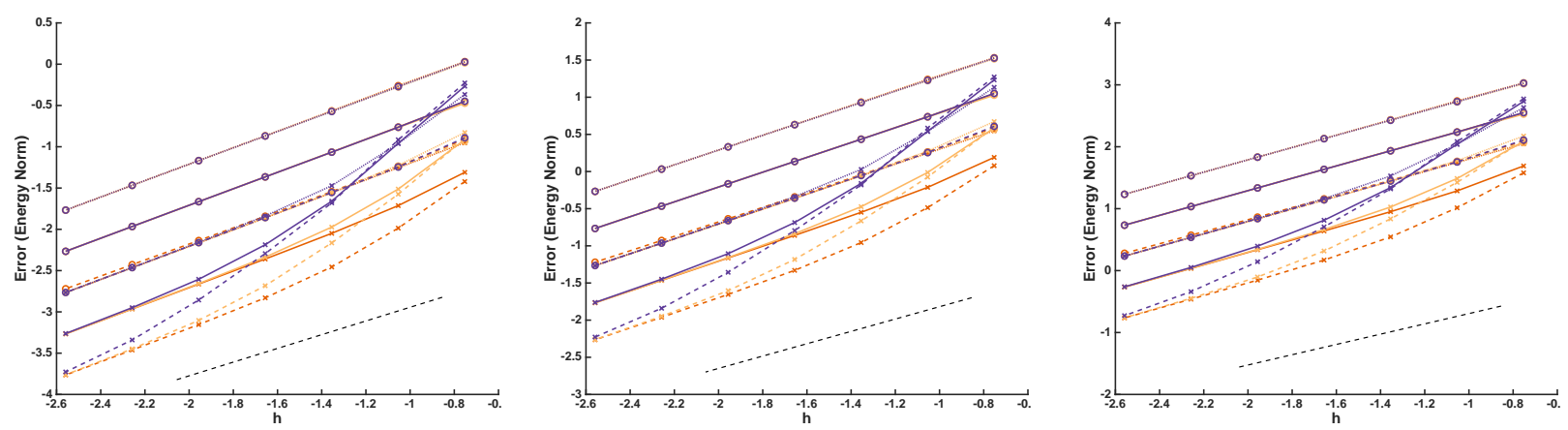

FIGURE 7. Example II: Error in the mesh dependent norm (2.12) against the mesh size (in double logarithmic scale), for the cases $\sigma=0.001$ (left), $\sigma=1$ (center), and $\sigma=1000$ (right). The dashed line visualizes a slope equal to 1 .

the physical parameters) of traditional approaches (strong imposition of essential boundary conditions) and of the symmetric Nitsche method.

Focusing on the case of two-dimensional polygonal boundaries, in order to prove the robust stability estimate we included an additional corner-stabilization, assuring stability of the normal velocity along the boundary in 

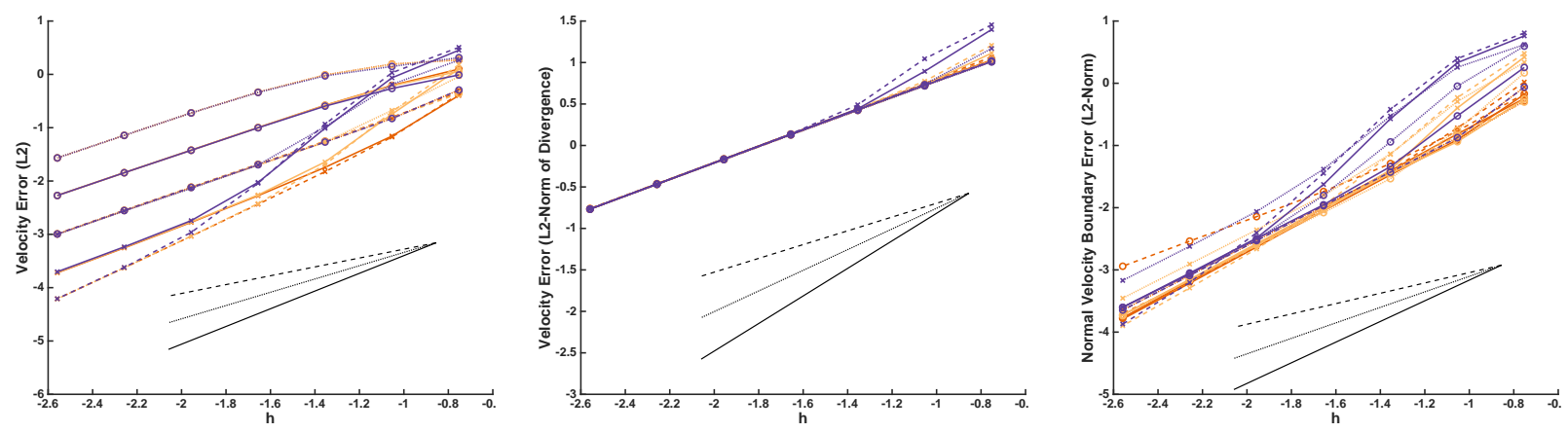

Figure 8. Example II: Velocity errors against the mesh size (in double logarithmic scale), for the case $\sigma=1$ as a representative case for any considered $\sigma$. The lines with slope equal to 1 (dashed), $\frac{3}{2}$ (dotted) and 2 (solid) are also shown.
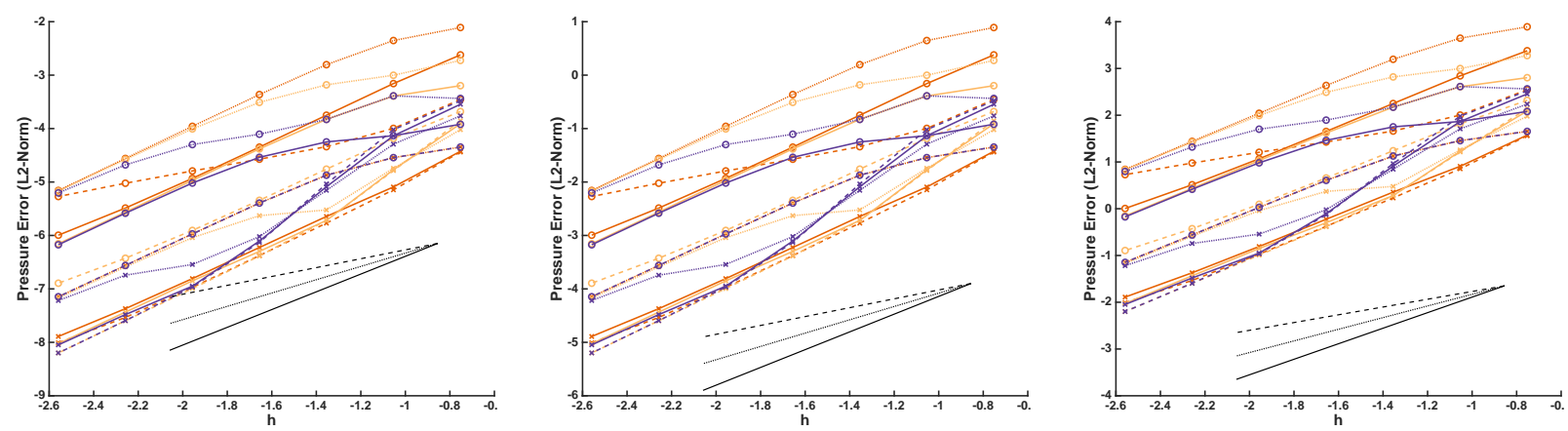

Figure 9. Example II: Pressure errors against the mesh size (in double logarithmic scale), for the cases $\sigma=0.001$ (left), $\sigma=1$ (center), and $\sigma=1000$ (right). The lines with slope equal to 1 (dashed), $\frac{3}{2}$ (dotted) and 2 (solid) are also shown.

the Darcy limit. The possibility of avoiding the corner stabilization, also in connection with different finite element pairs and in the context of three-dimensional applications, is a subject of current investigation.

\section{ACKNOWLEDGEMENTS}

F.C. thanks Thomas Boiveau and Erik Burman for fruitful discussions about Nitsche's method and the Brinkman problem. He thanks also Région Bourgogne Franche-Comté for partial funding ("Convention Région 2015C-4991. Modèles mathématiques et méthodes numériques pour l'élasticité non-linéaire").

\section{REFERENCES}

[1] Robert A. Adams. Sobolev spaces. Academic Press [A subsidiary of Harcourt Brace Jovanovich, Publishers], New York-London, 1975. Pure and Applied Mathematics, Vol. 65.

[2] Ivo Babuška. The finite element method with Lagrangian multipliers. Numer. Math., 20:179-192, 1972/73.

[3] Santiago Badia and Ramon Codina. Unified stabilized finite element formulations for the Stokes and the Darcy problems. SIAM J. Numer. Anal., 47(3):1971-2000, 2009.

[4] Thomas Boiveau. Penalty-free Nitsche method for interface problems in computational mechanics. PhD thesis, University College London, 2016. 
[5] Thomas Boiveau and Erik Burman. A penalty-free Nitsche method for the weak imposition of boundary conditions in compressible and incompressible elasticity. IMA J. Numer. Anal., 36(2):770-795, 2016.

[6] Susanne C. Brenner and L. Ridgway Scott. The mathematical theory of finite element methods, volume 15 of Texts in Applied Mathematics. Springer, New York, third edition, 2008.

[7] F. Brezzi. On the existence, uniqueness and approximation of saddle-point problems arising from Lagrangian multipliers. Rev. Française Automat. Informat. Recherche Opérationnelle Sér. Rouge, 8(R-2):129-151, 1974.

[8] F. Brezzi and J. Pitkäranta. On the stabilization of finite element approximations of the Stokes equations. In Efficient solutions of elliptic systems (Kiel, 1984), volume 10 of Notes Numer. Fluid Mech., pages 11-19. Friedr. Vieweg, Braunschweig, 1984.

[9] Franco Brezzi and Michel Fortin. Mixed and hybrid finite element methods, volume 15 of Springer Series in Computational Mathematics. Springer-Verlag, New York, 1991.

[10] H.C. Brinkman. A calculation of the viscous force exerted by a flowing fluid on a dense swarm of particles. Flow, Turbulence and Combustion, 1(1):27, 1949.

[11] Erik Burman. A penalty-free nonsymmetric Nitsche-type method for the weak imposition of boundary conditions. SIAM J. Numer. Anal., 50(4):1959-1981, 2012.

[12] Erik Burman and Peter Hansbo. A unified stabilized method for Stokes' and Darcy's equations. J. Comput. Appl. Math., 198(1):35-51, 2007.

[13] Philippe G. Ciarlet. The finite element method for elliptic problems, volume 40 of Classics in Applied Mathematics. Society for Industrial and Applied Mathematics (SIAM), Philadelphia, PA, 2002. Reprint of the 1978 original [North-Holland, Amsterdam; MR0520174 (58 \#25001)].

[14] $\mathrm{Ph}$. Clément. Approximation by finite element functions using local regularization. Rev. Française Automat. Informat. Recherche Opérationnelle Sér., 9(R-2):77-84, 1975.

[15] Carlo D'Angelo and Paolo Zunino. Robust numerical approximation of coupled Stokes' and Darcy's flows applied to vascular hemodynamics and biochemical transport. ESAIM Math. Model. Numer. Anal., 45(3):447-476, 2011.

[16] Jim Douglas, Jr. and Jun Ping Wang. An absolutely stabilized finite element method for the Stokes problem. Math. Comp., 52(186):495-508, 1989.

[17] Alexandre Ern and Jean-Luc Guermond. Theory and practice of finite elements, volume 159 of Applied Mathematical Sciences. Springer-Verlag, New York, 2004.

[18] Leopoldo P. Franca and Thomas J. R. Hughes. Two classes of mixed finite element methods. Comput. Methods Appl. Mech. Engrg., 69(1):89-129, 1988.

[19] J. Freund and R. Stenberg. On weakly imposed boundary conditions for second order problems. Proceedings of the international Conference on Finite Elements in Fluids - New trends and applications, Venezia, 1995.

[20] Vivette Girault and Pierre-Arnaud Raviart. Finite element methods for Navier-Stokes equations, volume 5 of Springer Series in Computational Mathematics. Springer-Verlag, Berlin, 1986. Theory and algorithms.

[21] A. Hannukainen, M. Juntunen, and R. Stenberg. Computations with finite element methods for the Brinkman problem. Computational Geosciences, 15(1):155-166, 2011.

[22] P. Hansbo and M. Juntunen. Weakly imposed Dirichlet boundary conditions for the Brinkman model of porous media flow. Appl. Numer. Math., 59(6):1274-1289, 2009.

[23] Thomas J. R. Hughes and Leopoldo P. Franca. A new finite element formulation for computational fluid dynamics. VII. The Stokes problem with various well-posed boundary conditions: symmetric formulations that converge for all velocity/pressure spaces. Comput. Methods Appl. Mech. Engrg., 65(1):85-96, 1987.

[24] Eleanor W. Jenkins, Volker John, Alexander Linke, and Leo G. Rebholz. On the parameter choice in grad-div stabilization for the stokes equations. Advances in Computational Mathematics, 40(2):491-516, Apr 2014.

[25] Mika Juntunen and Rolf Stenberg. Nitsche's method for general boundary conditions. Math. Comp., 78(267):1353-1374, 2009.

[26] Mika Juntunen and Rolf Stenberg. Analysis of finite element methods for the Brinkman problem. Calcolo, 47(3):129-147, 2010.

[27] K.A. Mardal, X.-C. Tai, and R. Winther. A robust finite element method for Darcy-Stokes flow. SIAM J. Numer. Anal., 40(5):1605-1631, 2002.

[28] Arif Masud and Thomas J. R. Hughes. A stabilized mixed finite element method for Darcy flow. Comput. Methods Appl. Mech. Engrg., 191(39-40):4341-4370, 2002.

[29] J. Nitsche. Über ein Variationsprinzip zur Lösung von Dirichlet-Problemen bei Verwendung von Teilräumen, die keinen Randbedingungen unterworfen sind. Abh. Math. Sem. Univ. Hamburg, 36:9-15, 1971. Collection of articles dedicated to Lothar Collatz on his sixtieth birthday.

[30] L. Ridgway Scott and Shangyou Zhang. Finite element interpolation of nonsmooth functions satisfying boundary conditions. Math. Comp., 54(190):483-493, 1990.

[31] Rolf Stenberg. On some techniques for approximating boundary conditions in the finite element method. J. Comput. Appl. Math., 63(1-3):139-148, 1995. International Symposium on Mathematical Modelling and Computational Methods Modelling 94 (Prague, 1994).

[32] Vidar Thomée. Galerkin finite element methods for parabolic problems, volume 25 of Springer Series in Computational Mathematics. Springer-Verlag, Berlin, second edition, 2006. 
[33] U. Wilbrandt, C. Bartsch, N. Ahmed, N. Alia, Fe. Anker, L. Blank, A. Caiazzo, S. Ganesan, S. Giere, Gu. Matthies, R. Meesala, A. Shamim, J. Venkatesan, and V. John. ParMooN-A modernized program package based on mapped finite elements. Comput. Math. Appl., 74(1):74-88, 2017. 